1 Der Berliner Längsschnitt Medien: Rahmenbedingungen, Umsetzung, Stichprobenbeschreibung und Beschreibung der Skalen und Testinstrumente

Das von der Volkswagenstiftung geförderte Projekt Mediennutzung und Schulleistung des Kriminologischen Forschungsinstituts Niedersachsen (KFN) untersucht mithilfe eines interdisziplinären Forschungsansatzes die Auswirkungen, die ein verändertes Freizeit-, insbesondere ein verändertes Bildschirmmedienmenü von Kindern und Jugendlichen auf ihre Leistungsfähigkeit in Schule und Gesellschaft hat. Kern dieses Projekts ist der Berliner Längsschnitt Medien, eine medienpsychologische und medienpädagogische Längsschnittstudie. Um die genauen Wirkmechanismen von Mediennutzung besser interpretieren zu können, wird der Berliner Längsschnitt Medien von medienpsychologischen und neurophysiologischen Experimenten zum Einfluss der Medienrezeption auf die Konsolidierung von Wissensinhalten begleitet. Ziel des Berliner Längsschnitts Medien ist die umfassende Beantwortung der Frage, in welcher Weise sich die Mediennutzung auf Kinder und Jugendliche bezüglich ihrer Freizeitgestaltung, ihres Sozialverhaltens, ihrer körperlichen Entwicklung sowie ihrer Schulleistungen auswirkt. Außerdem soll geklärt werden, welche Veränderungen sich durch eine gezielte Intervention mit dem Ziel einer zeitlichen Reduktion der Mediennutzung erreichen lassen. In dem „lernenden Projekt" werden in Zusammenarbeit mit den teilnehmenden Schulen sinnvolle Konzepte für einen Medienunterricht entwickelt und getestet (Studiendesign: siehe Abbildung 1).

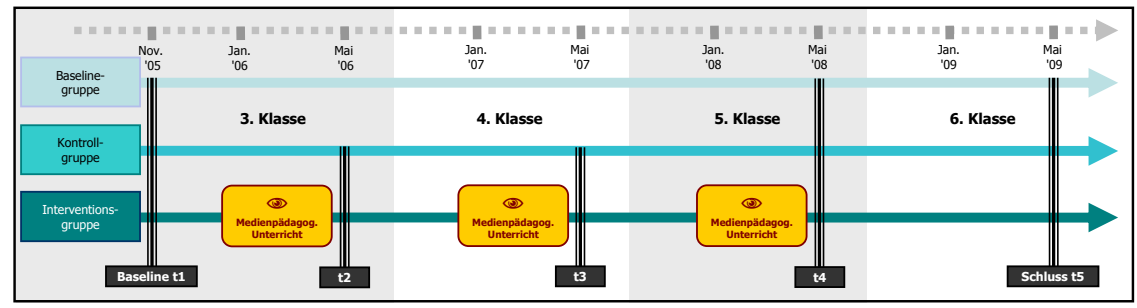

Abbildung 1. Studiendesign des Berliner Längsschnitt Medien.

Durch das Kontrollgruppendesign können zum einen bei der Evaluation des medienpädagogischen Unterrichts die für die Schulleistungen der Kinder wichtigen Variablen Intelligenz und Leistungsfähigkeit kontrolliert werden. Zum ande- 
ren können die Variablen familiäres Umfeld, Sozialverhalten und Freizeitverhalten als wichtige Aspekte bei der Aufklärung des Zusammenhangs von Mediennutzung und Schulleistungsentwicklung berücksichtigt werden. Durch den Einschluss einer Baselinegruppe können darüber hinaus mögliche Effekte, die auf die reine Befragung der Kinder und nicht auf den medienpädagogischen Unterricht zurückgehen, herausgefiltert werden.

\subsection{Stichprobenziehung}

Nach der Zustimmung zur Untersuchungsdurchführung durch die Berliner Senatsverwaltung für Bildung, Jugend und Sport im Mai 2005 wurden aus der Grundgesamtheit aller 1.042 Berliner ${ }^{1}$ Klassen der dritten Jahrgangsstufe (Stand

Tabelle 1. Stichprobenziehung und Teilnahmequoten.

\begin{tabular}{|c|c|c|c|c|}
\hline & Klassen & Lehrkräfte & Schüler/innen & Eltern \\
\hline Grundgesamtheit & 1.042 & - & 24.714 & - \\
\hline $\begin{array}{l}\text { Bereinigte Stichprobe } \\
(n>15)\end{array}$ & 1.009 & - & 24.352 & - \\
\hline Gezogene Stichprobe & 80 & - & 1.929 & - \\
\hline $\begin{array}{l}\text { t } 1 \text { (3. Klasse) } \\
\text { Untersuchungsstichprobe } \\
\text { Anzahl (Teilnahmequote) }\end{array}$ & $\begin{array}{c}47 \\
47(100 \%)\end{array}$ & $\begin{array}{c}47 \\
47(100 \%)\end{array}$ & $\begin{array}{c}1.129 \\
943(84 \%)\end{array}$ & $\begin{array}{c}943 \\
828(88 \%)\end{array}$ \\
\hline $\begin{array}{l}\text { t } 2 \text { (3. Klasse) } \\
\text { Untersuchungsstichprobe } \\
\text { Anzahl (Teilnahmequote) } \\
\text { Neue Schüler/innen (Quote) }\end{array}$ & $\begin{array}{c}40 \\
40(100 \%)\end{array}$ & $\begin{array}{c}40 \\
39(98 \%)\end{array}$ & $\begin{array}{c}967 \\
846(88 \%) \\
64(8 \%)\end{array}$ & $\begin{array}{l}- \\
- \\
-\end{array}$ \\
\hline $\begin{array}{l}\text { t } 3 \text { (4. Klasse) } \\
\text { Untersuchungsstichprobe } \\
\text { Anzahl (Teilnahmequote) } \\
\text { Neue Schüler/innen (Quote) }\end{array}$ & $\begin{array}{c}40 \\
40(100 \%)\end{array}$ & $\begin{array}{c}40 \\
39(98 \%)\end{array}$ & $\begin{array}{c}960 \\
835(87 \%) \\
52(6 \%)\end{array}$ & $\begin{array}{c}835 \\
686(82 \%)\end{array}$ \\
\hline $\begin{array}{l}\text { t } 4 \text { (5. Klasse) } \\
\text { Untersuchungsstichprobe } \\
\text { Anzahl (Teilnahmequote) } \\
\text { Neue Schüler/innen (Quote) }\end{array}$ & $\begin{array}{c}47 \\
44(94 \%)\end{array}$ & $\begin{array}{c}44 \\
40(91 \%)\end{array}$ & $\begin{array}{c}998 \\
827(83 \%) \\
98(12 \%)\end{array}$ & $\begin{array}{c}827 \\
594(72 \%)\end{array}$ \\
\hline $\begin{array}{l}\text { t } 5 \text { (6. Klasse) } \\
\text { Untersuchungsstichprobe } \\
\text { Anzahl (Teilnahmequote) } \\
\text { Neue Schüler/innen (Quote) }\end{array}$ & $\begin{array}{c}47 \\
44(94 \%)\end{array}$ & $\begin{array}{c}44 \\
41(93 \%)\end{array}$ & $\begin{array}{c}1.021 \\
806(79 \%) \\
50(6 \%)\end{array}$ & $\begin{array}{c}806 \\
571(71 \%)\end{array}$ \\
\hline
\end{tabular}

1 Das Bundesland Berlin wurde ausgewählt, da hier der Regelbesuch der Grundschule bis zur sechsten Klasse vorgesehen ist und Kinder - darüber hinaus mit gemischtem sozio-kulturellen Hintergrund - im selben Klassenkontext über einen Zeitraum von vier Jahren befragt werden können. 
Mai 2005: 24.714 Berliner Grundschüler und Grundschülerinnen) zunächst diejenigen Klassen mit einer Klassengröße $\mathrm{n}>15$ in die Stichprobenziehung aufgenommen. Es gingen somit insgesamt 24.352 Schüler und Schülerinnen in 1.009 Klassen in die Stichprobenziehung ein. 80 Grundschulklassen (jeweils unterschiedlicher Schulen) wurden per Zufall gezogen, von denen sich letztendlich 47 mit einer Gesamtzahl von 1.129 Schülerinnen und Schüler der dritten Klasse an der Längsschnittstudie beteiligten (vgl. Tabelle 1).

Die Schulen verteilen sich dabei repräsentativ auf die zwölf Stadtbezirke Berlins, wobei die Stadtbezirke im ehemaligen Ostteil der Stadt bereits bei der Stichprobenziehung leicht aufgewertet wurden (siehe Abbildung 2, Anhang A).

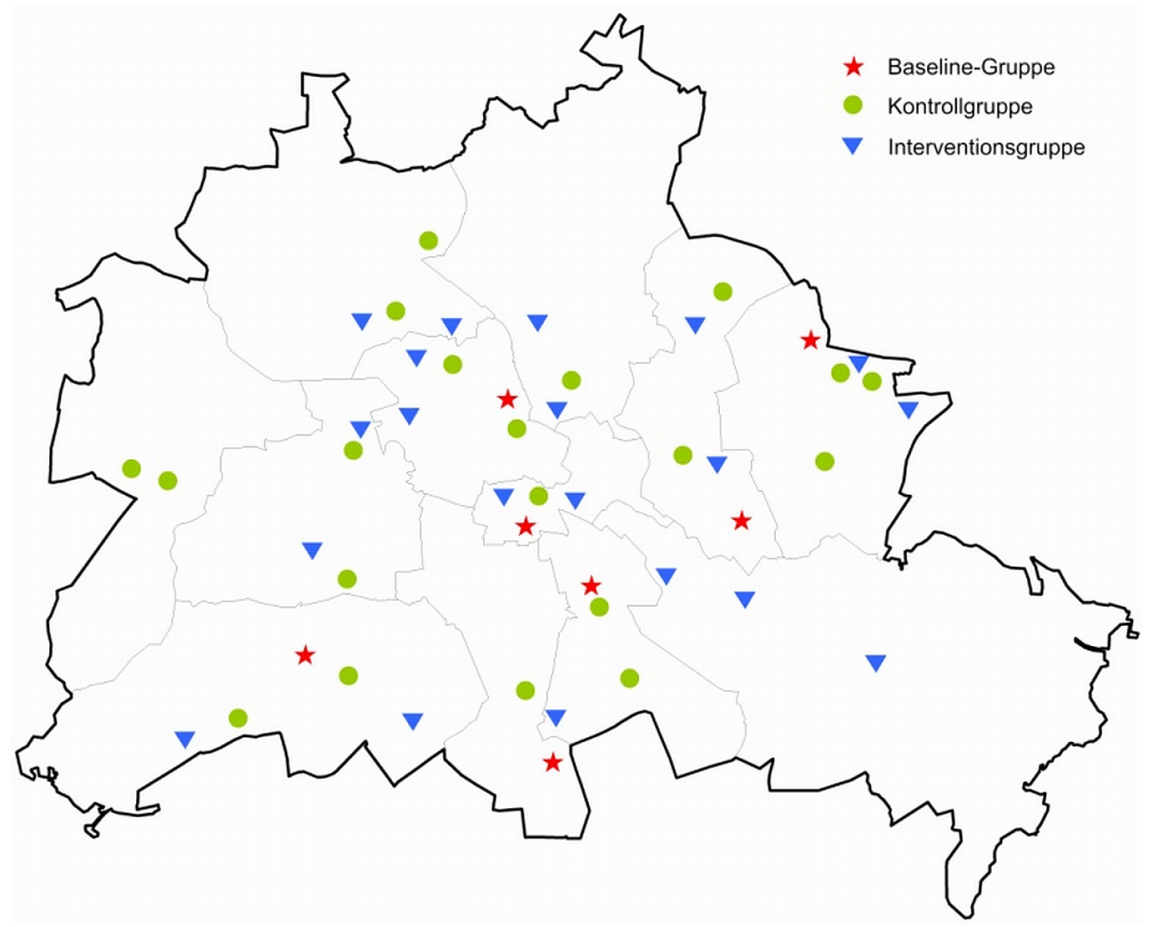

Abbildung 2. Verteilung der Schulen über das Stadtgebiet Berlin.

\subsection{Datenerhebungen}

Vor einer detaillierten Stichprobenbeschreibung soll zunächst die Durchführung der unterschiedlichen Messungen und Interventionseinheiten chronologisch ge- 
trennt nach Messung (Kapitel 1.2) und Intervention (Kapitel 1.3) beschrieben werden, um das Verständnis des Studiendesigns zu erleichtern.

\subsubsection{Basismessung $-\mathrm{t} 1$}

Im November 2005 fand (nach einem Pretest der Untersuchungsmaterialien und Untersuchungsdurchführung an zwei Hannoveraner Grundschulen) an den 47 teilnehmenden Berliner Grundschulen die erste Welle der Datenerhebung statt. Die Eltern der Grundschüler und Grundschülerinnen wurden vorab mit einem kurzen Elternbrief sowie einem Empfehlungsschreiben der Berliner Senatsverwaltung für Bildung, Jugend und Sport über die geplante Längsschnittuntersuchung informiert. Dieses Schreiben wurde für Eltern mit Migrationshintergrund ins Türkische, Arabische und Russische übersetzt. Als Rückmeldung auf das Informationsschreiben wurde die schriftliche Einverständniserklärung der Eltern eingeholt und nur diejenigen Kinder, deren schriftliche Erlaubnis der Eltern vorlag, durften an der Untersuchung teilnehmen. Es beteiligten sich zum Messzeitpunkt t 1 insgesamt 943 Schüler und Schülerinnen (84\% Teilnahmequote), 47 Lehrkräfte und 828 Eltern ( $88 \%$ Teilnahmequote) an der Befragung (siehe Tabelle 1).

Die Befragungen im Klassenverbund mittels standardisierter Fragebogeninstrumente (Übersicht siehe Tabelle 2, siehe Kapitel 1.5) fanden an zwei aufeinanderfolgenden Tagen während des Schulunterrichts statt und beanspruchten jeweils zwei Schulstunden. Die Durchführung der Befragung wurde von durch das KFN geschulten Interviewern und Interviewerinnen (Studierende der Psychologie) übernommen. Die Schulung fand ca. zwei Wochen vor der Befragung in den Räumen der Berliner Senatsverwaltung für Bildung, Jugend und Sport statt und beinhaltete eine ausführliche Besprechung der zur Verwendung kommenden Untersuchungsmaterialien sowie eine Einweisung in das Manual der Untersuchungsdurchführung.

Am ersten Untersuchungstag (durchschnittliche Befragungsdauer 1:58 Stunden) kam ein am KFN entwickelter 8-seitiger Fragebogen zu Mediennutzung, Medienausstattung, Freizeitverhalten, Soziodemografie und elterlichem Erziehungs- sowie Medienerziehungsverhalten zum Einsatz. Zusätzlich wurde der vierseitige Fragebogen zur Erfassung emotionaler und sozialer Schulerfahrungen von Grundschulkindern dritter und vierter Klassen (FEESS 3-4, Rauer \& Schuck, 2003) bearbeitet, der unter anderem das Selbstkonzept eigener Schulfähigkeiten und die erlebte soziale Integration erfasst. Die Kinder wurden während der gesamten Befragung von den Interviewern und Interviewerinnen anhand des eingesetzten Manuals durch diese Fragen geführt. Alle Fragen und Antwortmög- 
lichkeiten wurden durch den Interviewer oder die Interviewerin laut vorgelesen und gegebenenfalls erläutert, zusätzlich wurde die entsprechende Seite mittels eines Overheadprojektors an die Wand projiziert. Bei schwierigeren Fragen bekamen die Kinder darüber hinaus weitere mündliche Instruktionen durch den Interviewer oder die Interviewerin.

Am zweiten Testtag (durchschnittliche Testdauer 1:43 Stunden) wurden mit den Schülerinnen und Schülern drei verschiedene Leistungstests durchgeführt. Der Grundintelligenztest Skala 2 (CFT 20, 1. Teil) zur Bestimmung der allgemeinen Intelligenz, der Wortschatztest (WS) zur Bestimmung der verbalen Fähigkeiten und Fertigkeiten sowie der Zahlenfolgetest (ZF) zur Bestimmung der numerischen Fähigkeiten und Fertigkeiten (alle Weiß, 1998b). Wie der FEESS 3-4 wurden CFT 20, WS und ZF gemäß der im Test enthaltenen Manuale und Anweisungen für die Gruppentestung durchgeführt. An einem der beiden Untersuchungstage wurden darüber hinaus mit geeichten Messinstrumenten das Körpergewicht (in Hose und T-Shirt) sowie die Körpergröße zur Berechnung des BodyMass-Index (BMI) der Kinder bestimmt.

Zusätzlich zu den Angaben der Kinder füllte die Lehrkraft während des Klasseninterviews einen eigenen Fragebogen aus, in dem neben Angaben zur ausfüllenden Lehrkraft (Geschlecht, Alter, Funktion in der Klasse, unterrichtete Fächer) auch Angaben zur Medienausstattung an der Schule und deren Nutzungshäufigkeit gemacht werden sollten. Darüber hinaus wurden für jeden einzelnen Schüler oder jede einzelne Schülerin die Deutschkenntnisse bei der Einschulung sowie die Schulnoten in den Kernfächern Deutsch, Mathematik, Sachkunde sowie Sport notiert.

Neben dem Schüler- und Lehrerfragebogen kam auch ein Elternfragebogen zum Einsatz, der wie das Elterninformationsschreiben auch ins Russische, Arabische und Türkische übersetzt wurde, wobei die übersetzte Version des Fragebogens immer nur zusätzlich ausgegeben wurde. Im Elternfragebogen waren unter anderem Fragen zu Soziodemografie (z. B. Schulabschluss, Erwerbstätigkeit, Muttersprache, Familienstand), Mediennutzung (Eltern/Kind), zur Medienausstattung (Eltern/Kind), zum Medienerziehungsverhalten (getrennt für Fernsehen und Computer) sowie zur Einstellung gegenüber Medien und deren Nutzung enthalten. Darüber hinaus wurde das kindliche Sozialverhalten (Elternfragebogen über das Verhalten von Kindern und Jugendlichen - CBCL/4-18, Fragen I-VI; Arbeitsgruppe Deutsche Child Behavior Checklist, 1998), unter anderem in den Bereichen Sportarten, Hobbys, Vereinsmitgliedschaften und Pflichten zu Hause näher abgefragt.

Nach der ersten Messung wurde die Untersuchungsstichprobe unter Kontrolle der Mediennutzungsdaten sowie der Intelligenzdaten klassenweise zufällig den unterschiedlichen Untersuchungsbedingungen zugeordnet: in die Interventions- 
bedingung ( $\mathrm{n}=20$ Klassen, 498 potenzielle Schülerinnen und Schüler, 428 an der Befragung teilgenommene), die Kontrollbedingung, die keine Intervention erhielt ( $\mathrm{n}=20$ Klassen, 469 potenzielle Schülerinnen und Schüler, 402 an der Befragung teilgenommene), und einer Baselinegruppe, in der nur zu Beginn und zum vierten und fünften Messzeitpunkt der Studie gemessen wurde ( $\mathrm{n}=7$ Klassen, 162 potenzielle Schülerinnen und Schüler, 113 an der Befragung teilgenommene). Bei der Aufteilung in die unterschiedlichen Bedingungen wurde darauf geachtet, dass die den Bedingungen zugewiesenen Schulen räumlich voneinander getrennt sind. Diese räumliche Trennung wurde als notwendig erachtet, da die Interventionsmaßnahmen unter anderem auch das nähere Umfeld der Grundschulen mitgestalten sollen.

\subsubsection{Erste Kontrollmessung - t 2}

Im Mai/Juni 2006 fand an den 40 Treatment- und Kontrollschulen² die zweite Welle der Datenerhebung statt. Es beteiligten sich in den Treatment- und Kontrollklassen insgesamt 846 Schülerinnen und Schüler (88\% Teilnahmequote; $94 \%$ der Ausgangsstichprobe, 782 dieser Schülerinnen und Schüler hatten bereits zu t 1 teilgenommen) sowie 40 Lehrkräfte an der Befragung (siehe Tabelle 1). Die Durchführung wurde analog zum ersten Messzeitpunkt organisiert und umgesetzt. Im Unterschied zum ersten Messzeitpunkt wurde beim zweiten Messzeitpunkt jedoch keine Elternbefragung durchgeführt.

Aufgrund des Längsschnittdesigns der Studie unterscheiden sich die Untersuchungsmaterialien nur geringfügig zu t 1 . Unterschiede im Schülerfragebogen bestehen lediglich in kleinen Veränderungen und abwechselnden Schwerpunktsetzungen (siehe Tabelle 2). So entfielen die Fragen zur Soziodemografie, die zur Berechnung des Wohlstandsindexes benötigt wurden sowie die Fragen zur differenzierten Erfassung der Familienstruktur. Hinzu kamen Fragen zum Wohlbefinden in der letzten Woche. Alle anderen Untersuchungselemente (standardisierte Fragebögen und Messung der körperlichen Parameter) wurden auch in der zeitlichen Verteilung auf die Testtage analog zum ersten Messzeitpunkt durchgeführt (durchschnittliche Befragungsdauer 1:39 Stunden; durchschnittliche Testdauer 1:35 Stunden).

Der Lehrerfragebogen wurde in den Interventionsklassen zusätzlich um eine Rückmeldung zum Medienunterricht ergänzt.

2 Die Baselineklassen wurden erst wieder zum vierten Messzeitpunkt in die Befragung mit einbezogen. 


\subsubsection{Zweite Kontrollmessung - t 3}

Im Mai/Juni 2007 fand an den 40 Treatment- und Kontrollschulen die dritte Welle der Datenerhebung statt. Analog zum ersten Messzeitpunkt wurde beim dritten Messzeitpunkt wieder eine Elternbefragung durchgeführt. Es beteiligten sich an t 3 insgesamt 835 Schüler und Schülerinnen (87\% Teilnahmequote; $88 \%$ der Ausgangsstichprobe, 729 dieser Schülerinnen und Schüler hatten zu t 1 teilgenommen), 40 Lehrkräfte und 686 Eltern (82\% Teilnahmequote) an der Befragung (siehe Tabelle 1).

Die Untersuchungsmaterialien unterscheiden sich wiederum nur geringfügig von den vorherigen Messzeitpunkten. In Ergänzung zu den Fragen von t 2 wurde in der dritten Messung der Themenschwerpunkt Gewalt mit den Unterpunkten Schulgewalt (aus Opfer- und Täterperspektive), erlebte Elterngewalt, delinquenter Freundeskreis sowie delinquentes Verhalten in bestimmten Bereichen aus der Täterperspektive zum ersten Mal mit aufgenommen. Zusätzlich wurden die Persönlichkeitseigenschaften Empathie und Impulskontrolle (IVE, Inventar zur Erfassung von Impulsivität, Risikoverhalten und Empathie bei 9-bis 14-jährigen Kindern; Stadler, Janke \& Schmeck, 2004a) gemessen. Alle anderen Untersuchungselemente (standardisierte Fragebögen und Messung der körperlichen Parameter) wurden wiederum auch in der zeitlichen Verteilung auf die Testtage analog zu den ersten beiden Messzeitpunkten durchgeführt (durchschnittliche Befragungsdauer 1:43 Stunden; durchschnittliche Testdauer 1:31 Stunden).

Der eingesetzte Lehrerfragebogen war bis auf die Rückmeldung zum Medienunterricht in den Interventionsklassen identisch mit dem Lehrerfragebogen des t 2 .

Der Elternfragebogen wurde wie bei der ersten Befragung wiederum ins Russische, Arabische und Türkische übersetzt. Die Fragen zur Soziodemografie wurden um die Fragen zur Staatsangehörigkeit, Schulabschluss und Erwerbstätigkeit gekürzt.

\subsubsection{Dritte Kontrollmessung - t 4}

Im Mai/Juni 2008 fand an 44 der 47 Treatment-, Kontroll- und Baselineschulen die vierte Welle der Datenerhebung statt (2 Klassen der Baselinegruppe sowie eine Interventionsklasse beteiligten sich von diesem Messzeitpunkt an nicht mehr an der Befragung). Analog zum ersten und dritten Messzeitpunkt wurde beim vierten Messzeitpunkt eine Elternbefragung durchgeführt. Es beteiligten sich an t 4 insgesamt 827 Schüler und Schülerinnen (83\% Teilnahmequote; $69 \%$ der Ausgangsstichprobe, 648 dieser Schülerinnen und Schüler hatten zu t 1 teilge- 
nommen), 40 Lehrkräfte (91\% Teilnahmequote) und 594 Eltern (72\% Teilnahmequote) an der Befragung (siehe Tabelle 1).

In Ergänzung zu den Fragen von t 3 wurde in der vierten Messung der Themenschwerpunkt Gewalt um die Opferperspektive erlebter Gewalt außerhalb der Schule erweitert. Wieder mit aufgenommen wurden die Fragen zur Soziodemografie, die zur Berechnung des Wohlstandsindexes benötigt wurden sowie die Fragen zur differenzierten Erfassung der Familienstruktur. Als weiterer Schwerpunkt kam zu diesem Messzeitpunkt das Thema Computerspielabhängigkeit (KFN-CSAS-I; Mößle, Kleimann \& Rehbein, 2007) hinzu. Im Bereich der Mediennutzung wurde schließlich noch das Internet als weiteres Medium hinsichtlich elterlicher Erziehungskompetenz mit aufgenommen. Als zusätzliche Persönlichkeitseigenschaften wurden im Fragebogen der fünften Klasse schließlich „Hyperaktivität“, „Verhaltensprobleme mit Gleichaltrigen“ und ,prosoziales Verhalten" mithilfe des Strengths and Difficulties Questionnaire in der deutschen Übersetzung (SDQ-Deu; Goodman, 1997; Klasen et al., 2000) erhoben. Alle anderen Untersuchungselemente (standardisierte Fragebögen und Messung der körperlichen Parameter) wurden wiederum auch in der zeitlichen Verteilung auf die Testtage analog zu den ersten drei Messzeitpunkten durchgeführt, wobei der FEESS 3-4, der CFT 20, der WS sowie der ZF zum letzten Mal eingesetzt wurden (durchschnittliche Befragungsdauer 1:44 Stunden; durchschnittliche Testdauer 1:34 Stunden).

Der eingesetzte Lehrerfragebogen war bis auf die Rückmeldung zum Medienunterricht in den Interventionsklassen identisch mit dem Lehrerfragebogen des t 2 und t 3. Lediglich das Schulfach Sachkunde wurde durch Englisch ersetzt.

Der Elternfragebogen, wiederum ins Russische, Arabische und Türkische übersetzt, wurde in der fünften Klasse um die Fragen zum kindlichen Sozialverhalten (CBCL/4-18) gekürzt. Wieder aufgenommen wurden die Fragen zur Staatsangehörigkeit, Schulabschluss und Erwerbstätigkeit. Erweitert wurde der Elternfragebogen in der fünften Klasse, analog zum Fragebogen der Kinder, um die Medienerziehung im Bereich des Internets sowie um eine Frage zu den drei Lieblingscomputerspielen der Eltern.

\subsubsection{Schlussmessung $-\mathrm{t} 5$}

Im Mai/Juni 2009 fand an 44 der 47 Treatment-, Kontroll- und Baselineschulen die fünfte und letzte Welle der Datenerhebung vor dem bevorstehenden Schulwechsel statt. Analog zum ersten, dritten und vierten Messzeitpunkt wurde beim fünften Messzeitpunkt eine Elternbefragung durchgeführt. Es beteiligten sich an t 5 insgesamt 806 Schüler und Schülerinnen (79\% Teilnahmequote; $64 \%$ der 
Ausgangsstichprobe, 603 dieser Schülerinnen und Schüler hatten zu t 1 teilgenommen), 41 Lehrkräfte (93\% Teilnahmequote) und 571 Eltern (71\% Teilnahmequote) an der Befragung (siehe Tabelle 1).

Die Untersuchungsmaterialien unterscheiden sich wiederum nur geringfügig zu t 4. Unterschiede im Schülerfragebogen bestehen lediglich in zusätzlichen Fragen zur weiterführenden Schule. Darüber hinaus wurde Computerspielabhängigkeit mit dem überarbeiteten Instrument KFN-CSAS-II (Rehbein, Kleimann \& Mößle, 2009a) gemessen. Die durchschnittliche Befragungsdauer lag bei 1:37 Stunden.

Der eingesetzte Lehrerfragebogen wurde im Vergleich zu t 4 um die Rückmeldung zum Medienunterricht in den Interventionsklassen gekürzt und um eine Schullaufbahnempfehlung für jeden einzelnen Schüler bzw. jede einzelne Schülerin ergänzt.

Der Elternfragebogen, wiederum ins Russische, Arabische und Türkische übersetzt, wurde im Vergleich zu t 4 um allgemeine Fragen zur Einschätzung des Jugendmedienschutzes im Bereich der Computer- und Videospiele erweitert. Zusätzlich wurde die Frage zu den drei Lieblingsspielen auf beide Elternteile ausgedehnt.

In Tabelle 2 sind die unterschiedlichen Testinstrumente, die in den nachfolgenden Analysen zum Einsatz kamen, sowie die damit abgefragten Themengebiete noch einmal zusammenfassend dargestellt und zwar getrennt nach Schülersowie Eltern- und Lehrerfragebogen. Eine Beschreibung der eingesetzten Testverfahren (FEESS 3-4, Rauer \& Schuck, 2003; IVE, Stadler et al., 2004a; SDQDeu; Goodman, 1997; Klasen et al., 2000; CFT 20, ZF, WS, alle Weiß, 1998b) sowie der Skalen- und Itemkennwerte der jeweiligen Skalen des Schülerfragebogens finden sich in Kapitel 1.5. 
Tabelle 2. Übersicht über die eingesetzten Testinstrumente sowie über die erhobenen Variablen im Schüler-, Eltern- und Lehrerfragebogen.

\begin{tabular}{|c|c|c|c|c|c|c|}
\hline Instrument & Inhalt & $\mathrm{t} 1$ & $\mathrm{t} 2$ & t 3 & $\mathrm{t} 4$ & +5 \\
\hline \multirow{20}{*}{$\begin{array}{l}\text { Schülerfragebogen (Li- } \\
\text { teraturquellen der ein- } \\
\text { zelnen Skalen siehe } \\
\text { Kapitel 1.5.1) }\end{array}$} & Soziodemografie & $\mathrm{X}$ & & & $\mathrm{X}$ & $\mathrm{X}$ \\
\hline & Familienstruktur & $\mathrm{X}$ & $\mathrm{X}$ & $\mathrm{X}$ & $\mathrm{X}$ & $\mathrm{X}$ \\
\hline & Freizeitverhalten & $\mathrm{X}$ & $\mathrm{X}$ & $\mathrm{X}$ & $\mathrm{X}$ & $\mathrm{X}$ \\
\hline & Medienausstattung & $\mathrm{X}$ & $\mathrm{X}$ & $\mathrm{X}$ & $\mathrm{X}$ & $\mathrm{X}$ \\
\hline & Mediennutzungszeiten & $\mathrm{X}$ & & & $\mathrm{X}$ & $\mathrm{X}$ \\
\hline & Medieninhalte & $\mathrm{X}$ & $\mathrm{X}$ & $\mathrm{X}$ & $\mathrm{X}$ & $\mathrm{X}$ \\
\hline & $\begin{array}{l}\text { Wahrg. elterliches Erziehungs- } \\
\text { verhalten }\end{array}$ & $\mathrm{X}$ & $\mathrm{X}$ & $\mathrm{X}$ & $\mathrm{X}$ & $\mathrm{X}$ \\
\hline & $\begin{array}{l}\text { Wahrg. elterliches Medienerzie- } \\
\text { hungsverhalten }\end{array}$ & $\mathrm{X}$ & $\mathrm{X}$ & $\mathrm{X}$ & $\mathrm{X}$ & $\mathrm{X}$ \\
\hline & Erlebte Elterngewalt & & & $\mathrm{X}$ & $\mathrm{X}$ & $\mathrm{X}$ \\
\hline & Schulabsentismus & & & $\mathrm{X}$ & $\mathrm{X}$ & $\mathrm{X}$ \\
\hline & Delinquenz & & & $\mathrm{X}$ & $\mathrm{X}$ & $\mathrm{X}$ \\
\hline & Abweichendes Verhalten & & & $\mathrm{X}$ & $\mathrm{X}$ & $\mathrm{X}$ \\
\hline & Delinquenter Freundeskreis & & & $\mathrm{X}$ & $\mathrm{X}$ & $\mathrm{X}$ \\
\hline & Schulgewalt & & & $\mathrm{X}$ & $\mathrm{X}$ & $\mathrm{X}$ \\
\hline & Schulmobbing & & & $\mathrm{X}$ & $\mathrm{X}$ & $\mathrm{X}$ \\
\hline & Allgemeines Befinden & & $\mathrm{X}$ & $\mathrm{X}$ & $\mathrm{X}$ & $\mathrm{X}$ \\
\hline & $\begin{array}{l}\text { Computerspielabhängigkeit } \\
\text { (KFN-CSAS) }\end{array}$ & & & & $\mathrm{X}$ & $\mathrm{X}$ \\
\hline & Selbstkontrolle & & & & $\mathrm{X}$ & $\mathrm{X}$ \\
\hline & Schuleinstellung & & & & $\mathrm{X}$ & $\mathrm{X}$ \\
\hline & Weiterführende Schule & & & & & $\mathrm{X}$ \\
\hline \multirow{2}{*}{$\begin{array}{l}\text { FEESS 3-4 (Rauer \& } \\
\text { Schuck, 2003) }\end{array}$} & Erlebte eigene soziale Integration & $\mathrm{X}$ & $\mathrm{X}$ & $\mathrm{X}$ & $\mathrm{X}$ & $\mathrm{X}$ \\
\hline & Selbstkonzept der Schulfähigkeit & $\mathrm{X}$ & $\mathrm{X}$ & $\mathrm{X}$ & $\mathrm{X}$ & $\mathrm{X}$ \\
\hline \multirow{2}{*}{$\begin{array}{l}\text { IVE (Stadler et al., } \\
\text { 2004a) }\end{array}$} & Empathie & & & $\mathrm{X}$ & $\mathrm{X}$ & $\mathrm{X}$ \\
\hline & Impulsivität & & & $\mathrm{X}$ & $\mathrm{X}$ & $\mathrm{X}$ \\
\hline \multirow{3}{*}{$\begin{array}{l}\text { SDQ-Deu (Goodman, } \\
\text { 1997; Klasen et al., } \\
\text { 2000) }\end{array}$} & Hyperaktivität & & & & $\mathrm{X}$ & $\mathrm{X}$ \\
\hline & $\begin{array}{l}\text { Verhaltensprobleme mit Gleich- } \\
\text { altrigen }\end{array}$ & & & & $\mathrm{X}$ & $\mathrm{X}$ \\
\hline & Prosoziales Verhalten & & & & $\mathrm{X}$ & $\mathrm{X}$ \\
\hline
\end{tabular}




\begin{tabular}{|c|c|c|c|c|c|c|}
\hline Instrument & Inhalt & $\mathrm{t} 1$ & $\mathrm{t} 2$ & t 3 & $\mathrm{t} 4$ & t5 \\
\hline CFT-20 (Weiß, 1998b) & Intelligenz & $\mathrm{X}$ & $\bar{X}$ & $\mathrm{X}$ & $\mathrm{X}$ & \\
\hline WS (Weiß, 1998b) & $\begin{array}{l}\text { Allgemeinbildung und verbale } \\
\text { Verarbeitungskapazität }\end{array}$ & $\mathrm{X}$ & $\mathrm{X}$ & $\mathrm{X}$ & $\mathrm{X}$ & \\
\hline ZF (Weiß, 1998b) & $\begin{array}{l}\text { Numerische Verarbeitungskapa- } \\
\text { zität }\end{array}$ & $\mathrm{X}$ & $\mathrm{X}$ & $\mathrm{X}$ & $\mathrm{X}$ & \\
\hline $\begin{array}{l}\text { Waagen und Längen- } \\
\text { messgeräte }\end{array}$ & $\begin{array}{l}\text { Körpergewicht, Körpergröße, } \\
\text { (BMI) }\end{array}$ & $\mathrm{X}$ & $\mathrm{X}$ & $\mathrm{X}$ & $X$ & $\mathrm{X}$ \\
\hline \multirow{7}{*}{ Elternfragebogen } & Soziodemografie & $\mathrm{X}$ & & $\mathrm{X}$ & $\mathrm{X}$ & $\mathrm{X}$ \\
\hline & $\begin{array}{l}\text { Mediennutzung (Eltern und Kin- } \\
\text { der) }\end{array}$ & $\mathrm{X}$ & & $\mathrm{X}$ & $X$ & $\mathrm{X}$ \\
\hline & Freizeitverhalten (Kind) & $\mathrm{X}$ & & $\mathrm{X}$ & $\mathrm{X}$ & \\
\hline & $\begin{array}{l}\text { Medienausstattung (Eltern und } \\
\text { Kinder) }\end{array}$ & $X$ & & $\mathrm{X}$ & $\mathrm{X}$ & $\mathrm{X}$ \\
\hline & Medienerziehung & $X$ & & $\mathrm{X}$ & $\mathrm{X}$ & $\mathrm{X}$ \\
\hline & Jugendmedienschutz & & & & & $\mathrm{X}$ \\
\hline & Medienkenntnisse & & & & & $\mathrm{X}$ \\
\hline \multirow{4}{*}{ Lehrerfragebogen } & $\begin{array}{l}\text { Medienausstattung und -nutzung } \\
\text { in der Klasse }\end{array}$ & $\mathrm{X}$ & $\mathrm{X}$ & $\mathrm{X}$ & $\mathrm{X}$ & $\mathrm{X}$ \\
\hline & Schulnoten der Kinder & $\mathrm{X}$ & $\mathrm{X}$ & $\mathrm{X}$ & $\mathrm{X}$ & $\mathrm{X}$ \\
\hline & Rückmeldung Medienunterricht & & $\mathrm{X}$ & $\mathrm{X}$ & $X$ & \\
\hline & Schullaufbahnempfehlungen & & & & & $\mathrm{X}$ \\
\hline
\end{tabular}

\subsection{Medienpädagogischer Unterricht}

Die drei Blöcke mit medienpädagogischem Unterricht fanden jeweils zwischen den ersten vier Messzeitpunkten statt. Parallel zur ersten Datenerhebung wurde unter Berücksichtigung des Berliner Rahmenlehrplans für Medienerziehung in der Grundschule ein Unterrichtsprogramm erarbeitet, welches ab Februar 2006 in der Hälfte der Schulen ( $\mathrm{n}=20$ Klassen) zur Anwendung kam. Im Vorfeld der Entwicklung des Unterrichtsprogramms wurde im Sommer 2005 ein Workshop im KFN veranstaltet, bei dem Experten aus den Bereichen Medienpädagogik und Grundschulpädagogik sowie ein Produzent für Kinderlehrfilme teilnahmen. Auf dieser Grundlage entstand im Herbst 2005 ein Medienunterrichtskonzept für den ersten Interventionszeitpunkt (Frühjahr 2006). Das Konzept ermöglichte Lehrkräften in der Grundschule, im Kontext mehrerer Fächer (vor allem Deutsch und Sachkunde) einen geschlossenen Medienunterrichtsblock zu platzieren. Ein ers- 
ter Entwurf des Konzeptes wurde bereits im Rahmen der KFN-Schülerbefragung 2005 eingesetzt. In den zur Vorbereitung dieses Projektteils durchgeführten Lehrerworkshops wurde nochmals kritisches Feedback zur Unterrichtskonzeption eingeholt und in das Konzept eingearbeitet. Zur genauen Konzeption des Medienunterrichtsprogrammes siehe Kleimann (2011).

\subsubsection{Erste Interventionsphase - Vom Leichtmatrosen zum Medienlotsen}

Medienpädagogik findet in Deutschland zumeist in schulischen und außerschulischen Nischen statt. Eng verknüpft sind medienpädagogische Ansätze mit dem Begriff der Medienkompetenz. Medienkompetenz wird dabei als Ziel medienpädagogischen Handelns formuliert und kann in vier verschiedene Dimensionen eingeteilt werden: Mediengestaltung, Medienkritik, Medienkunde und Mediennutzung (vgl. Baacke, 1997). In der Vergangenheit fanden Mediengestaltung, Medienkritik und gerade in den letzten Jahren auch Medienkunde (hier vor allem die technische Kompetenz) vereinzelt in Curricula und Projektbeschreibungen Niederschlag, das Thema der alltäglichen Mediennutzung von Kindern und Jugendlichen wurde in der Schule jedoch kaum thematisiert. ${ }^{3}$

Ausgehend von den Ergebnissen bisheriger Forschung am KFN sowie den Resultaten der neueren Medienwirkungsforschung erschien bei der Entwicklung

3 Die Dimension der Mediengestaltung ist schon seit Jahrzehnten Unterrichtsinhalt. Immer da, wo Schüler eine eigene Schülerzeitung produzieren oder Artikel in Anlehnung an ,richtige" Zeitungen schreiben; immer da, wo Kinder mit Fotoapparat und Videokamera losgehen, um bestimmte Dinge ihrer Umwelt filmisch oder fotografisch festzuhalten; immer da, wo Schüler Collagen aus Zeitschriftenbildern zu bestimmten Themen erstellen sollen, setzten sie sich kreativ mit Medien und Medieninhalten auseinander und gestalten etwas Neues. Die Frage der Medienkritik wird häufig im Zusammenhang mit der Thematisierung von Werbung angesprochen. Schüler sprechen über die Besonderheiten von Werbung und über mögliche Manipulation durch Werbung. Ein beliebtes Thema im Deutschunterricht bei etwas älteren Kindern ist die Analyse von reißerischen Berichten in Boulevardmedien (z. B. Bildzeitung) im Vergleich zu ,seriösen“ Quellen. Die Dimension der Medienkunde umfasst das gesamte Wissen über die Funktionsweise von Medien und ihre definitorische Abgrenzung gegenüber anderen Medien. Besonders in der neuen Medienwelt ist medienkundlicher Unterricht für Lehrkräfte eine große Herausforderung, denn oftmals sind die zu behandelnden Inhalte auch für die Lehrkraft neu und herausfordernd. Am Beispiel des Einzugs des PCs in Klassenräume und der Verbreitung des Internets wurde dieses Thema in den letzten Jahren an nahezu allen Schulen innerhalb und außerhalb des normalen Unterrichts behandelt. Mediennutzung im Sinne exzessiver Mediennutzung wird im schulischen Alltag der Medienkompetenzvermittlung kaum thematisiert. Vor dem Hintergrund der schier unüberschaubaren Angebotsvielfalt, die bereits auf dem Medienmarkt für Kinder herrscht, verdient aber gerade dieser Bereich stärker in den Mittelpunkt medienpädagogischen Handelns gerückt zu werden. Mediennutzung nimmt bei vielen Kindern und Jugendlichen mehr Raum im Tag ein als Schule und Hausaufgaben zusammen. Umso wichtiger ist es, dass Kinder darüber nachdenken, welche Medieninhalte geeignet oder ungeeignet sind und welche Alternativen zur Mediennutzung existieren. 
neuer medienpädagogischer Konzepte besonders der Aspekt der alltäglichen Mediennutzung von Schülerinnen und Schülern relevant. Innerhalb des Projektes Berliner Längsschnitt Medien wurde aus diesem Grund eine Unterrichtseinheit für Kinder der dritten Klassen entwickelt, die besonders die Rolle von Medien im Alltag der Kinder beleuchtet. Dabei wurden die Lehrplanvorgaben des vom Berliner Landesinstitut für Schule und Medien (LISUM) entwickelten Zielgitters Medienerziehung in der Schule berücksichtigt. Dort heißt es:

- Die Schüler(innen) setzen sich mit medienbedingten Gefühlen auseinander.

- Die Schüler(innen) ermitteln, wie Mediennutzung ihren Tagesablauf beeinflusst.

- Die Schüler(innen) untersuchen kritisch solche Medien, die sie bewusst in ihrem Lebensalltag einsetzen.

Um eine Vermittlung derselben Inhalte in allen Klassen zu gewährleisten, wurde am KFN ein kleines Lesebuch mit dem Titel Vom Leichtmatrosen zum Medienlotsen entwickelt (die Materialien inklusive eines Leitfadens finden sich unter www.medienstudie.twoday.net). Die 15 aufeinander aufbauenden Kapitel dieses Lesebuches, die den Schullandheimausflug einer fiktiven Klasse zum Thema haben, verdeutlichen den Kindern kindgerecht verschiedene Aspekte von Mediennutzung und Medienwirkungen. So wird z. B. der Medienbegriff in der Geschichte eine Woche ohne Spongebob erarbeitet, die wissenschaftlich-empirische Methode, die Wichtigkeit genauer Messungen sowie die Bedeutung des Durchschnitts in der Geschichte Wie man hundert Millionen Würmer zählt oder die Medienwirkungen Sucht und soziale Isolation in der Geschichte Bettgeflüster. Der Aufbau der Geschichten erfolgte dabei nach folgenden Schritten:

- Medienumgang bewusst machen

- Folgen des Medienumgangs erkennen

- Alternativen entwickeln

- Umfeld sensibilisieren

Entlang dieser Dimensionen wurden in dem Lesebuch mit den drei Hauptelementen Medientagebuch, in das jedes Kind selbstständig für eine Woche sämtliche Aktivitäten inklusive der Dauer einzutragen hat, $\boldsymbol{T V}$-Programmplaner, einem Zweiwochenplan des Fernsehprogramms, und Medienvertrag drei medienpädagogische Instrumente vorgestellt, mit deren Hilfe die Lehrkräfte zu einem maßvollen Umgang mit Fernsehen und Computer- bzw. Videospielen anleiten sollten (vgl. Tabelle 3). Außerdem wurde ein Medienforscherpass entworfen, der an alle Kinder der Unterrichtsklassen vor Beginn des Medienunterrichtes verteilt wurde. In diesem Pass bekommen die Kinder für die Teilnahme an jeder Medienunterrichtseinheit und jeder Befragung einen Stempel der Klassenleitung, um das Engagement der Kinder während der verschiedenen Untersuchungs- und 
Unterrichtsphasen gleich bleibend hoch zu halten. Begleitet wurde der Medienunterricht durch Informationsveranstaltungen für Lehrkräfte und Eltern (Schritt: Umfeld sensibilisieren), die im Folgenden kurz beschrieben werden sollen.

Lehrerschulung. Um den 20 Lehrkräften in den Unterrichtsklassen alle notwendigen Kenntnisse zu vermitteln, die ihnen die Durchführung des Medienunterrichtes in ihren dritten Klassen ermöglichen sollten, wurden Mitte Februar 2006 drei vierstündige Fortbildungsworkshops durchgeführt, in denen die Lehrkräfte über die Anlage der Studie informiert wurden und ihnen einige Erkenntnisse aus der KFN-Schülerbefragung 2005 zum korrelativen Zusammenhang zwischen Mediennutzung und Schulleistungen von Grundschulkindern vermittelt wurden. Im Anschluss daran wurde ihnen das Unterrichtskonzept vorgestellt, wobei jede Lehrkraft eine Mappe mit allen notwendigen Unterrichtsmaterialien zur Verfügung gestellt bekam. Vier Lehrkräften, die an keinem der Workshoptermine teilnehmen konnten, wurden alle Materialien zusammen mit einem mehrseitigen Leitfaden zugesandt. Außerdem konnten die Lehrkräfte über eine eigens eingerichtete Internetseite (www.medienstudie.twoday.net) alle Materialien und einige Hintergrundinformationen herunterladen. Im Anschluss an die Workshops bekamen alle Lehrkräfte das Leseheft, passende Aufgabenblätter, die Medientagebücher und die Medienforscherpässe im Klassensatz zugesandt, um den organisatorischen und finanziellen Aufwand der jeweiligen Schulen zu minimieren.

Elternabend. Zeitgleich mit dem Beginn der Unterrichtseinheit wurde in den Unterrichtsklassen ein Informationsabend für die Eltern organisiert, der die anwesenden Erziehungsberechtigten für möglicherweise problematische Mediennutzungsmuster ihrer Kinder sensibilisieren und ihre eigene Mediennutzung bewusst machen sollte. Wie die Lehrkräfte wurden auch die Eltern mit Forschungsergebnissen aus der KFN-Schülerbefragung 2005 zum korrelativen Zusammenhang zwischen Mediennutzung und Schulleistungen von Grundschulkindern sowie weiterer internationaler Studien vertraut gemacht. Des Weiteren wurden den Eltern nützliche Hilfestellungen zur heimischen Mediennutzung vermittelt. Neben diesem etwa 45-minütigen Vortrag eines Mitglieds des KFN-Projektteams wurde der Klassenlehrerin oder dem Klassenlehrer auch eine Elterninformationsbroschüre Vom Leichtmatrosen zum Medienlotsen im Klassensatz zur Verfügung gestellt, wahlweise auf Deutsch, Türkisch oder Russisch. Diese Informationsbroschüre enthält neben Forschungsergebnissen auch Tipps zur Medienerziehung getrennt für Fernsehen, Computerspielen und Internetnutzung. Elternabende konnten in 17 Klassen realisiert werden, in zwei Klassen war ein Elternabend aus organisatorischen Gründen nicht möglich, eine Klasse beteiligte sich nicht an der Intervention. Im Durchschnitt besuchten 65 Prozent der Eltern die Elternabende. 
Tabelle 3. Aufbau, Themen und Lernstufen der Unterrichtseinheit Vom Leichtmatrosen zum Medienlotsen.

\begin{tabular}{|c|c|c|}
\hline Kapitel & Angesprochene Themen & Dimension \\
\hline $\begin{array}{l}\text { Eine Woche ohne } \\
\text { Spongebob }\end{array}$ & - Einführung des Medienbegriffes & $\begin{array}{l}\text { Medienumgang bewusst } \\
\text { machen }\end{array}$ \\
\hline $\begin{array}{l}\text { Alles Medien oder } \\
\text { was? }\end{array}$ & $\begin{array}{l}\text { Kategorisierung verschiedener } \\
\text { Medienarten } \\
-\quad \text { Vorstellung verschiedener Medi- } \\
\text { ennutzungsmodi }\end{array}$ & $\begin{array}{l}\text { Medienumgang bewusst } \\
\text { machen }\end{array}$ \\
\hline $\begin{array}{l}\text { Käpt'n Fischers Log-" } \\
\text { buch }\end{array}$ & $\begin{array}{l}\text { - Vorstellung der Tagebuchmetho- } \\
\text { de }\end{array}$ & $\begin{array}{l}\text { Medienumgang bewusst } \\
\text { machen }\end{array}$ \\
\hline Aus der Übung & $\begin{array}{l}\text { - Gründe für die Nutzung und Fas- } \\
\text { zination von Medien }\end{array}$ & $\begin{array}{l}\text { Medienumgang bewusst } \\
\text { machen }\end{array}$ \\
\hline Das Freizeitlogbuch & $\begin{array}{l}\text { - Aufgabenbeschreibung Freizeit- } \\
\text { und } \\
\text { - } \text { Medientagebuch }\end{array}$ & $\begin{array}{l}\text { Medienumgang bewusst } \\
\text { machen }\end{array}$ \\
\hline $\begin{array}{l}\text { Wie man hundert Mil- } \\
\text { lionen Würmer zählt }\end{array}$ & $\begin{array}{ll}\text { - } & \text { Erklärung der wissenschaftlich- } \\
\text { empirischen Methodik } \\
\text { - } \quad \text { Wichtigkeit genauer Messungen } \\
\text { - }\end{array}$ & $\begin{array}{l}\text { Medienumgang bewusst } \\
\text { machen }\end{array}$ \\
\hline $\begin{array}{l}\text { Wie man richtig aus- } \\
\text { wertet }\end{array}$ & $\begin{array}{l}\text { Einführung in die systematische } \\
\text { Auswertung der Tagebuchanga- } \\
\text { ben } \\
-\quad \text { Vergleich eigenen Medienverhal- } \\
\text { tens mit dem Medienverhalten } \\
\text { anderer Kinder }\end{array}$ & $\begin{array}{l}\text { Medienumgang bewusst } \\
\text { machen }\end{array}$ \\
\hline $\begin{array}{l}\text { Den ganzen Tag vor } \\
\text { der Spielkonsole }\end{array}$ & $\begin{array}{l}\text { - Unterscheidung Spielkonsole, } \\
\text { Computer } \\
\text { - } \text { Beschreibung eigener technischer } \\
\text { Medienkompetenz im Vergleich } \\
\text { zu den Eltern } \\
\text { - Beschreibung eigener Gefühle } \\
\text { nach stundenlangem Medienkon- } \\
\text { sum } \\
\text { - Vor- und Nachteile von Geräten } \\
\text { im Zimmer }\end{array}$ & $\begin{array}{l}\text { Medienumgang bewusst } \\
\text { machen } \\
\text { Folgen des Medienum- } \\
\text { gangs erkennen }\end{array}$ \\
\hline Ganz schön schlimm & 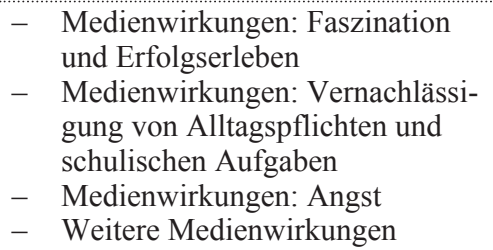 & $\begin{array}{l}\text { Folgen des Medienum- } \\
\text { gangs erkennen }\end{array}$ \\
\hline
\end{tabular}


- Medienwirkungen: Abhängigkeit

Bettgeflüster $\quad-\quad$ Medienwirkungen: Soziale Isolation

Folgen des Medienumgangs erkennen

- Wahrgenommene Mediennutzung von Erwachsenen (Eltern,

Ein schlechtes Vorbild Lehrer/innen)

Folgen des Medienumgangs erkennen

- Diskussionsangebot: Der richtige Alternativen entwickeln Umgang mit Fernsehen

- Gründe für die Fernsehnutzung:

Leuchttürme und Lotsen

Langeweile, Gewohnheit

- Möglichkeiten und Grenzen der

Alternativen entwickeln Selbstkontrolle

- Einführung des Fernsehplans als Methode der Selbstregulierung

Der Zweiwochenplan - Einführung der Aufgabe, beliebte nicht-mediale Freizeitbeschäfti-

Alternativen entwickeln gungen $\mathrm{zu}$ finden und zu beschreiben

- Unterscheidung virtueller Misserfolge und als real wahrgenomme-

Brettspiele und Lerncomputer ner Misserfolg in Spielsituationen

- sinnvoller Einsatz von Medien

- Beschreibung non-medialer Freizeitmöglichkeiten

- Einführung des Medienvertrages

Der Vertrag
- Mögliche Dimensionen eines Medienvertrages
Alternativen entwickeln

Alternativen entwickeln

\subsubsection{Zweite Interventionsphase - Die ELIZA-Protokolle}

Die Medienunterrichtseinheiten, die innerhalb unserer Modellstudie zum Einsatz kommen, sollen es Kindern ermöglichen, ihre eigene Freizeitmediennutzung zu analysieren, zu reflektieren und auch kritisch zu hinterfragen. Wichtig ist dabei, dass Schülerinnen und Schüler stets den Eindruck haben, dass der Teil ihrer Freizeit, der von Mediennutzung bestimmt wird, nicht einfach verurteilt wird, sondern dass immer beide Seiten berücksichtigt werden: einerseits die Faszinationskraft, die besonders von neuen, interaktiven Medienformaten ausgeht, andererseits aber auch die Risiken, die mit der Nutzung elektronischer Medien verbunden sein können. Dabei wurde während der Konzeption immer der Dreischritt „Sensibilisierung“, „Problematisierung“ und „Verhaltensanpassung“ verfolgt. In der ersten Unterrichtseinheit stand die „Sensibilisierung“ sehr klar im Vordergrund. So wurden Lehrkräfte sowie Schülerinnen und Schüler auf eine eher spie- 
lerische Art für das Thema der Mediennutzung im Alltag sensibilisiert (siehe oben).

In der zweiten Unterrichtseinheit sollte der zweite Punkt, die Problematisierung bestimmter Mediennutzungsmuster, stärker betont werden. In der Geschichte mit dem Titel Die ELIZA-Protokolle stehen explizit problematische Mediennutzungsmuster von Kindern und Jugendlichen im Vordergrund (die Materialien inklusive eines Leitfadens finden sich unter www.medienstudie.twoday.net). In den einzelnen Kapiteln der Geschichte werden abhängiges Computerspielen, die Nutzung von Gewaltmedieninhalten, die Beschränktheit computervermittelter Interaktion, aber auch medial vermittelte Schönheitsideale thematisiert. Die einzelnen Kapitel sind dabei um das Computerprogramm ELIZA angesiedelt, welches 1966 von Joseph Weizenbaum entwickelt wurde, um zu zeigen, wie mittels eines Computerprogramms ein menschlicher Gesprächspartner simuliert werden kann. Das Computerprogramm formuliert hierzu die Aussagen des Gesprächspartners in Fragen um. So beinhalten die einzelnen Kapitel neben einer Einführung in das Computerprogramm ELIZA (ein Gespräch in der Dunkelheit) auch Protokolle von Gesprächen mit ELIZA über z. B. Handys, SMS, Chatten, Castingshows sowie über den Fluch nicht dem klassischen Schönheitsideal zu entsprechen (Fio$n a$ ) oder über das Spielen von Gewaltcomputerspielen (Mario). Generell geht es in Die ELIZA-Protokolle um die Abhängigkeit von Medienformaten wie dem Computerspiel, aber auch dem Fernsehen oder dem Chatroom. Es werden auffällige Formen der Mediennutzung dargestellt und in ihren Auswirkungen beschrieben. Dabei wird gezeigt, dass Medien für Kinder und Jugendliche oftmals unverzichtbar sind, und sowohl als Kommunikationsmittel wie auch in ihrer Rolle als Unterhaltungsmedium einen zentralen Platz in ihrem Leben einnehmen. Den Kindern soll vermittelt werden, dass Medien manchmal auch nur Ersatz für andere Bedürfnisse, Ablenkung in kleineren oder größeren Lebenskrisen sein können oder große Teile anderer, wertvoller Freizeitaktivitäten verdrängen können. Dies zu erkennen und in jedem der in der Geschichte erzählten Fälle zu entscheiden, wo Medien helfen und wo sie schaden können, ist Hauptanliegen der zweiten Unterrichtseinheit.

Zur Umsetzung wurden wie bereits im Vorjahr innerhalb eintägiger Lehrerworkshops die Inhalte eines Lesebüchleins für die teilnehmenden Schülerinnen und Schüler entwickelt. Das Lesebüchlein (vgl. Tabelle 4) wurde wie bei der ersten Einheit zusammen mit Bearbeitungsvorschlägen allen teilnehmenden Kindern und Lehrkräften vom KFN zur Verfügung gestellt.

Zusätzlich wurde über die Klassenlehrer und Klassenlehrerinnen allen Eltern der Interventionsklassen eine DVD mit einem Fernsehbeitrag in der Sendung nano (3Sat) vom 10. 09. 2007 übergeben, der die Ziele, Inhalte und erste Ergebnisse des Projektes Mediennutzung und Schulleistung zum Inhalt hatte. 
Tabelle 4. Aufbau, Inhalt und Themen der Unterrichtseinheit „Die Eliza-Protokolle“.

\begin{tabular}{|c|c|c|}
\hline Kapitel & Inhalt & Angesprochene Themen \\
\hline 1. Nachtschicht & Einführung der Hauptfigur Bela & $\begin{array}{ll}- & \text { Sorgen und Probleme im Zusam- } \\
\text { menleben der Familie } \\
- & \text { Einsamkeit } \\
- & \text { Schulprobleme } \\
- & \text { Verwenden von Internetseiten } \\
& \text { wie wikipedia.de für die Haus- } \\
& \text { aufgaben }\end{array}$ \\
\hline $\begin{array}{l}\text { 2. Ein Ge- } \\
\text { spräch in der } \\
\text { Dunkelheit }\end{array}$ & $\begin{array}{l}\text { Einführung des Computerpro- } \\
\text { gramms ELIZA }\end{array}$ & $\begin{array}{l}\text { - } \begin{array}{l}\text { Faszination und Grenzen künstli- } \\
\text { cher Intelligenz }\end{array} \\
-\quad \text { Faszination von Computerspielen }\end{array}$ \\
\hline $\begin{array}{l}\text { 3. Ich bin ein } \\
\text { Meister aller } \\
\text { Waffen }\end{array}$ & $\begin{array}{l}\text { Belas Gespräch mit ELIZA über } \\
\text { seine Sorgen und die Faszinations- } \\
\text { kraft von Computerspielen }\end{array}$ & $\begin{array}{ll}- & \text { Konflikte im Freundeskreis } \\
- & \text { Computerspielsucht } \\
- & \text { Einsamkeit }\end{array}$ \\
\hline 4. Fiona & $\begin{array}{l}\text { Protokoll eines Gesprächs zwi- } \\
\text { schen ELIZA und einem Mädchen } \\
\text { namens Fiona über Handys, SMS, } \\
\text { Chatten, Casting-Shows und über } \\
\text { den Fluch, nicht dem klassischen } \\
\text { Schönheitsideal zu entsprechen }\end{array}$ & $\begin{array}{l}-\quad \text { Umzug in eine andere Stadt } \\
\text { (Neue Klasse, Verlust der alten } \\
\text { Freunde) } \\
-\quad \text { Neue Medien als Instrumente der } \\
\text { Kommunikation mit alten Freun- } \\
\text { den } \\
-\quad \text { Konflikte mit Eltern über die Me- } \\
\text { diennutzung (Zeit, Kosten) } \\
-\quad \text { Probleme mit dem in den Medien } \\
\text { vermittelten Schönheitsideal }\end{array}$ \\
\hline 5. Mario & $\begin{array}{l}\text { Protokoll eines Gesprächs zwi- } \\
\text { schen ELIZA und Mario über das } \\
\text { Spielen von Gewaltcomputerspie- } \\
\text { len }\end{array}$ & $\begin{array}{l}\text { - Negative Wirkungen von Gewalt } \\
\text { in Computerspielen } \\
\text { - } \text { Gründe der Faszination von Ge- } \\
\text { walt in den Medien } \\
-\quad \text { Umgang mit vertraulichen Infor- } \\
\text { mationen }\end{array}$ \\
\hline 6. Notbremse & $\begin{array}{l}\text { ELIZA wird größenwahnsinnig } \\
\text { und dringt in Belas Computer ein }\end{array}$ & $\begin{array}{ll}- & \text { Konsequenzen aus bedenklichem } \\
\text { Mediennutzungsverhalten } \\
- & \text { Die Rolle der Eltern in der Medi- } \\
\text { enerziehung } \\
-\quad \text { Denkstrukturen von Computern - } \\
\text { Denkstrukturen von Menschen } \\
-\quad \text { Sicherheit im Internet und Schutz } \\
\text { vor unerwünschten Übergriffen }\end{array}$ \\
\hline 7. Abschied & $\begin{array}{l}\text { Belas letztes Gespräch mit ELIZA. } \\
\text { Es wird deutlich, dass Kapitel } 3 \\
\text { bis } 6 \text { von Bela nur geträumt wur- } \\
\text { den }\end{array}$ & - Auflösung der Geschichte \\
\hline
\end{tabular}




\subsubsection{Dritte Interventionsphase - Medienlotsen gesucht!}

Im Februar 2008 wurde im Rahmen von drei Workshops zusammen mit den Lehrerinnen und Lehrern der Unterrichtsklassen das Unterrichtsprogramm für die dritte Unterrichtseinheit abgestimmt. Konzeptioneller Kern dieser Unterrichtseinheit war die Entwicklung (medien-)freizeitbezogener Alternativen, die der Forderung nach einer vielfältigen und anregungsreichen Freizeitgestaltung für Kinder nachkommen. Die Nutzung von elektronischen Medien sollte dabei die Aufgabe einer Bereicherung des Verhaltensspielraums der Kinder haben, nicht aber zu einer Verarmung der Freizeit durch eine zu starke Medienfokussierung führen.

Als wichtigstes Ergebnis dieser Lehrkräfteworkshops kristallisierte sich der Wunsch heraus, dass dieser Reflexionsprozess über eine vielfältige, anregende Freizeitgestaltung mit dem produktiven Einsatz elektronischer Medien kombiniert werden soll, um auch durch die Unterrichtsmethode selbst den Fokus auf eine produktive, kreative Mediennutzung zu lenken. Um hier den sehr unterschiedlichen zeitlichen und technischen Möglichkeiten aller Klassen gerecht zu werden, wurde den Klassen kein fertiges Unterrichtspaket zur Verfügung gestellt, sondern ein Wettbewerb unter dem Titel Medienlotsen gesucht! ausgeschrieben, in dem jede Klasse unter Verwendung des Materials der ersten beiden Unterrichtseinheiten ihre bereits erlernten Kompetenzen im Umgang mit elektronischen (Freizeit-)Medien zeigen und einem breiteren Publikum zugänglich machen sollte.

Noch stärker als bei den Unterrichtsworkshops der letzten Jahre offenbarten sich allerdings die zahlreichen Probleme, die insbesondere mit Lehrkraftwechseln in den Unterrichtsklassen verknüpft sind. So zeigten sich mit Verweis auf die Belastung durch den Lehrplan und andere Klassenaktivitäten zunächst längst nicht alle neuen Lehrerinnen und Lehrer bereit, eine weitere Unterrichtseinheit durchzuführen. Zudem waren einige der Teilnehmer durch ihre Vorgänger in der Klasse nicht hinreichend über das Projekt und seine Hintergründe informiert worden, obwohl nachdrücklich darum gebeten wurde. Vor diesem Hintergrund haben sich leider lediglich sechs Klassen beteiligt und fünf Beiträge sind durch Unterrichtsklassen eingereicht worden.

In Tabelle 5 sind die unterschiedlichen Elemente der drei medienpädagogischen Unterrichtseinheiten noch einmal zusammenfassend dargestellt. 
Tabelle 5. Elemente der medienpädagogischen Unterrichtseinheiten.

\begin{tabular}{|c|c|c|c|}
\hline Zielgruppe & $\begin{array}{l}\text { Vom Leichtmatrosen } \\
\text { zum Medienlotsen } \\
\text { (März-April 2006) }\end{array}$ & $\begin{array}{l}\text { Die ELIZA-Protokolle } \\
\text { (März-April 2007) }\end{array}$ & $\begin{array}{l}\text { Medienlotsen gesucht! } \\
\text { (März - April 2008) }\end{array}$ \\
\hline \multirow[t]{2}{*}{ Lehrkräfte } & Workshop & Workshop & Workshop \\
\hline & Unterrichtsmaterial & Unterrichtsmaterial & Ideen zur Umsetzung \\
\hline \multirow[t]{2}{*}{ Eltern } & Informationsabend & DVD & \\
\hline & Informationsbroschüre & & \\
\hline \multirow[t]{6}{*}{ Schüler/innen } & Lesebuch & Lesebuch & offener Wettbewerb \\
\hline & Fragen und Aufgaben & Fragen und Aufgaben & Medienforscherpass \\
\hline & Medientagebuch & Medienforscherpass & \\
\hline & TV-Programmplaner & & \\
\hline & Medienvertrag & & \\
\hline & Medienforscherpass & & \\
\hline
\end{tabular}

\subsection{Stichprobenbeschreibung}

Insgesamt beteiligten sich 1.207 Berliner Grundschülerinnen und -schüler, 1.085 Eltern sowie 89 Lehrkräfte an mindestens einem der fünf Messzeitpunkte an der Befragung, wobei die gemachten Angaben aufgrund der randomisierten Stichprobenziehung für das Bundesland Berlin als repräsentativ erachtet werden können. Im Folgenden soll die Stichprobe getrennt nach Schülerinnen und Schülern, Eltern und Lehrkräften in ihrer Beteiligung an den unterschiedlichen Messzeitpunkten näher beschrieben werden.

\subsubsection{Schülerinnen und Schüler}

Untersuchungsteilnahme. Tabelle 1 (Stichprobenziehung) war bereits zu entnehmen, dass zu den unterschiedlichen Messzeitpunkten neue Schüler und Schülerinnen zur Stichprobe hinzukamen (t 2: 64; t 3: 52; t 4: 98; t 4: 50), sodass die Stichprobe über den Befragungszeitraum erfreulicherweise von 943 Schülerinnen und Schülern zu t 1 auf 1.207 Schülerinnen und Schüler zu t 5, die sich an einem der fünf Messzeitpunkte an der Befragung beteiligten, angewachsen ist. Die größte Zunahme während der vierten Befragungswelle ist mit dem nach der vierten Klasse (t 3) möglichen Wechsel der Schülerinnen und Schüler auf eine weiterführende Schule sowie einer damit einhergehenden Neustrukturierung der Klassen verbunden. Diesem positiven Effekt eines Stichprobenwachstums von 
264 Schülerinnen und Schülern steht allerdings eine Stichprobenmortalität von 401 Schülerinnen und Schülern, insbesondere wiederum nach der vierten Klasse (176 Schülerinnen und Schüler), gegenüber. Auf die Ausgangsstichprobe zu t 1 von 943 Schülerinnen und Schülern bezogen, haben 340 Schülerinnen und Schüler (36\%) zu t 5 nicht mehr an der Befragung teilgenommen. 61 Schülerinnen und Schüler sind nach dem ersten Messzeitpunkt zur Stichprobe hinzugekommen, aber auch vor dem fünften Messzeitpunkt wieder aus der Stichprobe ausgeschieden.

Aus diesem Grund wurde erstmals zum vierten Messzeitpunkt im Lehrerfragebogen detailliert für jedes Kind, welches jemals an der Befragung teilgenommen hat, der Grund des Weggangs erhoben vgl. Tabelle 6). Nach Auskunft der Lehrkräfte ( 4 und t 5) besuchten zum Zeitpunkt der fünften Befragungswelle noch $832(69 \%)$ der insgesamt 1.207 Kinder jeweils die untersuchte Klasse. Während oder nach der dritten Klasse sind 43 Kinder (4\%) umgezogen, ein Kind musste die Klasse wiederholen $(0.08 \%)$. Nach der vierten Klasse sind 66 Schülerinnen und Schüler ( $5 \%$ ) umgezogen, drei Kinder $(0.25 \%)$ haben die Klasse wiederholt. Weitere 96 Kinder (8\%) wechselten nach der vierten Klasse auf eine weiterführende Schule. Nach der fünften Klasse sind 18 Kinder (1\%) aus der Untersuchungsstichprobe ausgeschieden weil sie umgezogen sind, zwei Kinder mussten die Klasse wiederholen $(0.17 \%)$. Weitere 4 Kinder $(0.33 \%)$ wechselten nach der fünften Klasse auf eine weiterführende Schule. Schließlich sind drei Kinder $(0.25 \%)$ nicht mehr in der Untersuchungsstichprobe, weil sie die Klasse innerhalb der Schule wechselten und sechs Kinder $(0.5 \%)$ wünschten keine weitere Teilnahme an der Untersuchung. Zu insgesamt 134 Schülerinnen und Schülern (11\%, wovon 41 zu t 4 noch die Schulklasse besuchten) liegen schließlich keine Angaben zu t 5 vor, da entweder zu diesem Kind keine Angaben gemacht wurden $(65 ; 5 \%)$, der Lehrerfragebogen insgesamt nicht ausgefüllt wurde (12; $1 \%$ ) oder die komplette Klasse nicht an t 4 oder t 5 teilnahm (57; 5\%).

Betrachtet man die Stichprobe getrennt nach Untersuchungsgruppe (siehe Tabelle 6), fällt zunächst auf, dass in der Baselinegruppe mit 8.0 Prozent im Vergleich zu beiden anderen Untersuchungsgruppen, die miteinander vergleichbar sind (Interventionsgruppe: 19.0\%, Kontrollgruppe: $21.5 \%$ ), insgesamt deutlich weniger Kinder aus der Stichprobe ausgeschieden sind. Differenziert man zusätzlich nach dem Grund (Umzug, Wechsel) muss festgehalten werden, dass in der Kontrollgruppe im Vergleich zur Interventions- und Baselinegruppe deutlich mehr Kinder aufgrund eines Umzuges nicht mehr an der Untersuchung teilgenommen haben. Hinsichtlich des Wechsels auf eine weiterführende Schule sind Kontroll- und Interventionsgruppe wieder annähernd vergleichbar, lediglich die Baselinegruppe fällt deutlich nach unten ab. Das Stichprobenwachstum ist in den drei Untersuchungsgruppen annähernd vergleichbar. 
Tabelle 6. Teilnahmestatus nach Untersuchungsgruppe.

\begin{tabular}{|c|c|c|c|c|c|c|}
\hline & \multirow[b]{2}{*}{$\begin{array}{l}\text { insge- } \\
\text { samt }\end{array}$} & \multicolumn{4}{|c|}{ Aus der Stichprobe ausgeschieden* } & \multirow{2}{*}{$\begin{array}{l}\text { Neu in } \\
\text { der } \\
\text { Stich- } \\
\text { probe }\end{array}$} \\
\hline & & $\begin{array}{l}\text { Umgezo- } \\
\text { gen }\end{array}$ & Wechsel $^{4}$ & sonstige & $\begin{array}{l}\text { fehlende } \\
\text { Angabe }\end{array}$ & \\
\hline Baselinegruppe & 141 & $\begin{array}{c}8 \\
(5.7 \%)\end{array}$ & $\begin{array}{c}4 \\
(2.8 \%)\end{array}$ & - & $\begin{array}{c}35 \\
(24.8 \%)\end{array}$ & $\begin{array}{c}28 \\
(19.9 \%)\end{array}$ \\
\hline $\begin{array}{l}\text { Interventions- } \\
\text { gruppe }\end{array}$ & 554 & $\begin{array}{c}52 \\
(9.4 \%)\end{array}$ & $\begin{array}{c}53 \\
(9.6 \%)\end{array}$ & $\begin{array}{c}8 \\
(1.4 \%)\end{array}$ & $\begin{array}{c}62 \\
(11.9 \%)\end{array}$ & $\begin{array}{c}126 \\
(22.7 \%)\end{array}$ \\
\hline Kontrollgruppe & 512 & $\begin{array}{c}67 \\
(13.1 \%)\end{array}$ & $\begin{array}{c}43 \\
(8.4 \%)\end{array}$ & $\begin{array}{c}7 \\
(1.4 \%)\end{array}$ & $\begin{array}{c}37 \\
(7.2 \%)\end{array}$ & $\begin{array}{c}110 \\
(21.5 \%)\end{array}$ \\
\hline Gesamt & 1.207 & $\begin{array}{c}127 \\
(10.5 \%)\end{array}$ & $\begin{array}{c}100 \\
(8.3 \%)\end{array}$ & $\begin{array}{c}15 \\
(1.2 \%)\end{array}$ & $\begin{array}{c}134 \\
(11.1 \%)\end{array}$ & $\begin{array}{c}264 \\
(21.8 \%)\end{array}$ \\
\hline
\end{tabular}

Anmerkung. Umgezogen $=$ Umgezogen nach der 3., 4. oder 5. Klasse. Wechsel $=$ Wechsel auf eine weiterführende Schule nach der 4. oder 5. Klasse. Neu in der Stichprobe = Zur Stichprobe hinzugekommen nach der 4. oder 5. Klasse. * Nur Kinder zu denen Angaben aus dem Lehrerfragebogen vorliegen.

Die 1.207 Kinder verteilen sich dabei wie folgt auf die unterschiedlichen Messzeitpunkte (siehe Tabelle 7). 495 Schülerinnen und Schüler (41\%) beteiligten sich an allen fünf Befragungswellen, $122(10 \%)$ an vier Befragungswellen, 270 (22\%) an drei Befragungswellen, $165(14 \%)$ an zwei Befragungswellen sowie $155(13 \%)$ an nur einer Befragungswelle. Die Teilnahmequoten unterscheiden sich dabei zwischen den Gruppen nur geringfügig. So haben in der Baseline- und in der Interventionsgruppe jeweils 45 Prozent der Schülerinnen und Schüler an allen ihnen möglichen Messzeitpunkten teilgenommen. Die bessere Beteiligung in der Kontrollgruppe (48\%) kann unter anderem auch dadurch erklärt werden, dass in der Baselinegruppe zwei komplette Klassen und in der Interventionsgruppe eine komplette Klasse bei den letzten beiden Messzeitpunkten ( 4 und t 5) wegfiel, da dort keine Befragung mehr realisiert werden konnte. Insgesamt liegen somit zu 559 Schülerinnen und Schülern (46\%) hinsichtlich der Messzeitpunkte komplette Datensätze vor. Für 887 (73\%) Kinder sind entweder komplette Datensätze oder in der Interventions- und Kontrollgruppe zu mindestens drei Messzeitpunkten Daten vorhanden.

4 In Bundesland Berlin ist es auch möglich nach der vierten Klasse auf eine weiterführende Schule zu wechseln. 
Tabelle 7. Teilnahme der Schülerinnen und Schüler an der Befragung.

\begin{tabular}{lcccccc}
\hline & Insgesamt & $\begin{array}{c}\text { eine } \\
\text { Welle }\end{array}$ & $\begin{array}{c}\text { Zwei } \\
\text { Wellen }\end{array}$ & $\begin{array}{c}\text { drei } \\
\text { Wellen }\end{array}$ & $\begin{array}{c}\text { vier } \\
\text { Wellen }\end{array}$ & $\begin{array}{c}\text { fünf } \\
\text { Wellen }\end{array}$ \\
\hline Baselinegruppe & 141 & 48 & 29 & 64 & - & - \\
& & $(34 \%)$ & $(21 \%)$ & $(45 \%)$ & 78 & 249 \\
Interventionsgruppe & 55 & 47 & 78 & 119 & 61 & $(45 \%)$ \\
Kontrollgruppe & \multirow{2}{*}{512} & 60 & 58 & 87 & 61 & 246 \\
& & $(11 \%)$ & $(11 \%)$ & $(17 \%)$ & $(11 \%)$ & $(48 \%)$ \\
\hline Insgesamt & \multirow{2}{*}{1.207} & 155 & 165 & 270 & 122 & 495 \\
& & $(13 \%)$ & $(14 \%)$ & $(22 \%)$ & $(10 \%)$ & $(41 \%)$ \\
\hline
\end{tabular}

Stichprobenmerkmale. Zum ersten Messzeitpunkt im November 2005 waren die Kinder in allen drei Untersuchungsgruppen im Mittel 8 Jahre und 11 Monate alt (siehe Tabelle 8). Im Mai 2009, am Ende der sechsten Klasse, war das mittlere Alter der Kinder 12 Jahre und 5 Monate. Unterschiede zwischen den Gruppen sind nicht festzustellen, weder im Quer- noch im Längsschnitt.

Insgesamt waren von den 1.207 Kindern 652 (52\%) männlichen Geschlechts, womit in der Gesamtstichprobe eine Geschlechtergleichverteilung annähernd erreicht werden konnte. In der Baselinegruppe (49\%) sind die Jungen jedoch im Vergleich zur Gesamtstichprobe leicht unter- sowie in der Kontrollgruppe (54\%) leicht überrepräsentiert.

Rund ein Viertel der Kinder (330; $27.5 \%$ ) kommt aus einem Elternhaus mit Migrationshintergrund, d. h. das Herkunftsland beider Elternteile ist nicht Deutschland, wobei in der Baselinegruppe mit 29.4 Prozent deutlich mehr Kinder einen Migrationshintergrund ${ }^{5}$ aufweisen. So sind es in dieser Gruppe auch 11.0 Prozent der Schülerinnen und Schüler, die angeben nicht in Deutschland geboren zu sein. In der Kontrollgruppe ist dies nur bei 6.1 Prozent und in der Interventionsgruppe bei 6.9 Prozent der Kinder der Fall.

5 Der Migrationsstatus wurde dabei wie folgt berechnet: Kommt ein Elternteil aus Deutschland, ist die Herkunft Deutsch. Kommen beide Eltern nicht aus Deutschland, ist die Herkunft Nicht-Deutsch. Gibt es einen Missing-Wert für eines der beiden Elternteile, wird die Herkunft des jeweils anderen verwendet. 
Tabelle 8. Stichprobenbeschreibung (Schülerinnen und Schüler nach Untersuchungsgruppe).

\begin{tabular}{|c|c|c|c|c|}
\hline & Gesamt & $\begin{array}{l}\text { Baseline- } \\
\text { gruppe }\end{array}$ & $\begin{array}{l}\text { Kontroll- } \\
\text { gruppe }\end{array}$ & $\begin{array}{l}\text { Interventions- } \\
\text { gruppe }\end{array}$ \\
\hline \multicolumn{5}{|l|}{$\overline{\text { Alter }}$} \\
\hline 3. Klasse (Halbjahr) & $8 ; 11$ & $8 ; 11$ & $8 ; 11$ & $8 ; 11$ \\
\hline 3. Klasse & $9 ; 6$ & - & $9 ; 6$ & $9 ; 6$ \\
\hline 4. Klasse & $10 ; 5$ & - & $10 ; 5$ & $10 ; 5$ \\
\hline 5. Klasse & $11 ; 5$ & $11 ; 5$ & $11 ; 6$ & $11 ; 5$ \\
\hline 6. Klasse & $12 ; 5$ & $12 ; 6$ & $12 ; 5$ & $12 ; 5$ \\
\hline Anteil männlich & $51.7 \%$ & $48.9 \%$ & $54.4 \%$ & $50.0 \%$ \\
\hline $\begin{array}{l}\text { Anteil Migrationshin- } \\
\text { tergrund }\end{array}$ & $27.5 \%$ & $29.6 \%$ & $27.4 \%$ & $27.2 \%$ \\
\hline $\begin{array}{l}\text { Geburtsland nicht } \\
\text { Deutschland }\end{array}$ & $6.6 \%$ & $11.0 \%$ & $6.1 \%$ & $6.9 \%$ \\
\hline \multicolumn{5}{|l|}{ Bildungsniveau $^{6}$} \\
\hline niedrig & $13.1 \%$ & $9.6 \%$ & $15.5 \%$ & $11.9 \%$ \\
\hline mittel & $33.1 \%$ & $30.7 \%$ & $35.7 \%$ & $31.2 \%$ \\
\hline hoch & $53.8 \%$ & $59.6 \%$ & $48.8 \%$ & $56.9 \%$ \\
\hline \multicolumn{5}{|l|}{ Wohlstandsindex ${ }^{7}$} \\
\hline Q 1 & $20.0 \%$ & $23.5 \%$ & $20.1 \%$ & $19.0 \%$ \\
\hline Q 2 & $20.0 \%$ & $16.9 \%$ & $20.9 \%$ & $19.9 \%$ \\
\hline Q 3 & $20.0 \%$ & $16.2 \%$ & $22.1 \%$ & $19.0 \%$ \\
\hline Q 4 & $19.8 \%$ & $15.4 \%$ & $19.3 \%$ & $22.2 \%$ \\
\hline Q 5 & $20.2 \%$ & $27.9 \%$ & $17.5 \%$ & $19.9 \%$ \\
\hline
\end{tabular}

Zur Schätzung des Bildungsniveaus im Elternhaus wurden die Angaben der Eltern zu ihrem schulischen Werdegang herangezogen: Das formale Bildungsniveau ist niedrig, wenn der höchste Bildungsabschluss beider Elternteile ein Hauptschulabschluss ist; Es ist mittel, wenn der höchste Bildungsabschluss mindestens eines Elternteils die Mittlere Reife ist; Es ist hoch, wenn der höchste Bildungsabschluss mindestens eines Elternteils das Abitur oder ein abgeschlossenes

6 Da die elterlichen Schulabschlüsse zu drei Messzeitpunkten (t 1, t 4, t 5 ) im Elternfragebogen erhoben wurden, wurde der höchste Schulabschluss der drei Messzeitpunkte zur Bestimmung des formalen familiären Bildungsniveaus herangezogen.

7 Da die Indikatoren der sozialen Lage zu drei Messzeitpunkten ( $1, \mathrm{t}$ 4, t 5) erhoben wurden, wurde zur Bestimmung des Wohlstandsindexes der Mittelwert (des Summenscores) aus allen drei Messzeitpunkten gebildet. Hier wurde bewusst zu Gunsten einer Fehlerkorrektur auf eine größere Differenzierung (nach „Auf“- und „Abstieg“) verzichtet. Für differenziertere Auswertungen ist es jedoch möglich die ermittelten Wohlstandsindizes der unterschiedlichen Messzeitpunkte einzubeziehen. 
Studium ist. Mehr als die Hälfte der Schülerinnen und Schüler der Berliner Stichprobe kommen aus einem Elternhaus mit hohem Bildungsniveau. Auf der anderen Seite leben 13 Prozent der Kinder in einem Elternhaus mit geringem Bildungsniveau (höchstens Hauptschulabschluss). Im Vergleich zur Gesamtstichprobe sind in der Baselinegruppe deutlich mehr Kinder aus einem Elternhaus mit hohem Bildungsniveau (59.6\%) vertreten. In der Kontrollgruppe sind diese jedoch im Vergleich zu den anderen beiden Untersuchungsgruppen mit 48.8 Prozent deutlich unterrepräsentiert. Somit ist beim Vergleich der Interventions- und Kontrollklassen ein Ungleichgewicht dahin gehend festzustellen, dass in Letzterer etwas mehr Kinder aus Familien mit niedrigem und mittlerem Bildungsniveau vertreten sind. Da das Bildungsniveau aber mithilfe der Daten aus dem Elternfragebogen geschätzt wurde, könnte dies möglicherweise auch mit dem Rücklauf der Elternfragebogen konfundiert sein, und zwar dann, wenn in den Kontrollklassen vor allem Eltern mit hohem Bildungsniveau keinen Elternfragebogen abgegeben haben. ${ }^{8}$

Zur Beschreibung des familiären Wohlstandes wurde ein Wohlstandsindex analog zum Jugendgesundheitssurvey (Hurrelmann, Klocke \& Ravens-Sieberer, 2003) berechnet, der sich aus den folgenden Items zusammensetzt: Wohnraumversorgung - „hast du zu Hause dein eigenes Zimmer?“ (ja, nein); Urlaubsreisen - „Wie häufig bist du in den letzten 12 Monaten mit deiner Familie in Urlaub gefahren? (überhaupt nicht, einmal, zweimal, mehr als zweimal); Automobilbesitz - „Besitzt deine Familie ein Auto?“ (nein, eins, zwei oder mehr); Computerbesitz - „Wie viele Computer besitzt deine Familie insgesamt zu Hause?“ (keinen, einen, zwei, mehr als zwei); Anzahl der Bücher im Haushalt - „Wie viele Bücher gibt es ungefähr bei dir zu Hause? Es zählen alle Bücher zu Hause (Zähle keine Zeitschriften, Zeitungen oder Schulbücher mit!).“ (keine/sehr wenige 0-10 Bücher, etwa ein Bücherbrett - 11-25 Bücher, etwa ein Regal - 26-100 Bücher, etwa zwei Regale - 101-200 Bücher, drei oder mehr Regale - mehr als 200 Bücher).

„Die Frage nach dem eigenen Zimmer [...] im Haushalt ist ein Indikator für die Wohnraumsituation. Hat [das Kind] kein eigenes Zimmer, so liegt eine Unterversorgung in diesem Bereich vor. [...] Die Anzahl der Urlaubsreisen im letzten Jahr misst ebenfalls die finanziellen Ressourcen des Haushalts, denn keine Urlaubsreise oder drei und mehr Urlaubsreisen spiegeln erhebliche Unterschiede in den Lebensbedingungen wie auch in der Lebensqualität der Kinder [...] wider. Die Anzahl der Automobile im Haushalt liefert einen stärker materiellen Hinweis auf die finanziellen Ressourcen des Haushalts. Zwei und mehr [PKW] pro Haushalt indizieren in den meisten Haushalten

8 Eine Erklärung für einen derartigen Effekt hätte der Autor jedoch nicht. 
ein gutes Haushaltseinkommen. Der Computerbesitz liefert sowohl einen Hinweis auf die Wohlstandsposition des Haushalts als auch einen Hinweis auf die Technikaufgeschlossenheit und Modernität. [...] Die Anzahl der Bücher wird als Indikator für das soziale Milieu gewertet, in dem die Kinder [...] aufwachsen.“ (Hurrelmann et al., 2003; S. 201f).

Die einzelnen Items wurden $\mathrm{z}$-standardisiert und zu einem additiven Index ${ }^{9} \mathrm{zu}-$ sammengefasst. Anhand der so berechneten Summenwerte wurde die Stichprobe in Quintile aufgeteilt. Die Verteilungseigenschaften der einzelnen Indikatoren der sozialen Lage mit einer positiven bzw. negativen Kumulierung an den beiden Polen des Wohlstandsindexes im Jugendgesundheitssurvey (vgl. Hurrelmann et al., 2003) konnte dabei repliziert werden (siehe Abbildung 3). Beim Vergleich der unterschiedlichen Untersuchungsgruppen fällt zunächst auf, dass in der Baselinegruppe die Quintile mit dem niedrigsten $(23.5 \%)$ und insbesondere dem höchsten (27.9\%) familiären Wohlstand zuungunsten der anderen Quintile leicht überrepräsentiert sind. Letzteres ist in der Kontrollgruppe (17.5\%) leicht unterrepräsentiert. Trotz dieser geringen Unterschiede sind die Quintile in den verschiedenen Untersuchungsgruppen annähernd gleich verteilt (die größten Unterschiede sind dabei in der Baselinegruppe zu beobachten, was hier vor allem der kleinen Stichprobengröße dieser Teilstichprobe geschuldet ist). Zusammengefasst sind insbesondere Interventions- und Kontrollklassen hinsichtlich ihrer Schülerzusammensetzung vergleichbar, eine Tatsache, die vor allem durch die randomisierte Gruppenzuweisung erreicht werden konnte.

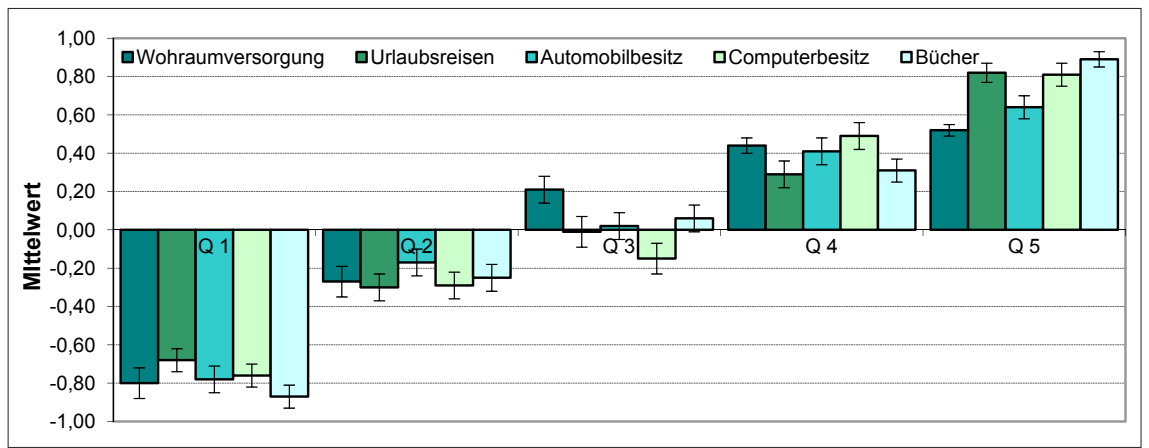

Abbildung 3. Ausprägung der Indikatoren der sozialen Lage (t 5) nach Wohlstandsquintilen.

9 Maximal zwei der fünf Items durften dabei für eine Person einen Missing-Wert aufweisen. $\mathrm{Zu}$ den Vor- und Nachteilen dieses Indexes siehe Jugendgesundheitssurvey (Hurrelmann et al., 2003). 
Eingehendere Betrachtung der Stichprobenmobilität. Von besonderem Interesse ist die Frage, ob diejenigen Schülerinnen und Schüler, die aus einem der obigen Gründe (Umzug, Klassenwechsel, etc.) nicht mehr in der Untersuchungsstichprobe sind (insbesondere diejenigen, die nach der vierten oder fünften Klasse auf eine weiterführende Schule gewechselt haben), sich hinsichtlich bestimmter sozial-struktureller Merkmale systematisch von denjenigen unterscheiden, die in der Stichprobe verblieben sind. Gibt es demnach einen selektiven Stichprobenausfall? Gleichzeitig ist auf der anderen Seite von Interesse, ob diejenigen Schülerinnen und Schüler die neu zur Untersuchungsstichprobe hinzugekommen sind (insbesondere nach der vierten und fünften Klasse), sich ebenfalls hinsichtlich bestimmter sozial-struktureller Merkmale systematisch unterscheiden. Gibt es demnach auch einen selektiven Stichprobenzuwachs?

Betrachtet man die Stichprobe getrennt nach den Kategorien besucht die Klasse noch, umgezogen (nach 3., 4. oder 5. Klasse), Wechsel auf weiterführende Schule (nach 4. oder 5. Klasse) sowie zur Stichprobe nach 4. oder 5. Klasse hinzugekommen, müssen beide Fragen eindeutig bejaht werden (siehe Tabelle 9).

So sind diejenigen Schülerinnen und Schüler, die nicht mehr an der Befragung teilgenommen haben, im Vergleich zu den in der Stichprobe verbliebenen Schülerinnen und Schülern zu einem größeren Teil Kinder aus Familien mit einem niedrigen oder hohem familiären Bildungsniveau sowie höherem familiären Wohlstand. Differenziert man die aus der Stichprobe ausgeschiedenen Schülerinnen und Schüler nach dem Grund (Umzug, Wechsel auf weiterführende Schule), so sind diejenigen Schülerinnen und Schüler, die aufgrund eines Umzuges nicht mehr an der Befragung teilgenommen haben, im Vergleich zu den in der Stichprobe verbliebenen Schülerinnen und Schülern zu einem größeren Teil Kinder aus Familien mit einem niedrigen oder mittleren familiären Bildungsniveau sowie geringerem familiären Wohlstand. Die Schülerinnen und Schüler, die nach der vierten Klasse auf eine weiterführende Schule gewechselt sind, kommen dagegen im Vergleich zu den beiden anderen Gruppen deutlich häufiger aus Familien mit einem hohen Bildungsniveau und hohem familiären Wohlstand. In beiden Gruppen ist zusätzlich der Anteil der Jungen im Vergleich zu den in der Klasse verbliebenen Schülern leicht erhöht sowie der Anteil an Kindern aus Familien mit Migrationshintergrund verringert.

Auf der anderen Seite kommen diejenigen Schülerinnen und Schüler, die neu in die Stichprobe hinzugekommen sind, zu einem größeren Anteil aus Familien mit einem niedrigen familiären Bildungsniveau und geringem familiären Wohlstand. Darüber hinaus ist der Anteil der Jungen sowie an Kindern aus Familien mit Migrationshintergrund erhöht.

Zusammengefasst kann somit sowohl am unteren (Umzug und dies vor allem in der Kontrollgruppe) als auch am oberen Ende (Schulwechsel) der Verteilung 
ein Stichprobenausfall festgestellt werden, der sich durch den beidseitigen Wegfall zwar weniger auf den Mittelwert auswirkt, aber für eine insgesamt geringere Varianz in den Daten sorgt. Der Wegfall am unteren Ende der Verteilung wird durch einen selektiven Stichprobenzuwachs zum Teil ausgeglichen.

Tabelle 9. Stichprobenbeschreibung nach Teilnahmestatus.

\begin{tabular}{|c|c|c|c|c|c|}
\hline & \multirow{2}{*}{$\begin{array}{l}\text { besucht die } \\
\text { Klasse } \\
\text { noch }\end{array}$} & \multicolumn{3}{|c|}{ Aus der Stichprobe ausgeschieden } & \multirow{2}{*}{$\begin{array}{l}\text { Neu in der } \\
\text { Stichprobe }\end{array}$} \\
\hline & & Gesamt & Umgezogen & Wechsel & \\
\hline $\mathbf{N}$ & $832 *$ & $242 *$ & $127 *$ & $100 *$ & 264 \\
\hline Anteil männlich & $50.4 \%$ & $57.0 \%$ & $55.1 \%$ & $57.0 \%$ & $55.3 \%$ \\
\hline $\begin{array}{l}\text { Anteil Migrati- } \\
\text { onshintergrund }\end{array}$ & $28.3 \%$ & $23.4 \%$ & $22.6 \%$ & $24.0 \%$ & $33.1 \%$ \\
\hline \multicolumn{6}{|l|}{ Bildungsniveau } \\
\hline niedrig & $12.6 \%$ & $15.6 \%$ & $19.5 \%$ & $3.7 \%$ & $15.4 \%$ \\
\hline mittel & $35.6 \%$ & $26.7 \%$ & $37.9 \%$ & $17.3 \%$ & $32.1 \%$ \\
\hline hoch & $51.8 \%$ & $57.8 \%$ & $42.5 \%$ & $79.0 \%$ & $52.5 \%$ \\
\hline \multicolumn{6}{|l|}{ Wohlstandsindex } \\
\hline Q 1 & $21.0 \%$ & $18.3 \%$ & $24.3 \%$ & $10.8 \%$ & $25.9 \%$ \\
\hline Q 2 & $21.0 \%$ & $17.4 \%$ & $22.4 \%$ & $9.7 \%$ & $16.8 \%$ \\
\hline Q 3 & $19.8 \%$ & $17.4 \%$ & $19.6 \%$ & $12.9 \%$ & $25.0 \%$ \\
\hline Q 4 & $20.0 \%$ & $22.1 \%$ & $16.8 \%$ & $30.1 \%$ & $17.7 \%$ \\
\hline Q 5 & $18.3 \%$ & $24.9 \%$ & $16.8 \%$ & $36.6 \%$ & $14.7 \%$ \\
\hline
\end{tabular}

Anmerkung. Umgezogen $=$ Umgezogen nach der 3., 4. oder 5. Klasse. Wechsel $=$ Wechsel auf eine weiterführende Schule nach der 4. oder 5. Klasse. Neu in der Stichprobe = Zur Stichprobe hinzugekommen nach der 4. oder 5. Klasse. *Kinder zu denen Angaben aus dem Lehrerfragebogen vorliegen.

Angesichts der berichteten Stichprobenmerkmale (Wohlstands- und Bildungsniveau im Elternhaus, Rücklauf- und Teilnahmequoten, Stichprobenmortalität, Geschlechterverteilung, Anteil an Kindern mit Migrationshintergrund, Vergleichbarkeit der Untersuchungsgruppen) konnte insgesamt für den Berliner Längsschnitt Medien eine sehr gute Stichprobenqualität erreicht werden.

\subsubsection{Eltern}

Untersuchungsteilnahme. Zu Beginn der dritten Klasse (t 1) sowie jeweils am Ende der vierten ( $\mathrm{t}$ 3), fünften ( $\mathrm{t} 4$ ) und sechsten (t 5) Klasse kam neben dem Schüler- und Lehrerfragebogen auch ein Elternfragebogen zum Einsatz, der über 
die Kinder an die Eltern verteilt wurde. Somit ist die Teilnahme der Eltern immer an die Teilnahme des eigenen Kindes an der Befragung gekoppelt. Die 1.085 Eltern, die sich insgesamt an der Befragung beteiligten, verteilen sich dabei wie folgt auf die unterschiedlichen Messzeitpunkte (siehe Tabelle 10). 270 $(22 \%)$ der Eltern beteiligten sich an allen vier Befragungswellen, an denen ein Elternfragebogen ausgegeben wurde. 237 Eltern (20\%) beteiligten sich an drei Befragungswellen, 264 (22\%) an zwei Befragungswellen sowie $264(22 \%)$ an nur einer Befragungswelle. 122 Eltern (10\%) haben zu keinem der drei Messzeitpunkte einen Fragebogen ausgefüllt. 9 dieser Eltern hatten aber gar keine Möglichkeit zum Ausfüllen, da ihre Kinder sich nur an der zweiten Befragungswelle beteiligten, an der keine Elternbefragung durchgeführt wurde. Die Unterschiede in den Teilnahmequoten sind dabei zwischen den Gruppen, wie auch bei den Kindern, mit Ausnahme einer höheren nicht-Teilnahme in der Baselinegruppe, nur geringfügig. So haben in allen Gruppen etwa ein Viertel (zwischen $24 \%$ und $27 \%$ ) der Eltern an allen ihnen möglichen Messzeitpunkten teilgenommen. Insgesamt liegen somit zu 305 Eltern (25\%) hinsichtlich der Messzeitpunkte komplette Datensätze vor, was bedeutet, dass für ein Viertel der Kinder auch die kompletten Informationen aus den Elternfragebögen zur Verfügung stehen. Setzt man dies zu denjenigen Kindern in Beziehung, die sich an allen Messzeitpunkten an der Befragung beteiligt haben, so sind bei 54 Prozent der Kinder der Stichprobe komplette Datensätze inklusive Elternfragebögen vorhanden. Für insgesamt 504 der Kinder (42\%) liegen hinsichtlich der Elternfragebögen entweder komplette Datensätze vor oder es sind in der Interventions- und Kontrollgruppe zu mindestens drei Messzeitpunkten Daten erhoben worden. Für gut zwei Drittel (68\%) der Kinder liegen zu mindestens zwei Messzeitpunkten komplette Datensätze inklusive Elternfragebögen vor.

Tabelle 10. Teilnahme der Eltern an der Befragung.

\begin{tabular}{lcccccc}
\hline & $\begin{array}{c}\text { Insge- } \\
\text { samt }\end{array}$ & 0 Wellen & $\begin{array}{c}\text { eine } \\
\text { Welle }\end{array}$ & $\begin{array}{c}\text { Zwei } \\
\text { Wellen }\end{array}$ & $\begin{array}{c}\text { drei } \\
\text { Wellen }\end{array}$ & $\begin{array}{c}\text { vier } \\
\text { Wellen }\end{array}$ \\
\hline Baselinegruppe & 141 & 26 & 50 & 30 & 35 & - \\
Interventions- & 554 & 44 & 98 & 163 & 101 & 148 \\
gruppe & & $(8 \%)$ & $(18 \%)$ & $(29 \%)$ & $(18 \%)$ & $(27 \%)$ \\
Kontrollgruppe & 512 & 52 & 116 & 121 & 101 & 122 \\
& & $(10 \%)$ & $(23 \%)$ & $(24 \%)$ & $(20 \%)$ & $(24 \%)$ \\
\hline Insgesamt & 1.207 & 122 & 264 & 314 & 237 & 270 \\
& & $(10 \%)$ & $(22 \%)$ & $(26 \%)$ & $(20 \%)$ & $(22 \%)$ \\
\hline
\end{tabular}

Stichprobenmerkmale. Zum ersten Messzeitpunkt im November 2005 waren die Elternteile, die sich an der Befragung beteiligt haben, in der Interventions- 
gruppe im Durchschnitt 37 Jahre sowie in der Baseline- und Kontrollgruppe 38 Jahre alt (siehe Tabelle 11). Über die Messzeitpunkte ändert sich bei gleichbleibender Standardabweichung (6 Jahre) die Altersstruktur der Eltern trotz oben berichteter Stichprobenfluktuation insgesamt und im Vergleich zwischen den Gruppen nicht. So waren zum letzten Messzeitpunkt im Mai 2009 die Elternteile, die sich an der Befragung beteiligt haben, in der Interventionsgruppe im Durchschnitt 41 Jahre sowie in der Baseline- und Kontrollgruppe 42 Jahre alt.

Der Anteil der Väter, die sich an der Befragung beteiligt haben, ist relativ gering und schwankt je nach Untersuchungsgruppe und Messzeitpunkt zwischen 14 und 23 Prozent, wobei diese beiden Pole durch eine deutliche Abnahme des Väteranteils in der Baselinegruppe von t 1 zu t 5 markiert werden. Kontroll- und Interventionsgruppe unterscheiden sich insgesamt nur marginal voneinander. In der Kontrollgruppe ist jedoch wie in der Baselinegruppe ein leichter Abfall, in der Interventionsgruppe ein leichter Anstieg der sich beteiligenden Väter zu beobachten.

Rund drei Viertel der Eltern (zwischen $70 \%$ und $80 \%$, im Mittel 74\%) gab im Fragebogen an, in Deutschland geboren zu sein. Dieser Anteil bleibt, abgesehen von einer Abnahme in der Baselinegruppe über die Messzeitpunkte relativ stabil, was zumindest in den Kontroll- und Interventionsklassen nicht auf eine selektive Nichtteilnahme von Eltern mit oder ohne Migrationshintergrund zu einem der Messzeitpunkte schließen lässt. Des Weiteren passen diese Angaben sehr gut zu dem aus den Kinderangaben gefolgerten Migrationsstatus der Familie. Es wurde aufgrund der Kinderangaben rund ein Viertel (27.5\%) der Kinder identifiziert, von welchen das Herkunftsland beider Elternteile nicht Deutschland war.

Betrachtet man die Formen familiären Zusammenlebens, so zeigt sich, dass über alle Gruppen zwischen 56 und 58.5 Prozent der ausfüllenden Eltern zum Zeitpunkt der Befragung verheiratet waren und mit ihrem Partner zusammenlebten. Dies bleibt über den gesamten Befragungszeitraum insgesamt und innerhalb der Untersuchungsgruppen relativ stabil. Lediglich der Anteil der Geschiedenen nahm von 12.5 Prozent zu Beginn der dritten Klasse auf 14.6 Prozent am Ende der fünften Klasse zu. Im Vergleich der Untersuchungsgruppen ist festzustellen, dass sowohl in der Baseline- als auch in der Kontrollgruppe die Eltern, die verheiratet waren und zusammenlebten, von Beginn an zuungunsten der Geschiedenen leicht überrepräsentiert waren. Wie für die Kinder so gilt jedoch auch für die befragten Eltern, dass insbesondere Interventions- und Kontrollklassen hinsichtlich ihrer Elternzusammensetzung vergleichbar sind. 
Tabelle 11. Stichprobenbeschreibung (Eltern nach Untersuchungsgruppe).

\begin{tabular}{lccc} 
Gesamt & $\begin{array}{c}\text { Baseline- } \\
\text { gruppe }\end{array}$ & $\begin{array}{c}\text { Kontroll- } \\
\text { gruppe }\end{array}$ & $\begin{array}{c}\text { Interventions- } \\
\text { gruppe }\end{array}$ \\
\hline 3. Klasse Halbjahr
\end{tabular}

\section{Klasse Halbjahr}

\section{Alter (SD)}

\section{Anteil männlich}

Geburtsland D

\section{Familienstand}

verheiratet zusammenle-
bend
verheiratet, getrennt lebend
verwitwet
geschieden
ledig
Lebensgemeinschaft

\section{Klasse}

\section{Alter (SD)}

\section{Anteil männlich}

Geburtsland D

$58.5 \%$

$6.2 \%$

$0.7 \%$

$12.5 \%$

$19.2 \%$

$2.9 \%$

$39(6)$

$17 \%$

$73 \%$

\section{Familienstand}

verheiratet zusammenlebend

$56.1 \%$

$38(6)$

$38(6)$

$37(6)$

$17 \% \quad 23 \%$

$80 \%$

$18 \%$

$74 \%$

$16 \%$

$74 \%$

verheiratet, getrennt lebend

$7.1 \%$

verwitwet

$1.0 \%$

geschieden

$14.5 \%$

ledig

$18.9 \%$

Lebensgemeinschaft

$2.4 \%$

\section{Klasse}

Alter (SD)

Anteil männlich

Geburtsland D

Familienstand

verheiratet zusammenlebend

verheiratet, getrennt lebend

$58.0 \%$

$40(6)$

$42(5)$

$40(6)$

$40(6)$

$19 \%$

$20 \%$

$73 \%$

$74 \%$

$63.2 \%$

$60.2 \%$

$54.4 \%$

verwitwet

$6.2 \%$

$7.4 \%$

$6.7 \%$

$5.4 \%$

$1.4 \%$

geschieden

$14.9 \%$

ledig

$17.7 \%$

Lebensgemeinschaft

$2.9 \%$

$0.8 \%$

$1.5 \%$

$10.3 \%$

$13.4 \%$

$17.6 \%$

$16.2 \%$

$16.9 \%$

$18.8 \%$

$2.0 \%$

$2.3 \%$ 


\begin{tabular}{lcccc}
\hline & Gesamt & $\begin{array}{c}\text { Baseline- } \\
\text { gruppe }\end{array}$ & $\begin{array}{c}\text { Kontroll- } \\
\text { gruppe }\end{array}$ & $\begin{array}{c}\text { Interventions- } \\
\text { gruppe }\end{array}$ \\
\hline $\begin{array}{lccc}\text { 6. Klasse } \\
\text { Alter (SD) }\end{array}$ & $42(6)$ & $42(5)$ & $42(7)$ & $41(5)$ \\
$\begin{array}{l}\text { Anteil männlich } \\
\text { Geburtsland D }\end{array}$ & $18 \%$ & $14 \%$ & $16 \%$ & $20 \%$ \\
Familienstand & $74 \%$ & $72 \%$ & $75 \%$ & $74 \%$ \\
$\quad \begin{array}{l}\text { verheiratet zusammenle- } \\
\text { bend }\end{array}$ & $58.4 \%$ & $59.3 \%$ & $58.6 \%$ & $58.0 \%$ \\
$\quad \begin{array}{l}\text { verheiratet, getrennt lebend } \\
\quad \text { verwitwet }\end{array}$ & $6.2 \%$ & $6.8 \%$ & $7.3 \%$ & $5.3 \%$ \\
$\quad \begin{array}{l}\text { geschieden } \\
\text { ledig }\end{array}$ & $1.2 \%$ & $5.1 \%$ & $0.5 \%$ & $1.1 \%$ \\
$\quad$ Lebensgemeinschaft & $14.6 \%$ & $11.9 \%$ & $14.1 \%$ & $15.5 \%$ \\
\hline
\end{tabular}

\subsubsection{Lehrkräfte}

Zusätzlich zu den Angaben der Kinder und Eltern füllte jede Lehrkraft während des Klasseninterviews einen Fragebogen aus, in dem auch Angaben zur eigenen Person (Geschlecht, Alter, Funktion in der Klasse, unterrichtete Fächer) gemacht werden sollten (siehe Tabelle 12). Der Kontakt zu den befragten Klassen wurde als Teil des organisatorischen Ablaufs zu jedem Messzeitpunkt über den Klassenlehrer oder die Klassenlehrerin hergestellt. So ist es auch nicht verwunderlich, dass fast ausschließlich Klassenlehrkräfte den Lehrerfragebogen ausfüllten, da diese die Befragung in ihren eigenen Stunden in der Klasse organisierten. Ein Teil der Lehrkräfte war dabei als Fachlehrer oder Fachlehrerin in der Klasse, nur einzelne als Beratungslehrer oder Beratungslehrerin tätig.

Unter den 89 Lehrkräften, die sich an mindestens einer der Befragungswellen beteiligt haben, ist ein deutlicher Überhang weiblicher Lehrkräfte zu beobachten. So waren zum ersten Messzeitpunkt im November 2005 nur drei Lehrer (6\%) an der Befragung beteiligt und füllten einen Fragebogen aus. Bis zur letzten Befragung hat sich das Geschlechterverhältnis jedoch zugunsten der Lehrer verbessert. So waren am Ende der sechsten Klasse 12 Prozent der ausfüllenden Lehrkräfte männlichen Geschlechts.

Die Altersstruktur der Lehrkräfte ändert sich trotz einiger Lehrkraftwechsel mit einem Mittelwert von 48 Jahren in der dritten und 51 Jahren in der sechsten Klasse sowie einer Standardabweichung von 7/8 Jahren entsprechend der durch den Längsschnitt vergangenen Zeit. 
Tabelle 12. Stichprobenbeschreibung Lehrkräfte.

\begin{tabular}{|c|c|c|c|c|c|}
\hline & t 1 & $t^{2}$ & t 3 & $\mathrm{t} 4$ & t 5 \\
\hline Klassenlehrer/in & $97.8 \%$ & $100 \%$ & $97.4 \%$ & $97.5 \%$ & $97.5 \%$ \\
\hline Fachlehrer/in & $32.6 \%$ & $46.2 \%$ & $38.5 \%$ & $57.5 \%$ & $63.4 \%$ \\
\hline Vertretungslehrer/in & $2.2 \%$ & $2.6 \%$ & $2.6 \%$ & - & $4.9 \%$ \\
\hline Beratungslehrer/in & - & $2.6 \%$ & - & $2.5 \%$ & - \\
\hline Geschlecht weiblich & $94 \%$ & $92 \%$ & $80 \%$ & $75 \%$ & $78 \%$ \\
\hline Alter & $48(8)$ & $49(8)$ & $48(8)$ & $49(7)$ & $51(7)$ \\
\hline $\begin{array}{l}\text { Kennt Klasse seit __ Mona- } \\
\text { ten }\end{array}$ & $20(12)$ & $26(11)$ & $22(15)$ & $28(18)$ & $38(18)$ \\
\hline Kennt Schüler nicht gut & - & - & - & $4 \%$ & $5.1 \%$ \\
\hline Kennt Schüler ziemlich gut & $63.6 \%$ & $48.7 \%$ & $44.7 \%$ & $42.5 \%$ & $30.8 \%$ \\
\hline Kennt Schüler sehr gut & $36.4 \%$ & $51.3 \%$ & $55.3 \%$ & $52.5 \%$ & $64.1 \%$ \\
\hline $\begin{array}{l}\text { Anzahl Unterrichtsstunden } \\
\text { in der Woche }\end{array}$ & $17(5)$ & $17(5)$ & $16(4)$ & $14(5)$ & $14(4)$ \\
\hline
\end{tabular}

Anmerkung. Alter in Jahren (Standardabweichung).

Im Mittel gaben die Lehrkräfte zu Beginn der dritten Klasse an, ihre Klasse seit ca. 20 Monaten zu kennen. Eine gewisse Kenntnis der Kinder ist unter anderem deshalb von Bedeutung, da wir von den Lehrkräften insbesondere bei der ersten Befragung neben Noten auch Einschätzungen zum Lern- und Arbeitsverhalten der Kinder erfragten. Bei der Befragung in der vierten Klasse ist ein kleiner Einbruch in der mittleren Kenntnis zu beobachten, verursacht durch den häufigen Wechsel des Klassenlehrers oder der Klassenlehrerin nach der dritten Klasse. Bis zum Ende der sechsten Klasse nimmt diese Kenntnis aber wieder deutlich $\mathrm{zu}$, was wiederum auf eine gewisse Konstanz der Lehrkräfte hinweist. Gefragt, wie gut sie die Klasse kennen, gaben fast alle Lehrkräfte an die Klasse ziemlich oder sehr gut zu kennen. Die Zahl der Unterrichtsstunden, die pro Woche in der Klasse verbracht werden, nimmt dabei über die Jahre von 17 auf 14 Stunden bei gleichbleibender Standardabweichung von 4 bis 5 Stunden ab.

\subsection{Beschreibung der Skalen- und Testinstrumente}

Im Folgenden sollen die eingesetzten Testinstrumente und Skalen, welche die Grundlage der Ergebnisdarstellungen des Berliner Längsschnitt Medien bilden, getrennt nach Instrumenten und Skalen des KFN-Schülerfragebogens (1.5.1), standardisierten Testverfahren (1.5.2) sowie Instrumenten und Skalen des KFNElternfragebogens (1.5.3) dargestellt werden. Auf eine gesonderte Darstellung 
der einfachen Erfassung der Noten bzw. Schullaufbahnempfehlungen im Lehrerfragebogen wird verzichtet. Die Erfassung der Rückmeldung zum Medienunterricht ist in Kapitel 7 (Medienprävention und-intervention) beschrieben. Die Beschreibungen der Indizes Bildungshintergrund und Wohlstand im Elternhaus finden sich unter der Stichprobenbeschreibung der Schülerinnen und Schüler in Kapitel 1.4.1. Zur schnellen Übersicht ist durch die Symbole (10345 jeweils gekennzeichnet, zu welchen Messzeitpunkten die jeweiligen Instrumente, Skalen und Tests zum Einsatz kamen.

\subsubsection{Instrumente und Skalen des KFN-Schülerfragebogens}

\section{Freizeitverhalten}

12345

Im Fragebogen zum Halbjahr der dritten Klasse wurde das Freizeitverhalten der Schülerinnen und Schüler in einem offenen Antwortformat mit der sog. GesternFrage erhoben. Aufgrund der Überlegung, dass eine reine Erfassung täglicher oder wöchentlicher Durchschnittszeiten mittels eines Fragebogens nur zu groben Schätzungen mit einer großen Fehleranfälligkeit führen würde, wurde den Grundschülerinnen und Grundschülern ein Plan mit dem Anker des gestrigen Tages vorgelegt. Folgende Tätigkeiten wurden dabei abgefragt: „In der Schule gewesen“, „Im Hort oder bei der Tagesmutter gewesen“, „Computer- oder Videospiele gespielt", „,gelesen“, „Fernsehen geschaut“, „Video oder DVD geschaut“, „Mit anderen Kindern draußen gespielt“, „Mit anderen Kindern drinnen gespielt", „Alleine drinnen gespielt", ,Mit Eltern oder anderen Erwachsenen etwas gemacht" (siehe Abbildung 4). Um eine möglichst genaue Schätzung des Wochendurchschnitts zu erhalten, wurde bei der Planung der Befragungstermine darauf geachtet, die Klasseninterviews über die ganze Woche zu verteilen. Die einzigen Tage, die auf diese Weise nicht erfasst werden konnten, waren der Freitag sowie der Samstag, da an den darauf folgenden Tagen in der Schule nicht befragt werden konnte. 


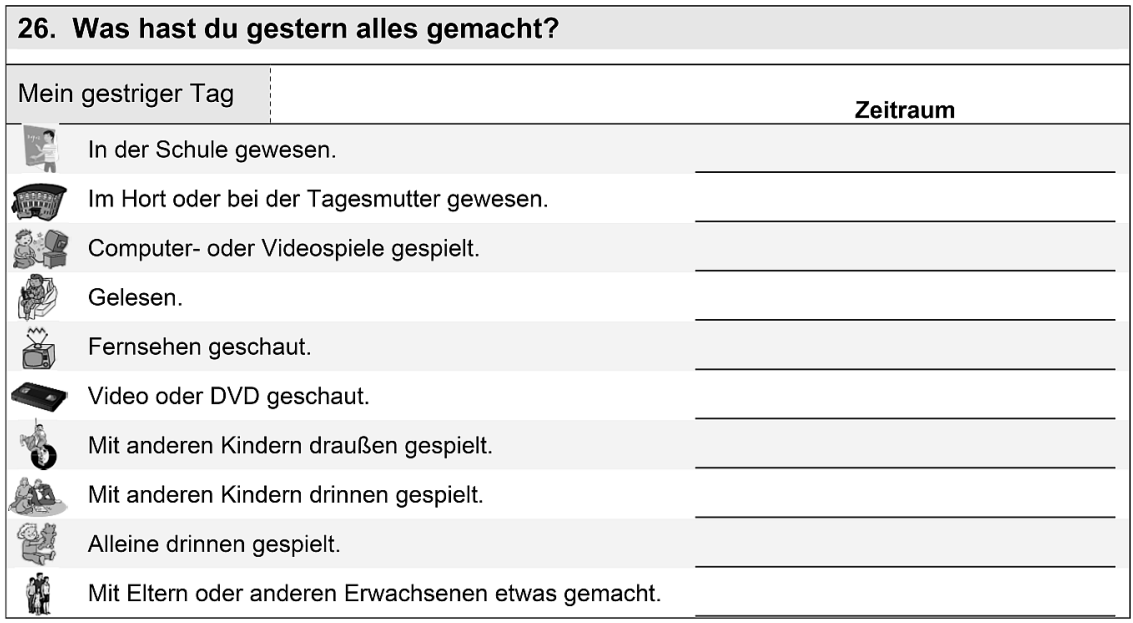

Abbildung 4. „Gestern-Frage“ zur Erhebung des Freizeitverhaltens in der dritten Klasse (Halbjahr).

Ab dem zweiten Messzeitpunkt wurde die Gestern-Frage zur Erhebung der Mediennutzungszeiten der Kinder überarbeitet und von einem offenen Antwortformat in die geschlossene Form eines Stundenplanes übertragen, sodass sie eher mit einem thematisch gegliederten Tagebuch des gestrigen Tages zu vergleichen ist (siehe Abbildung 5). Ausschlaggebend hierfür war die schlechte Datenqualität des offenen Antwortformats. So wurde z. B. selten eine Tätigkeit mehrmals angegeben, obwohl dies explizit in der Instruktion ermöglicht wurde. In dem thematisch gegliederten Tagebuch konnten die Kinder für die Tätigkeiten „In der Schule gewesen“, „Im Hort oder bei der Tagesmutter gewesen“, „Computeroder Videospiele gespielt“, „gelesen“, „Fernsehen geschaut“, „Video oder DVD geschaut", „Mit anderen Kindern draußen gespielt"“, „Mit anderen Kindern drinnen gespielt", „Alleine drinnen gespielt" ${ }^{10}$ „Mit Eltern oder anderen Erwachsenen etwas gemacht“, „das Internet genutzt (nur 5. und 6. Klasse)“ Halbstunden genau markieren, inwiefern sie diese am Tag vor der Befragung ausgeübt hatten. Dabei konnten sich zwei Tätigkeiten auch überlappen (z. B. Fernsehen und mit anderen drinnen spielen) und einer Tätigkeit mehrmals am Tag nachgegangen werden.

10 „Mit anderen Kindern draußen gespielt“, „Mit anderen Kindern drinnen gespielt“ und „Alleine drinnen gespielt" wurde ab der 4 . Klasse zu ,,mit Kindern drinnen oder draußen gespielt" zusammengefasst. 


\begin{tabular}{|c|c|c|c|c|c|c|c|c|c|c|}
\hline \multicolumn{2}{|c|}{$\begin{array}{l}\text { Mein } \\
\text { gestriger } \\
\text { Tag }\end{array}$} & $\begin{array}{c}\text { In der } \\
\text { Schule } \\
\text { gewesen }\end{array}$ & $\begin{array}{c}\text { Im Hort } \\
\text { oder bei } \\
\text { der } \\
\text { Tagesmut- } \\
\text { ter } \\
\text { gewesen }\end{array}$ & $\begin{array}{l}\text { Computer- } \\
\text { oder Video- } \\
\text { spiele } \\
\text { gespielt }\end{array}$ & $\begin{array}{l}\text { Bücher } \\
\text { gelesen }\end{array}$ & $\begin{array}{c}\text { Fernsehen } \\
\text { geschaut }\end{array}$ & $\begin{array}{l}\text { Video oder } \\
\text { DVD } \\
\text { geschaut }\end{array}$ & $\begin{array}{l}\text { Das } \\
\text { Internet } \\
\text { genutzt }\end{array}$ & $\begin{array}{l}\text { Mit Kindern } \\
\text { drinnen } \\
\text { oder } \\
\text { draußsen } \\
\text { gespielt }\end{array}$ & $\begin{array}{l}\text { Mit Eltern } \\
\text { odder } \\
\text { anderen } \\
\text { Erwachse- } \\
\text { nen etwas } \\
\text { gemacht }\end{array}$ \\
\hline \multirow{12}{*}{ 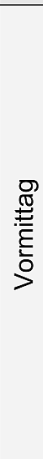 } & $7^{00}-7^{30}$ & $\square$ & $\square$ & $\square$ & $\square$ & $\square$ & $\square$ & $\square$ & $\square$ & $\square$ \\
\hline & $7^{30}-8^{00}$ & $\square$ & $\square$ & $\square$ & $\square$ & $\square$ & $\square$ & $\square$ & $\square$ & $\square$ \\
\hline & $8^{00}-8^{30}$ & $\square$ & $\square$ & $\square$ & $\square$ & $\square$ & $\square$ & $\square$ & $\square$ & $\square$ \\
\hline & $8^{30}-9^{00}$ & $\square$ & $\square$ & $\square$ & $\square$ & $\square$ & $\square$ & $\square$ & $\square$ & $\square$ \\
\hline & $9^{00}-9^{30}$ & $\square$ & $\square$ & $\square$ & $\square$ & $\square$ & $\square$ & $\square$ & $\square$ & $\square$ \\
\hline & $9^{30}-10^{00}$ & $\square$ & $\square$ & $\square$ & $\square$ & $\square$ & $\square$ & $\square$ & $\square$ & $\square$ \\
\hline & $10^{00}-10^{30}$ & $\square$ & $\square$ & $\square$ & $\square$ & $\square$ & $\square$ & $\square$ & $\square$ & $\square$ \\
\hline & $10^{30}-11^{00}$ & $\square$ & $\square$ & $\square$ & $\square$ & $\square$ & $\bar{\square}$ & $\square$ & $\bar{\square}$ & $\square$ \\
\hline & $11^{00}-11^{30}$ & $\square$ & $\square$ & $\square$ & $\square$ & $\square$ & $\square$ & $\square$ & $\square$ & $\square$ \\
\hline & $11^{30}-12^{00}$ & $\square$ & $\square$ & $\square$ & $\square$ & $\square$ & $\square$ & $\square$ & $\square$ & $\square$ \\
\hline & $12^{00}-12^{30}$ & $\square$ & $\square$ & $\square$ & $\square$ & $\square$ & $\square$ & $\square$ & $\square$ & $\square$ \\
\hline & $12^{30}-13^{00}$ & $\square$ & $\square$ & $\square$ & $\square$ & $\square$ & $\square$ & $\square$ & $\square$ & $\square$ \\
\hline
\end{tabular}

Abbildung 5. „Gestern-Frage“ (nur Vormittag; Erfassung bis 23.00) zur Erhebung des Freizeitverhaltens (3. bis 6. Klasse).

\section{Medienausstattung}

10845

In allen fünf Befragungswellen wurde die Geräteausstattung im Kinderzimmer (siehe Abbildung 6) sowie des Elternhauses (,woanders bei euch zu Hause“) in zwei getrennten Items erhoben.

12. Gib bitte an, ob du folgende Geräte bei dir im Zimmer hast.

$\begin{array}{lll} & \text { Ja } & \text { Nein } \\ \text { Fernseher } & \square & \square \\ \text { Spielkonsole } & \square & \square \\ \text { DVD-Player/Videorekorder } & \square & \square \\ \text { Computer } & \square & \square \\ \text { Internetanschluss } & \square & \square\end{array}$

Abbildung 6. Erhebung der Medienausstattung.

\section{Mediennutzungszeiten}

12845

Neben der Erhebung der Mediennutzungszeiten in der Gestern-Frage, wurden diese zusätzlich mit der sog. Schätzfrage (siehe Abbildung 7) getrennt für das 
Fernsehen, das Computerspielen und die Internetnutzung (nur 5. und 6. Klasse) erfasst. In der Schätzfrage werden die Kinder darum gebeten, ihre durchschnittliche Fernseh-, Computerspiel-, oder Internetnutzungszeit für einen Schultag und einen Samstag anzugeben. ${ }^{11}$ Da diese Einschätzung ein gewisses Abstraktionsvermögen vom Beantwortenden verlangt, wurden den Kindern zur Erleichterung sieben Antwortkategorien mit einer halbstündigen Differenzierung im unteren Nutzungsbereich vorgegeben. Mit der Kategorie ,ich schaue nicht fern“, „ich spiele nicht" oder ,ich nutze das Internet nicht" hatten die Kinder auch die Möglichkeit zu kennzeichnen, ob sie an einem Schultag oder an einem Samstag typischerweise gar nicht Fernsehen, gar keine Computerspiele spielen oder gar nicht das Internet nutzen. Die Schätzfrage hat zunächst den Nachteil, dass es zum einen die Nutzungszeiten im Gruppenmittel leicht überschätzt und daher gegenüber der Frage, wie viel am vorangegangen Tag gespielt wurde (vgl. GesternFrage), zu leicht erhöhten Gruppenmittelwerten führt. Zum anderen wird im oberen Nutzungsspektrum nicht mehr differenziert, wobei diese Differenzierung angesichts der aus Gründen der Vergleichbarkeit zu Beginn der dritten Klasse sehr hoch angesetzten Zeiten verzichtbar erscheint. Die Items der Schätzfrage weisen jedoch den entscheidenden Vorteil auf, dass diese Werte exaktere Schätzwerte des durchschnittlichen Spielverhaltens einer einzelnen befragten Person für einen typischen Schul- bzw. Samstag erlauben. So kann z. B. ein Kind, welches ansonsten exzessiv Computerspiele spielt, ausgerechnet an dem der Befragung vorangegangenen Tag nicht dazu gekommen sein und würde bei Verwendung der Gestern-Frage dann fälschlicherweise der Gruppe der Niespieler zugeordnet werden. Die Gestern-Frage ist auf der anderen Seite besser dazu geeignet auf der Gruppenebene exaktere Nutzungsmittelwerte zu ermitteln sowie verschiedene Tätigkeiten zueinander in Beziehung zu setzen.

\begin{tabular}{|c|c|c|c|c|c|c|c|}
\hline \multicolumn{8}{|c|}{$\begin{array}{l}\text { 56. Wie lange spielst du an einem Schultag oder an einem Samstag Computer- } \\
\text { oder Videospiele? }\end{array}$} \\
\hline Computer-I Videospiele & $\begin{array}{l}\text { bis eine } \\
\text { halbe } \\
\text { Stunde }\end{array}$ & $\begin{array}{c}1 / 2 \\
\text { bis } 1 \\
\text { Stunde }\end{array}$ & $\begin{array}{l}1 \\
\text { bis } 1 \frac{1}{2} \\
\text { Stunden }\end{array}$ & $\begin{array}{c}11 / 2 \\
\text { bis } 2 \\
\text { Stunden }\end{array}$ & $\begin{array}{c}2 \\
\text { bis } 3 \\
\text { Stunden }\end{array}$ & $\begin{array}{l}\text { mehr } \\
\text { als } 3 \\
\text { Stunden }\end{array}$ & $\begin{array}{l}\text { Ich spiele } \\
\text { nicht }\end{array}$ \\
\hline An einem Schultag & $\square$ & $\square$ & $\square$ & $\square$ & $\square$ & $\square$ & $\square$ \\
\hline An einem Samstag & $\square$ & $\square$ & $\square$ & $\square$ & $\square$ & $\square$ & $\square$ \\
\hline
\end{tabular}

Abbildung 7. „Schätzfrage“ am Beispiel der Computerspiele.

$11 \mathrm{Zu}$ berücksichtigen ist dabei, dass die Erfassung der samstäglichen Mediennutzungszeiten im Vergleich zu den sonntäglichen noch eine konservative Schätzung der Wochenendzeiten darstellt, da an Sonntagen die Mediennutzungszeiten noch höher liegen. 
Zur gleichzeitigen Berücksichtigung der Fernseh- und Computerspielzeiten an einem Schul- sowie an einem Samstag wurde jeweils ein Index wöchentlicher Mediennutzung berechnet. Hierfür wurden die Schätzfragen für das Computerspielen sowie das Fernsehen zunächst auf einen Einstundenabstand ${ }^{12}$ umcodiert und jeweils für das Fernsehen und das Computerspielen bei einer fünffachen Gewichtung des Wochentages sowie einer zweifachen Gewichtung des Samstages aufsummiert und schließlich an der Anzahl der Wochentage relativiert. ${ }^{13}$

\section{Medieninhalte}

10345

Die konsumierten Bildschirmmedieninhalte der Grundschülerinnen und Grundschüler wurden analog zur KFN-Schülerbefragung 2005 (vgl. Mößle et al., 2007) über zwei verschiedene Abfragearten erhoben. Zum einen für das Fernsehen sowie die Computerspielnutzung über die Fragen „Hast du dir schon einmal Filme angeschaut, die erst ,ab 16' oder ,ab 18' waren“ bzw. „Hast du schon einmal Computer- oder Videospiele gespielt, die erst ,ab 16' oder ,ab 18' waren“ (Lebenszeitprävalenz). Für beide Medien folgte dabei die Nachfrage „Wenn ja: Wie häufig in den letzten 4 Wochen?" mit den Antwortmöglichkeiten ,gar nicht“, „1 bis 2 mal“, „3 bis 6 mal“, „,noch häufiger“ (vgl. Abbildung 8). In den Fragebögen der vierten, fünften und sechsten Klasse hat sich unmittelbar an die Frage „Hast du schon einmal Computer- oder Videospiele gespielt, die erst ,ab 16' oder , ab 18' waren“, zusätzlich eine vertiefende Frage danach angeschlossen, mit wem diese Spiele gespielt wurden und woher sie diese Spiele bekommen haben (vgl. Abbildung 8).

120 = ,ich schaue nicht fern“, 1 = „,bis eine Stunde“, 2 = ,,eine bis zwei Stunden“, 3 = „,zwei bis drei Stunden“, 4 = ,mehr als drei Stunden“.

13

Index wöchentlicher Mediennutzung $\mathrm{TV}=\frac{5 \times \text { Wochentag }_{\mathrm{TV}}+2 \times \operatorname{Samstag}_{\mathrm{TV}}}{7}$

Index wöchentlicher Mediennutzung ${ }_{\mathrm{PC}}=\frac{5 \times \text { Wochentag }_{\mathrm{PC}}+2 \times \operatorname{Samstag}_{\mathrm{PC}}}{7}$. 


\begin{tabular}{|c|c|c|c|c|c|c|}
\hline \multicolumn{7}{|c|}{ 57. Hast du schon einmal Computer- oder Videospiele gespielt, die...? } \\
\hline & \multirow[b]{2}{*}{ Nein } & \multirow[b]{2}{*}{$\mathrm{Ja}$} & \multicolumn{4}{|c|}{$\rightarrow$ Wenn ja: Wie häufig in den letzten 4 Wochen? } \\
\hline & & & gar nicht & $\begin{array}{l}\text { 1- oder 2- } \\
\text { mal }\end{array}$ & 3- bis 6-mal & $\begin{array}{c}\text { noch } \\
\text { häufiger }\end{array}$ \\
\hline erst „ab 16“ waren? & $\square$ & $\square \rightarrow$ & $\square$ & $\square$ & $\square$ & $\square$ \\
\hline erst „ab 18“ waren? & $\square$ & $\square \rightarrow$ & $\square$ & $\square$ & $\square$ & $\square$ \\
\hline \multicolumn{7}{|c|}{$\rightarrow$ Wenn ja, mit wem hast du diese Spiele gespielt? } \\
\hline \multicolumn{2}{|c|}{$\square$ alleine } & \multicolumn{2}{|c|}{$\square$ mit Freunden/anderen Kindern } & \multicolumn{3}{|c|}{$\square$ mit Geschwistern } \\
\hline$\square$ mit meinem leiblichen $v$ & & $\square$ mit meine & iblichen Mutter & \multicolumn{3}{|c|}{$\square$ mit anderen Erwachsenen } \\
\hline \multicolumn{7}{|c|}{$\rightarrow$ Wenn ja, wie bist du an diese Spiele gekommen? } \\
\hline \multirow{2}{*}{\multicolumn{2}{|c|}{$\square$ durch Freunde/andere Kinder }} & \multicolumn{2}{|c|}{$\square$ durch Geschwister } & \multicolumn{3}{|c|}{$\square$ selbst besorgt } \\
\hline & & \multicolumn{2}{|c|}{$\square$ durch meine leibliche Mutter } & \multicolumn{3}{|c|}{$\square$ durch andere Erwachsene } \\
\hline
\end{tabular}

Abbildung 8. Konsum altersinadäquater Medien am Beispiel der Computerspiele.

Im Bereich der Computerspiele wurden die konsumierten Inhalte darüber hinaus wiederum analog zur KFN-Schülerbefragung 2005 (vgl. Mößle et al., 2007) $\mathrm{zu}$ allen Messzeitpunkten noch expliziter über die Frage „Welche beiden (3. Klasse) bzw. drei (4., 5. und 6. Klasse) Computer- oder Videospiele spielst du momentan am liebsten?“ erhoben (vgl. Abbildung 9). Bei der Vielzahl an Fernsehprogrammen und Filmen auf DVD oder Video war eine Abfrage vergleichbar mit der zu den Computerspielen aus forschungstechnischen Gründen leider nicht möglich.

\section{Welche drei Computer- oder Videospiele spielst du momentan am liebsten?}

Gar keine, denn ich spiele nie solche Spiele.

1.

2.

3.

Abbildung 9. Erfassung der Lieblingsspiele.

Alle von den Schülerinnen und Schülern genannten Computerspiele wurden dann nach ihrer von der Unterhaltungssoftware Selbstkontrolle (USK) festgelegten Alterseinstufung codiert: USK $0=$ Freigegeben ohne Altersbeschränkung; USK 6 = Freigegeben ab 6 Jahren; USK $12=$ Freigegeben ab 12 Jahren; USK $16=$ Freigegeben ab 16 Jahren; USK $18=$ keine Jugendfreigabe, keine Kennzeichnung, Indizierung. Die Vergabe einer Freigabe „ab 16 Jahren“ bzw. 
„keine Jugendfreigabe (ab 18 Jahren)“ lässt mit großer Sicherheit auf einen erheblichen Gewaltgehalt eines Spieles schließen, der für jüngere Spieler als „,entwicklungsbeeinträchtigend“ eingeschätzt wird. Weigert sich die USK (zumeist wegen zu extremer Gewaltdarstellungen), eine Kennzeichnung zu vergeben, wird ein Spiel mit einiger Sicherheit von der Bundesprüfstelle für jugendgefährdende Medien (BPjM) als ,,jugendgefährdend“ oder ,schwer jugendgefährdend“ indiziert, also vom öffentlichen Verkauf ausgeschlossen. Auch solche Spiele wurden somit in unserer Codierung erfasst.

In den Fragebögen der fünften und sechsten Klasse wurden neben den Spieletiteln auch die allgemeinen Genrevorlieben der Kinder über die Frage „Wie häufig spielst du die folgenden Arten von Computer- oder Videospielen“" erhoben (vgl. Abbildung 10). Die Variable Genrepräferenz wurde dann wie folgt gebildet: Schüler, die auf diese Frage „nie“ oder ,selten“ ankreuzten haben keine Präferenz für dieses Genre. Schüler, die „sehr oft“ ankreuzten haben eine Präferenz. Haben die Schüler „manchmal“ oder „oft“ angekreuzt besteht eine Genrepräferenz, wenn kein weiteres Genre öfter gespielt wird. Da Mehrfachnennungen möglich waren, wurde zur Bestimmung einer relativen Genrevorliebe die Genrepräferenz eines jeden Genres zudem an der Gesamtzahl der präferierten Genres relativiert. ${ }^{14}$

\begin{tabular}{llllll}
\hline 54. Wie häufig spielst du die folgenden Arten von Computer- oder Videospielen? \\
Wenn du gar nicht spielst, dann kreuze überall „nie“ an.
\end{tabular}

Abbildung 10. Erfassung der Computerspielgenres.

14 Relative Genrevorliebe $\mathrm{Genre} \mathrm{X}=\frac{1}{\text { Gesamtzahl der Genrepräferenzen }}$ 
Analog zur offenen Abfrage der Spieletitel wurde in den Schülerfragebögen der fünften und sechsten Klasse auch die Internetnutzung mit der Frage „Welche drei Internetseiten nutzt du momentan am liebsten?" differenzierter erhoben.

\section{Medienerziehung}

10345

In allen fünf Befragungswellen wurde in den Schülerfragebögen die durch die Kinder wahrgenommene elterliche Medienerziehung getrennt für die Bereiche Fernsehen und Computerspiele abgefragt. Zusätzlich wurde in der fünften und sechsten Klasse die elterliche Medienerziehung für das Internet in die Befragung mit aufgenommen. Die hierzu verwendete „Skala regulativen Monitorings“ wurde erstmalig von Mößle und Kollegen (Mößle et al., 2007) in der KFN-Schülerbefragung 2005 in der vierten Klasse eingesetzt und bildet eine restriktive elterliche Begleitung bei der Mediennutzung ab. Bei der restriktiven Begleitung (restrictive mediation) werden klare inhaltliche und zeitliche Regeln gesetzt und zum Teil auch Verbote ausgesprochen. Im Vergleich dazu kann eine aktive/instruktive Begleitung (active mediation) unterschieden werden, die auch eine kritische Erklärung bestimmter Inhalte und Handlungsweisen beinhaltet, bzw. das social coviewing, bei dem Eltern gewohnheitsmäßig mit ihren Kindern gemeinsam Medien konsumieren und den Inhalt kritisch beleuchten, (vgl. Böcking, 2006; Nathanson, 1999; Valkenburg, Krcmar, Peeters \& Marseille, 1999; Warren, Gerke \& Kelly, 2002)..${ }^{15}$ Zur Bildung einer Skala des wahrgenommen regulativen elterlichen Monitorings im Bereich Medien wurde aus den Kinderangaben jeweils für das Fernsehen, das Computer- oder Videospielen sowie die Internetnutzung eine Summe gebildet, die sich aus den Bereichen inhaltliche Regeln, Monitoring (d. h. elterliches Interesse für das Medienverhalten der Kinder) und zeitliche Regeln zusammensetzt.

Für die Fernsehnutzung wurden dabei nachfolgende Items verwendet: „Bei uns zu Hause gibt es klare Regeln welche Sendungen ich sehen darf und welche nicht“ (Inhalte), „Meine Eltern wollen wissen, was ich mir im Fernsehen ansehe“ (Monitoring), „Wir haben klare zeitliche Regeln, wie lange ich fernsehen darf" (Zeit). Für das Computerspielen gingen folgende Items in den gebildeten Index ein: „Bei uns gibt es klare Regelungen welche Spiele ich spielen darf und welche nicht“ (Inhalte), „Meine Eltern wollen wissen, welche Video- und Computerspiele ich spiele“ (Monitoring), „Wir haben klare zeitliche Regeln, wie lang ich Computer spielen darf" (Zeit). Und für das Internet waren es schließlich folgende drei Items: „Bei uns zu Hause gibt es klare Regelungen, welche Dinge ich im

15 Böcking (2007) zeigte z. B. für die Schweiz eine vorherrschend restriktive Begleitung, wobei diese mit dem Alter abnimmt (vgl. Warren et al., 2002). Eine restriktive elterliche Begleitung kann auch dazu führen, dass Kinder Gewalt in den Medien als weniger wichtig erachten, Gewaltinhalte weniger stark bevorzugen und eine insgesamt geringere Gewaltneigung haben (vgl. Nathanson, 1999). 
Tabelle 13. Skalen- und Itemkennwerte der Skala regulatives Monitoring nach Bildschirmmedium und Messzeitpunkt (Kinderangaben).

\begin{tabular}{|c|c|c|c|c|c|c|c|c|c|}
\hline \multicolumn{2}{|c|}{$\begin{array}{l}\text { 3. Klasse } \\
\text { HJ }\end{array}$} & \multicolumn{2}{|c|}{ 3. Klasse } & \multicolumn{2}{|c|}{ 4. Klasse } & \multicolumn{2}{|c|}{ 5. Klasse } & \multicolumn{2}{|c|}{ 6. Klasse } \\
\hline $\begin{array}{c}M \\
(S D)\end{array}$ & $\boldsymbol{r}_{i t}$ & $\begin{array}{c}M \\
(S D)\end{array}$ & $\boldsymbol{r}_{i t}$ & $\begin{array}{c}M \\
(S D)\end{array}$ & $\boldsymbol{r}_{i t}$ & $\begin{array}{c}M \\
(S D)\end{array}$ & $r_{i t}$ & $\begin{array}{c}M \\
(S D)\end{array}$ & $\boldsymbol{r}_{i t}$ \\
\hline
\end{tabular}

\section{Fernsehnutzung}

Bei uns zu Hause gibt es klare Regeln welche Sendungen ich sehen darf und welche nicht.

Meine Eltern wollen wissen, was ich mir im Fernsehen ansehe.

2.35

(1.2) $49 \quad 2.41$

2.20

(1.2)

Wir haben klare zeitliche Regeln, wie lange ich fernsehen darf.

2.45

(1.2)

$M(S D)$

$7.01(2.74)$

$43 \quad 2.36$

$.43 \quad(1.1)$

43

2.36

2.36

$\begin{array}{lll}.49 & 2.41 & .59\end{array}$

\section{Cronbachs $\alpha$} .64

59

2.47

(1.1)

.61

2.39

(1.1)

.59

2.51

(1.2)

.68

2.47

.58

2.37

2.34

(1.1) $\quad .57 \quad(1.3)$

.71

$\begin{array}{lll}2.23 & 53 & 2.50\end{array}$

.54

2.3

.57

.56

\begin{tabular}{lllllllll} 
Cronbachs $\alpha$ & .64 & .75 & .76 & .74 & .80 \\
\hline
\end{tabular}

\section{Computerspielnutzung}

Bei uns gibt es klare Regelungen welche Spiele ich spielen darf und welche nicht.

\subsection{8}

(1.2)

Meine Eltern wollen wissen, welche Video- und Computerspiele ich spiele.

Wir haben klare zeitliche Regeln, wie lang ich Computer spielen darf.

$\begin{array}{llllllllll}2.10 & .64 & 2.21 & .66 & 2.44 & .71 & 2.78 & .69 & 2.76 & \\ (1.3) & & (1.3) & .68 & (1.3) & .71 & (1.2) & .6 & (1.2) & .78\end{array}$

$\begin{array}{llllllllll}2.40 & .54 & 2.40 & .55 & 2.48 & .57 & 2.40 & .57 & 2.54 & \\ (1.2) & & (1.2) & & (1.2) & & & (1.2) & & \end{array}$

\begin{tabular}{rccccc}
$M(S D)$ & $6.78(3.06)$ & $6.94(3.08)$ & $7.50(3.12)$ & $8.31(3.01)$ & $8.07(3.09)$ \\
Cronbachs $\alpha$ & .76 & .78 & .80 & .80 & .86 \\
\hline
\end{tabular}

\section{Internetnutzung}

Bei uns zu Hause gibt es klare

Regelungen, welche Dinge ich im Internet tun darf und welche

nicht.

Meine Eltern wollen wissen, was ich im Internet mache.

Wir haben klare Regeln, wie lang ich das Internet nutzen darf.

\section{Cronbachs $\alpha$}

Anmerkung. $r_{i t}=$ Trennschärfe (Part-Whole-Korrektur). Skalenwerte von 1 bis 4 (,nie“, „manchmal“" „meistens“, ,immer"). 
Internet tun darf und welche nicht“ (Inhalte), „Meine Eltern wollen wissen, was ich im Internet mache“ (Monitoring), „Wir haben klare Regeln, wie lang ich das Internet nutzen darf" (Zeit). Als Antwortmöglichkeiten standen den Kindern für alle drei Bereiche im Fragebogen die vier Alternativen ,immer“ (4), ,meistens“ (3), „manchmal“ (2) und „nie“ (1) zur Auswahl. Mit einem Cronbachs $\alpha$ zwischen $\alpha_{3 . \text { Klasse } H J}=.64$ und $\alpha_{6 . \text { Klasse }}=.80$ für die Fernsehnutzung, zwischen $\alpha_{3 \text {. Klas- }}$ se $H_{J}=.76$ und $\alpha_{6 . \text { Klasse }}=.86$ für die Computerspielnutzung sowie einem Cronbachs $\alpha_{5 . \text { Klasse }}=.78$ und Cronbachs $\alpha_{6 . \text { Klasse }}=.82$ für die Internetnutzung ist (mit Ausnahme der Fernsehnutzung in der dritten Klasse) bei insgesamt jeweils drei Items eine befriedigende bis gute interne Konsistenz der Skala gegeben (Skalenund Itemkennwerte siehe Tabelle 13).

Zur vereinfachten Darstellung wurde die Skala regulatives Monitoring (Eltern und Kinderangaben) für die unterschiedlichen Bildschirmmedien nach der folgenden Vorschrift kategorisiert: 3-4 Punkte = kein regulatives Monitoring; 5-7 Punkte $=$ geringes regulatives Monitoring; 8-10 Punkte $=$ mittleres regulatives Monitoring; 11-12 Punkte $=$ hohes regulatives Monitoring. Die Skalenlogik folgt dabei dem Gedanken, dass Kinder mit keinem regulativen Monitoring maximal nur einem Item mit „manchmal“ zustimmen konnten. Kinder mit hohem regulativem Monitoring haben auf der anderen Seite mindestens zwei Items mit „immer" zugestimmt. Entsprechend haben Kinder mit geringem regulativem Monitoring im Mittel $( \pm 1)$ mit ,,manchmal“ und Kinder mit mittlerem regulativen Monitoring im Mittel $( \pm 1)$ mit „,meistens“ zugestimmt.

\section{Elterliches Erziehungs- und Unterstützungsverhalten}

10845

Das elterliche Erziehungs- und Unterstützungsverhalten wurde in den Fragebögen der dritten bis sechsten Klasse vergleichbar zur KFN-Schülerbefragung 2000 (Skalen positive elterliche Zuwendung und elterliche Supervision, hier retrospektiv für die Zeit vor dem 12. Lebensjahr; Wilmers et al., 2002) und KFNSchülerbefragung 2005 (Mößle et al., 2007) erhoben. Angelehnt an das Verständnis elterlichen Erziehungsverhaltens nach Graf (2005) setzt sich die „Skala allgemeines elterliches Erziehungs- und Unterstützungsverhalten“ aus den Bereichen Monitoring, Beziehung, emotionale Nähe, Wertschätzung, Unterstützung und Interesse zusammen (Skalen- und Itemkennwerte siehe Tabelle 14). Folgende Items, die jeweils getrennt für die Mutter und den Vater abgefragt wurden, gingen dabei in die Skalenbildung ein: „Meine Mutter/mein Vater weiß was ich in meiner Freizeit mache“ (Monitoring/Interesse), „Meine Mutter/mein Vater ist jemand mit der/dem ich über alles reden kann“" (Beziehung/emotionale Nähe), „Meine Mutter/mein Vater hilft mir bei den Hausaufgaben, wenn ich Hilfe brauche“ (Unterstützung/Interesse), „Meine Mutter/mein Vater fragt mich, wie es in der Schule war“ (Interesse) und „Meine Mutter/mein Vater lobt mich, wenn ich 
etwas gut gemacht habe“ (Wertschätzung/Beziehung). Als Antwortmöglichkeiten standen den Kindern jeweils für die Mutter und den Vater die vier Antwortalternativen „,nie“ (1), „manchmal“ (2), „meistens“ (3) und ,immer“ (4) zur Auswahl (vgl. Tabelle 14). Zur Bildung der Skala wurden diese Antwortalternativen zunächst für den Vater und die Mutter aufsummiert. Mit einem Cronbachs $\alpha$ zwischen $\alpha_{3 \text {. Klasse } H J}=.58$ und $\alpha_{6 . \text { Klasse }}=.68$ für die Mutter sowie zwischen $\alpha_{3 \text {. Klas- }}$ $s e=.69$ und $\alpha_{5 . \text { Klasse }}=.75$ für den Vater ist bei insgesamt jeweils fünf Items eine akzeptable bis befriedigende interne Konsistenz der Skala gegeben (Item- und

Tabelle 14. Skalen- und Itemkennwerte der „Skala elterliches Erziehungs- und Unterstützungsverhalten" nach Messzeitpunkt.

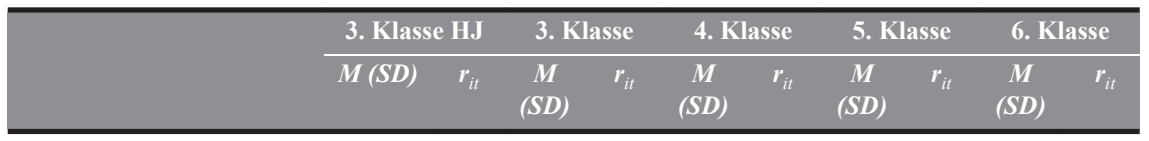

\section{Meine Mutter...}

\begin{tabular}{|c|c|c|c|c|c|c|c|c|c|c|}
\hline $\begin{array}{l}\text { weiß was ich in meiner Frei- } \\
\text { zeit mache. }\end{array}$ & $\begin{array}{l}3.06 \\
(.91)\end{array}$ & .36 & $\begin{array}{l}3.15 \\
(.81)\end{array}$ & .38 & $\begin{array}{l}3.29 \\
(.77)\end{array}$ & .48 & $\begin{array}{l}3.27 \\
(.76)\end{array}$ & .46 & $\begin{array}{l}3.20 \\
(.74)\end{array}$ & .44 \\
\hline $\begin{array}{l}\text { ist jemand mit der ich über } \\
\text { alles reden kann. }\end{array}$ & $\begin{array}{l}3.36 \\
(.87)\end{array}$ & .38 & $\begin{array}{l}3.42 \\
(.81)\end{array}$ & .43 & $\begin{array}{l}3.44 \\
(.84)\end{array}$ & .48 & $\begin{array}{l}3.39 \\
(.84)\end{array}$ & .45 & $\begin{array}{l}3.27 \\
(.89)\end{array}$ & .46 \\
\hline $\begin{array}{l}\text { hilft mir bei den Hausaufga- } \\
\text { ben, wenn ich Hilfe brauche. }\end{array}$ & $\begin{array}{c}3.25 \\
(1.00)\end{array}$ & .34 & $\begin{array}{l}3.28 \\
(.97)\end{array}$ & .33 & $\begin{array}{l}3.40 \\
(.89)\end{array}$ & .40 & $\begin{array}{l}3.30 \\
(.95)\end{array}$ & .44 & $\begin{array}{l}3.19 \\
(.98)\end{array}$ & .49 \\
\hline $\begin{array}{l}\text { fragt mich, wie es in der } \\
\text { Schule war. }\end{array}$ & $\begin{array}{l}3.38 \\
(.89)\end{array}$ & .33 & $\begin{array}{l}3.37 \\
(.86)\end{array}$ & .41 & $\begin{array}{l}3.41 \\
(.86)\end{array}$ & .46 & $\begin{array}{l}3.47 \\
(.81)\end{array}$ & .40 & $\begin{array}{l}3.40 \\
(.87)\end{array}$ & .44 \\
\hline $\begin{array}{l}\text { lobt mich, wenn ich etwas } \\
\text { gut gemacht habe. }\end{array}$ & $\begin{array}{l}3.66 \\
(.65)\end{array}$ & .31 & $\begin{array}{l}3.63 \\
(.66)\end{array}$ & .37 & $\begin{array}{l}3.67 \\
(.63)\end{array}$ & .43 & $\begin{array}{l}3.68 \\
(.61)\end{array}$ & .40 & $\begin{array}{l}3.56 \\
(.72)\end{array}$ & .43 \\
\hline$M(S D)$ & 16.60 & .73) & 16.8 & $2.51)$ & 17.2 & 2.67) & 16.99 & $2.67)$ & 16.5 & $.83)$ \\
\hline
\end{tabular}

$\begin{array}{lllllll}\text { Cronbachs } \alpha & .58 & .58 & .67 & .64 & .68\end{array}$

\section{Mein Vater...}

weiß was ich in meiner Frei- $\quad 2.62$

zeit mache.

ist jemand mit dem ich über alles reden kann.

hilft mir bei den Hausaufgaben, wenn ich Hilfe brauche. fragt mich, wie es in der Schule war.

lobt mich, wenn ich etwas gut gemacht habe.

$\begin{array}{cccccccccc}2.62 & .43 & 2.69 & .41 & 2.76 & .54 & 2.81 & .54 & 2.71 & .53 \\ (1.02) & & (.95) & & (.96) & & (.90) & & (.92) & \\ 2.99 & .42 & 3.12 & & 3.06 & .54 & 3.01 & .56 & 2.80 & .53 \\ (1.02) & & (.98) & .50 & (1.0) & .5 & (1.0) & & (1.1) & \\ 2.73 & .48 & 2.90 & & 3.16 & & 3.05 & .54 & 3.03 & .58 \\ (1.12) & & (1.1) & .49 & (1.0) & .53 & (1.1) & & (1.1) & \\ 2.94 & .51 & 2.96 & & 2.98 & & 3.05 & .50 & 2.96 & .57 \\ (1.12) & & (1.1) & .48 & (1.1) & .49 & (1.0) & & (1.0) & \\ 3.51 & .48 & 3.54 & & 3.56 & & 3.53 & & 3.45 & .56 \\ (.80) & & (.76) & .49 & (.74) & .49 & (.77) & .54 & (.86) & .56 \\ 14.59(3.54) & 15.12(3.28) & 15.45(3.48) & 15.37 & (3.45) & 14.93 & (3.62)\end{array}$
.69

.75

.75

.71

$\begin{array}{llllll}\text { Gesamtskala M (SD) } & 15.69(2.81) & 16.06(2.58) & 16.42(2.74) & 16.27(2.73) & 15.81 \\ (2.84)\end{array}$

Anmerkung. $r_{i t}=$ Trennschärfe (Part-Whole-Korrektur). Skalenwerte von 1 bis 4 (,nie“, „,manchmal" „,meistens", ,immer"). 
Skalenkennwerte siehe Tabelle 14). Anschließend wurde eine Mittelwertskala ${ }^{16}$ aus dem väterlichen und mütterlichen Erziehungs- und Unterstützungsverhalten gebildet. Mit Hilfe der daraus resultierenden Skala wurden die Schülerinnen und Schüler schließlich in drei Kategorien unterschiedlichen elterlichen Erziehungsund Unterstützungsverhaltens eingeteilt: geringe elterliche Zuwendung (5-10), mittlere elterliche Zuwendung (11-15), hohe elterliche Zuwendung (16-20).

\section{Erlebte Elterngewalt}

00845

Abbildung 11 zeigt die Items, mit denen sowohl in der vierten als auch in der fünften und sechsten Klasse häusliche Gewalt erhoben wurde. Die „Skala erlebte Elterngewalt" wurde bereits in den KFN-Schülerbefragungen 2000 (Wilmers et al., 2002) , 2005 (Baier, Pfeiffer, Windzio \& Rabold, 2006) und 2007/2008 (Baier, Pfeiffer, Rabold \& Kappes, 2010) eingesetzt und stellt eine deutsche Adaptation der Conflict Tactic (CT) scales von Straus (1979) dar. Aufgrund der Antworten für Mutter und Vater wurde folgendermaßen kategorisiert: Berichten die Kinder davon, dass ihnen von Mutter oder Vater innerhalb der letzten vier Wochen vor der Befragung mindestens einmal „eine runtergehauen wurde“, dass von ihrer Mutter oder ihrem Vater ,mit einem Gegenstand nach ihnen geworfen wurde“, oder dass sie von ihrer Mutter oder ihrem Vater ,hart angepackt oder gestoßen wurden“, wird dies als schwere Züchtigung gewertet. ${ }^{17}$ Berichten die Kinder davon, dass ihr Vater oder ihre Mutter sie „mit der Faust geschlagen oder getreten haben“ oder dass ihr Vater oder ihre Mutter sie „geprügelt haben bzw. sie zusammengeschlagen haben“, wird dies als Misshandlung durch ihre Eltern gewertet. ${ }^{18}$ Als Häufigkeitsindikator wurde das Item herangezogen, bei dem die höchste Zahl eingetragen wurde.

16 Bei Vorliegen eines Missing-Wertes (Mutter oder Vater) wurde jeweils die vorhandene Skala (Vater oder Mutter) gewertet.

17 Gewertet wurde die höchste Nennung aus Angaben der Mutter und des Vater (MAX.1).

18 Gewertet wurde die höchste Nennung aus Angaben der Mutter und des Vater (MAX.1). 


\begin{tabular}{|c|c|c|c|c|c|c|c|c|}
\hline \multirow[b]{2}{*}{$\begin{array}{l}\text { Meine Mutter/mein Vater } \\
\text { hat... }\end{array}$} & \multicolumn{4}{|c|}{ Mutter } & \multicolumn{4}{|c|}{ Vater } \\
\hline & nie & $\begin{array}{l}\text { 1- oder } \\
\text { 2-mal }\end{array}$ & $\begin{array}{l}\text { 3- bis 6- } \\
\text { mal }\end{array}$ & $\begin{array}{l}\text { noch } \\
\text { häufiger }\end{array}$ & nie & $\begin{array}{l}\text { 1- oder } \\
\text { 2-mal }\end{array}$ & $\begin{array}{c}\text { 3- bis 6- } \\
\text { mal }\end{array}$ & $\begin{array}{c}\text { noch } \\
\text { hăufiger }\end{array}$ \\
\hline mir eine runtergehauen. & $\square$ & $\square$ & $\square$ & $\square$ & $\square$ & $\square$ & $\square$ & $\square$ \\
\hline $\begin{array}{l}\text { mit einem Gegenstand nach } \\
\text { mir geworfen. }\end{array}$ & $\square$ & $\square$ & $\square$ & $\square$ & $\square$ & $\square$ & $\square$ & $\square$ \\
\hline $\begin{array}{l}\text { mich hart angepackt oder } \\
\text { gestoßen. }\end{array}$ & $\square$ & $\square$ & $\square$ & $\square$ & $\square$ & $\square$ & $\square$ & $\square$ \\
\hline $\begin{array}{l}\text { mich mit der Faust geschlagen } \\
\text { oder mich getreten. }\end{array}$ & $\square$ & $\square$ & $\square$ & $\square$ & $\square$ & $\square$ & $\square$ & $\square$ \\
\hline $\begin{array}{l}\text { mich geprügelt bzw. mich } \\
\text { zusammengeschlagen. }\end{array}$ & $\square$ & $\square$ & $\square$ & $\square$ & $\square$ & $\square$ & $\square$ & $\square$ \\
\hline
\end{tabular}

Abbildung 11. Erfassung erlebter Elterngewalt.

\section{Devianz und Delinquentes Verhalten}

Im Berliner Längsschnitt Medien wurden erstmalig in der vierten Klasse abweichendes Verhalten, Mobbing und psychische Gewalt in der Schule vergleichbar zu den KFN-Schülerbefragungen 2005 (Baier et al., 2006) und 2007/2008 (Baier et al., 2010) sowie die verschiedenen „Deliktbereiche“ delinquenten Verhaltens außerhalb der Schule vergleichbar zu den KFN-Schülerbefragungen 2000 (Wilmers et al., 2002; vgl. Delinquenzbelastungsskala, DBS-Skala; Lösel, 1975), 2005 (Baier et al., 2006) und 2007/2008 (Baier et al., 2010) abgefragt (vgl. Abbildung 12 und Abbildung 13). Abweichendes Verhalten wurde für die Bereiche Schulabsentismus, Alkohol- und Zigarettenkonsum sowie das Betrachten „schlimmer“ Filme auf dem Handy erfragt. ${ }^{19}$ Delinquentes Verhalten wurde aus der Täter- und Opferperspektive erhoben. So wurden die Schülerinnen und Schüler zum einen gefragt, ob sie innerhalb der letzten 12 Monate (Delinquentes Verhalten außerhalb der Schule) bzw. 4 Wochen (Mobbing und Gewalt in der Schule) bestimmte Dinge getan haben (Delinquenz-Täterperspektive: ${ }^{20}$ „Einem anderen Kind absichtlich so sehr wehgetan, dass es geweint hat oder verletzt war",

19 „Hast du schon jemals folgende Dinge getan? Wenn ja: Wie oft in den letzten 12 Monaten?“ Wurde ,ja“" und eine Zahl für die letzten 12 Monate eingetragen, wurde dies als ein Ereignis gewertet. Folgende Bereiche wurden abgefragt: „Mehr als einen Schluck Alkohol getrunken“, „Eine Zigarette geraucht“, „Einen ganzen Tag die Schule geschwänzt", „Einzelne Stunden die Schule geschwänzt“", „Auf dem Handy Filme oder Bilder angeschaut, auf denen etwas Schlimmes zu sehen war".

20 „Hast du schon jemals folgende Dinge getan? Wenn ja: Wie oft in den letzten 12 Monaten?" Wurde ,ja“" und eine Zahl für die letzten 12 Monate eingetragen, wurde dies als ein Ereignis gewertet. 
„Einem anderen Kind gedroht, damit es dir etwas gibt“, „In einem Kaufhaus oder Geschäft etwas gestohlen“, „Absichtlich Fenster, Telefonzellen, Straßenlampen oder ähnliche Dinge beschädigt“, „Jemandem eine Sache oder Geld gestohlen“, „Gezündelt oder etwas in Brand gesteckt"; Schulmobbing-Täterperspektive: ${ }^{21}$ „Ich habe einen Schüler gehänselt oder hässliche Dinge über ihn gesagt", „Ich habe einen anderen Schüler wie Luft behandelt und absichtlich nicht mehr beachtet", „Ich habe Gerüchte über einen anderen Schüler verbreitet, die nicht wahr gewesen sind", „Ich war dagegen, dass ein anderes Kind mit mir und meinen Freunden zusammen in der Pause spielt"; Schulgewalt-Täterperspekti$v e:{ }^{22}$ "Ich habe einen Schüler geschlagen oder getreten, und zwar nicht aus Spaß", „Ich habe Sachen von einem Schüler mit Absicht kaputtgemacht“, „Ich habe eine anderes Kind gezwungen, etwas zu tun, was es nicht wollte").

\begin{tabular}{|c|c|c|c|c|}
\hline & $\begin{array}{c}\text { gar } \\
\text { nicht }\end{array}$ & $\begin{array}{l}\text { 1- oder } \\
2 \text {-mal }\end{array}$ & $\begin{array}{l}3 \text { - bis } \\
6 \text {-mal }\end{array}$ & $\begin{array}{l}\text { noch } \\
\text { häufiger }\end{array}$ \\
\hline $\begin{array}{l}\text { Ich habe einen Schüler geschlagen oder getreten, } \\
\text { und zwar nicht aus Spaß. }\end{array}$ & $\square$ & $\square$ & $\square$ & $\square$ \\
\hline $\begin{array}{l}\text { Ich habe einen Schüler gehänselt oder hässliche } \\
\text { Dinge über inn gesagt. }\end{array}$ & $\square$ & $\square$ & $\square$ & $\square$ \\
\hline $\begin{array}{l}\text { Ich habe einen anderen Schüler wie Luft behandelt } \\
\text { und absichtlich nicht mehr beachtet. }\end{array}$ & $\square$ & $\square$ & $\square$ & $\square$ \\
\hline $\begin{array}{l}\text { Ich habe Gerüchte über einen anderen Schüler } \\
\text { verbreitet, die nicht wahr gewesen sind. }\end{array}$ & $\square$ & $\square$ & $\square$ & $\square$ \\
\hline $\begin{array}{l}\text { Ich war dagegen, dass ein anderes Kind mit mir und } \\
\text { meinen Freunden zusammen in der Pause spielt. }\end{array}$ & $\square$ & $\square$ & $\square$ & $\square$ \\
\hline $\begin{array}{l}\text { Ich habe Sachen von einem Schüler mit Absicht } \\
\text { kaputtgemacht. }\end{array}$ & $\square$ & $\square$ & $\square$ & $\square$ \\
\hline $\begin{array}{l}\text { Ich habe ein anderes Kind gezwungen, etwas zu } \\
\text { tun, was es nicht wollte. }\end{array}$ & $\square$ & $\square$ & $\square$ & $\square$ \\
\hline
\end{tabular}

Abbildung 12. Erfassung Schulgewalt und Schulmobbing Täterperspektive.

21 „Wie oft hast du in den letzten 4 Wochen so etwas in der Schule gemacht? (,gar nicht“, „1- oder 2-mal", ,3- bis 6-mal", „,noch häufiger").

22 "Wie oft hast du in den letzten 4 Wochen so etwas in der Schule gemacht? (,gar nicht", „1- oder 2-mal“", „3- bis 6-mal“, „,noch häufiger"). 


\begin{tabular}{|c|c|c|c|c|}
\hline \multicolumn{5}{|c|}{$\begin{array}{l}\text { 66. Jetzt geht es darum, ob du schon einmal folgende Dinge getan hast? } \\
\text { Trage bitte „, } 0^{4} \text { ein, wenn du das im letzten Jahr nicht getan hast. }\end{array}$} \\
\hline & \multicolumn{2}{|c|}{$\begin{array}{l}\text { Hast du das schon } \\
\text { jemals getan? }\end{array}$} & \multirow[t]{2}{*}{$\rightarrow$} & \multirow{2}{*}{$\begin{array}{l}\text { Wenn ja: Wie oft } \\
\text { in den letzten } 12 \\
\text { Monaten? }\end{array}$} \\
\hline & Nein & $\mathrm{Ja}$ & & \\
\hline Mehr als einen Schluck Alkohol getrunken. & $\square$ & $\square$ & $\rightarrow$ & - mal \\
\hline $\begin{array}{l}\text { Einem anderen Kind absichtlich so sehr wehgetan, } \\
\text { dass es geweint hat oder verletzt war. }\end{array}$ & $\square$ & $\square$ & $\rightarrow$ & mal \\
\hline Eine Zigarette geraucht. & $\square$ & $\square$ & $\rightarrow$ & mal \\
\hline Einem anderen Kind gedroht, damit es mir etwas gibt. & $\square$ & $\square$ & $\rightarrow$ & mal \\
\hline In einem Kaufhaus oder Geschäft etwas gestohlen. & $\square$ & $\square$ & $\rightarrow$ & - mal \\
\hline $\begin{array}{l}\text { Absichtlich Fenster, Telefonzellen, Straßenlampen } \\
\text { oder ähnliche Dinge beschädigt. }\end{array}$ & $\square$ & $\square$ & $\rightarrow$ & _ mal \\
\hline Jemandem eine Sache oder Geld gestohlen. & $\square$ & $\square$ & $\rightarrow$ & - mal \\
\hline Gezündelt oder etwas in Brand gesteckt. & $\square$ & $\square$ & $\rightarrow$ & mal \\
\hline $\begin{array}{l}\text { Auf dem Handy Filme oder Bilder angeschaut, auf } \\
\text { denen etwas Schlimmes zu sehen war. }\end{array}$ & $\square$ & $\square$ & $\rightarrow$ & mal \\
\hline Einen ganzen Tag die Schule geschwänzt. & $\square$ & $\square$ & $\rightarrow$ & mal \\
\hline Einzelne Stunden die Schule geschwänzt. & $\square$ & $\square$ & $\rightarrow$ & mal \\
\hline
\end{tabular}

Abbildung 13. Erfassung abweichendes Verhalten und Delinquenz Täterperspektive.

Für die Delinquenz außerhalb der Schule wurden die Items „In einem Kaufhaus oder Geschäft etwas gestohlen“ und „Jemandem eine Sache oder Geld gestohlen“ zu Diebstahl, ${ }^{23}$ die Items „Absichtlich Fenster, Telefonzellen, Straßenlampen oder ähnliche Dinge beschädigt“ und „Gezündelt oder etwas in Brand gesteckt“ zu Sachbeschädigung ${ }^{24}$ sowie die Items „Einem anderen Kind absichtlich so sehr wehgetan, dass es geweint hat oder verletzt war“ und „Einem anderen Kind gedroht, damit es dir etwas gibt“ zu Verletzen/Drohen ${ }^{25}$ zusammengefasst. Als Gewaltdelinquenz wurde schließlich gewertet, wenn ein Kind in einem der beiden Bereiche Sachbeschädigung oder Verletzen/Drohen innerhalb der letzten 12 Monate auffällig war. Aus den beiden Bereichen Schulgewalt und Gewaltdelinquenz wurde schließlich eine Skala mittlerer Gewaltprävalenz gebildet,

23 Gewertet wurde die höchste Nennung (MAX.1).

24 Gewertet wurde die höchste Nennung (MAX.1).

25 Gewertet wurde die höchste Nennung (MAX.1). 
die Gewaltdelinquenz innerhalb und außerhalb der Schule zu gleichen Anteilen berücksichtigt. $^{26}$

\section{In der Schule oder auf dem Schulweg gibt es auch manchmal Ärger}

\begin{tabular}{|c|c|c|c|c|}
\hline & $\begin{array}{c}\text { gar } \\
\text { nicht }\end{array}$ & $\begin{array}{l}\text { 1- oder } \\
2 \text {-mal }\end{array}$ & $\begin{array}{l}3 \text { - bis } \\
6 \text {-mal }\end{array}$ & $\begin{array}{l}\text { noch } \\
\text { häufiger }\end{array}$ \\
\hline $\begin{array}{l}\text { Ich wurde von Schülern geschlagen oder getreten, } \\
\text { und zwar nicht aus Spaß. }\end{array}$ & $\square$ & $\square$ & $\square$ & $\square$ \\
\hline $\begin{array}{l}\text { Schüler haben mich gehänselt oder hässliche Dinge } \\
\text { über mich gesagt. }\end{array}$ & $\square$ & $\square$ & $\square$ & $\square$ \\
\hline $\begin{array}{l}\text { Andere Schüler haben mich wie Luft behandelt und } \\
\text { absichtlich nicht mehr beachtet. }\end{array}$ & $\square$ & $\square$ & $\square$ & $\square$ \\
\hline $\begin{array}{l}\text { Andere Schüler haben Gerüchte über mich } \\
\text { verbreitet, die nicht wahr gewesen sind. }\end{array}$ & $\square$ & $\square$ & $\square$ & $\square$ \\
\hline $\begin{array}{l}\text { Ich durfte in der Pause nicht bei anderen Kindern } \\
\text { mitspielen. }\end{array}$ & $\square$ & $\square$ & $\square$ & $\square$ \\
\hline $\begin{array}{l}\text { Schüler haben mit Absicht meine Sachen } \\
\text { kaputtgemacht. }\end{array}$ & $\square$ & $\square$ & $\square$ & $\square$ \\
\hline $\begin{array}{l}\text { Schüler haben mich gezwungen, etwas zu tun, was } \\
\text { ich nicht wollte. }\end{array}$ & $\square$ & $\square$ & $\square$ & $\square$ \\
\hline
\end{tabular}

Abbildung 14. Erfassung Schulgewalt und Schulmobbing Opferperspektive.

\begin{tabular}{|c|c|c|c|c|}
\hline \multicolumn{5}{|c|}{$\begin{array}{l}\text { 65. Ist dir jemals eines der folgenden Dinge zugestoßen? } \\
\text { Trage bitte "O“ ein, wenn es dir im letzten Jahr nicht passiert ist. }\end{array}$} \\
\hline & Nein & $\mathrm{Ja}$ & $\rightarrow$ & $\begin{array}{l}\text { Wenn ja: Wie oft } \\
\text { in den letzten } 12 \\
\text { Monaten? }\end{array}$ \\
\hline $\begin{array}{l}\text { Mir wurde etwas gestohlen. } \\
\text { z.B. Geld oder eine Sache }\end{array}$ & $\square$ & $\square$ & $\rightarrow$ & - mal \\
\hline $\begin{array}{l}\text { Mein Eigentum wurde absichtlich beschädigt. } \\
\text { z.B. Schultasche, Handy, Jacke }\end{array}$ & $\square$ & $\square$ & $\rightarrow$ & mal \\
\hline $\begin{array}{l}\text { Mir wurde mit Gewalt oder unter Androhung von Gewalt } \\
\text { etwas weggenommen. } \\
\text { z.B. Raub von Geld, Handy, Jacke }\end{array}$ & $\square$ & $\square$ & $\rightarrow$ & mal \\
\hline $\begin{array}{l}\text { Ich wurde körperlich verletzt. } \\
\text { z.B. durch Schläge, Tritte, Angriff mit Waffe }\end{array}$ & $\square$ & $\square$ & $\rightarrow$ & $\mathrm{mal}$ \\
\hline
\end{tabular}

Abbildung 15. Erfassung abweichendes Verhalten und Delinquenz Opferperspektive.

26 Mittlere Gewaltprävalenz = Mittelwert aus Gewaltdelinquenz und Schulgewalt. Gewaltdelinquenz (Summe (Ja/Nein): „Einem anderen Kind absichtlich so sehr weh getan, dass es geweint hat oder verletzt war", „Einem andern Kind gedroht, damit es dir etwas gibt", „Absichtlich Fenster, Telefonzellen. Straßenlampen oder ähnliche Dinge beschädigt“, „Gezündelt oder etwas in Brand gesteckt"), Schulgewalt (Summe (Ja/Nein): „Ich habe einen Schüler geschlagen oder getreten, und zwar nicht aus Spaß“, „Ich habe Sachen von einem Schüler mit Absicht kaputt gemacht“, „Ich habe ein anderes Kind gezwungen, etwas zu tun, was es nicht wollte"). 
Zum anderen wurden die Schülerinnen und Schüler vor der Befragung aus der Täterperspektive gebeten darüber Auskunft zu geben, ob sie innerhalb der letzten 12 Monate (Delinquentes Verhalten außerhalb der Schule - Raub, Sachbeschädigung, Diebstahl und Körperverletzung; nur fünfte und sechste Klasse) bzw. 4 Wochen (Mobbing und Gewalt in der Schule) Opfer dieser Dinge waren (vgl. Abbildung 14 und Abbildung 15; Delinquenz-Viktimisierung: ${ }^{27}$ „Mir wurde etwas gestohlen, z. B. Geld oder eine Sache“, „Mein Eigentum wurde absichtlich beschädigt, z. B. Schultasche, Handy, Jacke“, „Mir wurde mit Gewalt oder unter Androhung von Gewalt etwas weggenommen, z. B. Raub von Geld, Handy, Jacke“, „Ich wurde körperlich verletzt, z. B. durch Schläge, Tritte, Angriff mit Waffe“; Schulmobbing-Viktimisierung: ${ }^{28}$ „Schüler haben mich gehänselt oder hässliche Dinge über mich gesagt", „Andere Schüler haben mich wie Luft behandelt und absichtlich nicht mehr beachtet", „Andere Schüler haben Gerüchte über mich verbreitet, die nicht wahr gewesen sind", „Ich durfte in der Pause nicht bei anderen Kindern mitspielen"; Schulgewalt-Viktimisierung: ${ }^{29}$ „Ich wurde von Schülern geschlagen oder getreten, und zwar nicht aus Spaß", „Schüler haben mit Absicht meine Sachen kaputtgemacht"', „Schüler haben mich gezwungen, etwas zu tun, was ich nicht wollte").

Analog zur Skala mittlerer Gewaltprävalenz wurde hierzu eine Skala mittlerer Gewaltopfererfahrung für die fünfte und sechste Klasse gebildet. ${ }^{30}$

\section{Delinquenter Freundeskreis}

00345

Der Delinquente Freundeskreis der Schülerinnen und Schüler wurde wiederum vergleichbar zu den KFN-Schülerbefragungen 2005 (Baier et al., 2006; Mößle et al., 2007) und 2007/2008 (Baier et al., 2010) erhoben (vgl. Abbildung 16).

27 „Ist dir jemals eines der folgenden Dinge zugestoßen? Trage bitte „, 0 “ ein, wenn es dir im letzten Jahr nicht passiert ist. Wenn ja: Wie oft in den letzten 12 Monate?"Wurde ,ja" und eine Zahl für die letzten 12 Monate eingetragen, wurde dies als ein Ereignis dieser Tat gewertet.

28 „Wie oft ist dir in den letzten 4 Wochen so etwas in der Schule passiert? (,gar nicht“, „1oder 2-mal“, ,3- bis 6-mal“, ,noch häufiger").

29 „Wie oft ist dir in den letzten 4 Wochen so etwas in der Schule passiert? (,gar nicht“, „1oder 2-mal", ,,3- bis 6-mal“, ,noch häufiger").

30 Mittlere Gewaltopfererfahrung = Mittelwert aus Gewaltopfererfahrung außerhalb der Schule und Opfer von Schulgewalt. Gewaltopfererfahrung (Summe (Ja/Nein): Sachbeschädigung, Raub, Köperverletzung); Schulgewalt (Summe (Ja/Nein): „Ich wurde Schülern geschlagen oder getreten, und zwar nicht aus Spaß", ,Schüler haben mit Absicht meines Sachen kaputtgemacht“, ,Schüler haben mich gezwungen, etwas zu tun, was ich nicht wollte"). 


\begin{tabular}{|c|c|c|c|c|c|}
\hline \multicolumn{6}{|c|}{$\begin{array}{l}\text { 63. Wie viele Freund/innen kennst du, die deiner Einschätzung nach in den } \\
\text { letzten } 12 \text { Monaten Folgendes getan haben? }\end{array}$} \\
\hline $\begin{array}{l}\text { Ich kenne ... Freund/innen, die in den } \\
\text { letzten } 12 \text { Monaten... }\end{array}$ & 0 & 1 & 2 & 3 bis 5 & mehr als 5 \\
\hline $\begin{array}{l}\text { in einem Kaufhaus oder Geschäft etwas } \\
\text { gestohlen haben. }\end{array}$ & $\square$ & $\square$ & $\square$ & $\square$ & $\square$ \\
\hline $\begin{array}{l}\text { den Unterricht für eine Stunde oder einen } \\
\text { Schultag geschwänzt haben. }\end{array}$ & $\square$ & $\square$ & $\square$ & $\square$ & $\square$ \\
\hline $\begin{array}{l}\text { einem anderen Kind absichtlich so sehr weh } \\
\text { getan haben, dass es verletzt war. }\end{array}$ & $\square$ & $\square$ & $\square$ & $\square$ & $\square$ \\
\hline $\begin{array}{l}\text { eine Zigarette geraucht oder mehr als einen } \\
\text { Schluck Alkohol getrunken haben. }\end{array}$ & $\square$ & $\square$ & $\square$ & $\square$ & $\square$ \\
\hline $\begin{array}{l}\text { absichtlich Fenster, Telefonzellen, } \\
\text { Straßenlampen oder ähnliche Dinge } \\
\text { beschädigt haben. }\end{array}$ & $\square$ & $\square$ & $\square$ & $\square$ & $\square$ \\
\hline gezündelt oder etwas in Brand gesteckt haben. & $\square$ & $\square$ & $\square$ & $\square$ & $\square$ \\
\hline
\end{tabular}

Abbildung 16. Erfassung des delinquenten Freundeskreises.

Die Kinder wurden bzgl. verschiedener Delikte danach gefragt, wie viele ihrer Freunde (,0“, „1“, „2“, „,3 bis 5“, „mehr als 5“) im letzten Jahr ein solches Delikt begangen haben: Ladendiebstahl (,,in einem Kaufhaus oder Geschäft etwas gestohlen“), Schulschwänzen (,den Unterricht für eine Stunde oder einen Schultag geschwänzt"), Körperverletzung (,einem anderen Kind absichtlich so sehr weh getan, dass es verletzt war"), Rauchen/Alkohol (,eine Zigarette geraucht oder mehr als einen Schluck Alkohol getrunken“), Sachbeschädigung (,absichtlich Fenster, Telefonzellen, Straßenlampen oder ähnliche Dinge beschädigt"), Zündeln (,gezündelt oder etwas in Brand gesteckt“). Die Skala Delinquenter Freundeskreis wurde aus den Angaben zum Ladendiebstahl, zur Körperverletzung, zu Rauchen/Alkohol und zum Zündeln gebildet. ${ }^{31}$ Die Angaben für das Delikt, bei dem die höchste Zahl eingetragen wurde, wurden als Indikator für die Anzahl delinquenter Freunde herangezogen.

\section{Selbstkontrolle}

00045

In den Schülerbefragungen des KFN wurden als drei zentrale Subdimensionen geringer Selbstkontrolle unbeständiges oder aufbrausendes Temperament, Risikosuche und Impulsivität gemessen (vgl. Baier, 2008; Wilmers et al., 2002; Mößle et al., 2007). Die Skalen für aufbrausendes Temperament und Risikosuche wurden, teilweise in gekürzter Form, für diesen Längsschnitt in der fünften und sechsten Klasse übernommen. Impulsivität wurde bereits von der vierten

31 Da Sachbeschädigung nur in der fünften Klasse abgefragt wurde, wurde aus Gründen der Vergleichbarkeit zwischen vierter und fünfter Klasse auf dieses Item zur Skalenbildung verzichtet. 
Klasse an zusammen mit Empathie mittels des IVE, dem Inventar zur Erfassung von Impulsivität, Risikoverhalten und Empathie bei 9-bis 14-jährigen Kindern (Stadler et al., 2004a), erfasst.

Aufbrausendes Temperament. Aus den drei vierfach abgestuften (,stimmt gar nicht“" (1), stimmt kaum“ (2), stimmt ziemlich“ (3), stimmt genau“ (4)) Items „Ich verliere ziemlich schnell die Beherrschung“, „Wenn ich mit jemandem wirklich Streit habe, kann ich nur schwer ruhig bleiben“ und „Wenn ich echt wütend bin, gehen mir die Anderen besser aus dem Weg“" wurde die Kurzskala aufbrausendes Temperament ${ }^{32}$ gebildet. Hohe Werte auf dieser Skala stehen für ein aufbrausendes Temperament, d. h. einer Schwierigkeit der Kinder, aggressive Impulse zu kontrollieren. Mit einem Cronbachs $\alpha_{5}$. Klasse $=.65$ bzw. Cronbachs $\alpha_{6 . \text { Klasse }}=.70$ ist bei insgesamt drei Items eine akzeptable bis befriedigende interne Konsistenz der Skala gegeben, die sich geringfügig bis deutlich unter dem Niveau der Ausgangsskala bewegt (Skalen- und Itemkennwerte siehe Tabelle 15). Bei im Durchschnitt 15-jährigen Jugendlichen lag die interne Konsistenz in der KFN-Schülerbefragung 2005 zum Vergleich bei Cronbachs $\alpha=.76$ (vgl. Baier et al., 2006).

Tabelle 15. Skalen- und Itemkennwerte der Skala aufbrausendes Temperament nach Messzeitpunkt.

\begin{tabular}{|c|c|c|c|c|}
\hline & \multicolumn{2}{|c|}{ 5. Klasse } & \multicolumn{2}{|c|}{ 6. Klasse } \\
\hline & $\begin{array}{c}M \\
(S D)\end{array}$ & $\overline{r_{i t}}$ & $\begin{array}{c}M \\
(S D)\end{array}$ & $\boldsymbol{r}_{i t}$ \\
\hline Ich verliere ziemlich schnell die Beherrschung. & $\begin{array}{l}1.78 \\
(.89)\end{array}$ & .46 & $\begin{array}{l}1.67 \\
(.82)\end{array}$ & .53 \\
\hline $\begin{array}{l}\text { Wenn ich mit jemandem wirklich Streit habe, kann ich nur } \\
\text { schwer ruhig bleiben. }\end{array}$ & $\begin{array}{c}2.34 \\
(1.04)\end{array}$ & .49 & $\begin{array}{l}2.24 \\
(.99)\end{array}$ & .54 \\
\hline $\begin{array}{l}\text { Wenn ich echt wütend bin, gehen mir die Anderen besser } \\
\text { aus dem Weg. }\end{array}$ & $\begin{array}{c}2.18 \\
(1.07)\end{array}$ & .43 & $\begin{array}{c}2.06 \\
(1.03)\end{array}$ & .48 \\
\hline$M(S D)$ & \multicolumn{2}{|c|}{$2.10(.77)$} & \multicolumn{2}{|c|}{$1.99(.75)$} \\
\hline Cronbachs $\alpha$ & \multicolumn{2}{|c|}{.65} & \multicolumn{2}{|c|}{.70} \\
\hline
\end{tabular}

Anmerkung. $r_{i t}=$ Trennschärfe (Part-Whole-Korrektur). Skalenwerte von 1 bis 4 (,stimmt gar nicht“, ,stimmt kaum“ ,stimmt ziemlich“, ,stimmt genau“).

32 Mittelwert der drei Items. Die beiden Items „Bei einer ernsten Meinungsverschiedenheit ist es normalerweise schwer für mich, ruhig darüber zu reden, ohne mich aufzuregen“ und „Wenn ich mich über andere ärgere habe ich oft mehr Lust sie zu verletzen, als mit ihnen darüber zu reden“ wurden nicht in die Kurzskala des Fragebogens aufgenommen (vgl. Baier et al., 2006). 
Risikosuche. In die Skala Risikosuche ${ }^{33}$ (Skalen- und Itemkennwerte siehe Tabelle 16) gingen folgende vier, wiederum vierfach abgestuften (,stimmt gar nicht“ (1), stimmt kaum“ (2), stimmt ziemlich“ (3), stimmt genau“ (4)) Items ein: „Ich teste gerne meine Grenzen, indem ich etwas Gefährliches mache“, „Ich gehe gern ein Risiko ein, einfach weil es Spaß macht“, „Aufregung und Abenteuer sind mir wichtiger als Sicherheit" und „Manchmal finde ich es aufregend, Dinge zu tun, die mich in Gefahr bringen können“ (vgl. Baier et al., 2006). Hohe Werte stehen dabei für ein risikofreudiges Verhalten, also die Neigung, Spannung und Abenteuer durch riskante und aufregende Tätigkeiten zu erleben. Mit einem Cronbachs $\alpha_{5 \text {. Klasse }}=.84$ bzw. Cronbachs $\alpha_{6 . \text { Klasse }}=.88$ ist bei insgesamt vier Items eine gute interne Konsistenz der Skala gegeben, die mit der Ausgangsskala der KFN-Schülerbefragung 2005 (Cronbachs $\alpha=.87$ ) vergleichbar ist (vgl. Baier et al., 2006).

Tabelle 16. Skalen- und Itemkennwerte der Skala Risikosuche nach Messzeitpunkt.

\begin{tabular}{|c|c|c|c|c|}
\hline & \multicolumn{2}{|c|}{ 5. Klasse } & \multicolumn{2}{|c|}{ 6. Klasse } \\
\hline & $\begin{array}{c}M \\
(S D)\end{array}$ & $\boldsymbol{r}_{i t}$ & $\begin{array}{c}M \\
(S D)\end{array}$ & $\boldsymbol{r}_{i t}$ \\
\hline $\begin{array}{l}\text { Ich teste gerne meine Grenzen, indem ich etwas Gefährli- } \\
\text { ches mache. }\end{array}$ & $\begin{array}{l}1.59 \\
(.87)\end{array}$ & .68 & $\begin{array}{l}1.67 \\
(.87)\end{array}$ & .74 \\
\hline Ich gehe gern ein Risiko ein, einfach weil es Spaß macht. & $\begin{array}{l}1.65 \\
(.89)\end{array}$ & .71 & $\begin{array}{l}1.78 \\
(.92)\end{array}$ & .77 \\
\hline Aufregung und Abenteuer sind mir wichtiger als Sicherheit. & $\begin{array}{l}1.60 \\
(.87)\end{array}$ & .61 & $\begin{array}{l}1.79 \\
(.90)\end{array}$ & .68 \\
\hline $\begin{array}{l}\text { Manchmal finde ich es aufregend, Dinge zu tun, die mich } \\
\text { in Gefahr bringen können. }\end{array}$ & $\begin{array}{l}1.43 \\
(.78)\end{array}$ & .70 & $\begin{array}{l}1.69 \\
(.90)\end{array}$ & .74 \\
\hline$M(S D)$ & \multicolumn{2}{|c|}{$1.57(.70)$} & \multicolumn{2}{|c|}{$1.74(.77)$} \\
\hline Cronbachs $\alpha$ & \multicolumn{2}{|c|}{.84} & \multicolumn{2}{|c|}{.88} \\
\hline
\end{tabular}

Anmerkung. $r_{i t}=$ Trennschärfe (Part-Whole-Korrektur). Skalenwerte von 1 bis 4 (,stimmt gar nicht“, ,stimmt kaum“ „stimmt ziemlich“, ,stimmt genau“).

\section{Schuleinstellung}

Schuleinstellung wurde im Schülerfragebogen der fünften und sechsten Klasse abgefragt. Zur Bildung der „Kurzskala Schuleinstellung“ wurden die 3 trennschärfsten der insgesamt 14 Items der Skala Schuleinstellung des Fragebogens zur Erfassung emotionaler und sozialer Schulerfahrungen von Grundschulkindern dritter und vierter Klassen (FEESS 3-4, SALGA; Rauer \& Schuck, 2003)

33 Mittelwert der vier Items. 
ausgewählt. Folgende drei Items gingen in die Skala Schuleinstellung ${ }^{34}$ ein: „Morgens freue ich mich auf die Schule“, „Schule macht Spaß“, „Ich gehe gern zur Schule“. Hohe Werte auf dieser Skala stehen für Freude und Spaß an der Schule. Mit einem Cronbachs $\alpha_{5 \text {. Klasse }}=.87$ bzw. Cronbachs $\alpha_{6 . \text { Klasse }}=.90$ ist bei insgesamt drei Items eine gute bis sehr gute interne Konsistenz der Skala gegeben, die leicht unter dem der Eichstichprobe des FEESS mit 14 Items liegt (Cronbachs $\alpha_{3 . \text { Klasse }}=.94$ bzw. Cronbachs $\alpha_{4 \text {. Klasse }}=.95$; Skalen- und Itemkennwerte siehe Tabelle 17).

Tabelle 17. Skalen- und Itemkennwerte der Skala Schuleinstellung nach Messzeitpunkt.

\begin{tabular}{|c|c|c|c|c|c|}
\hline & \multicolumn{2}{|c|}{ 5. Klasse } & \multicolumn{2}{|c|}{ 6. Klasse } \\
\hline & & $\begin{array}{c}M \\
(S D)\end{array}$ & $\overline{r_{i t}}$ & $\begin{array}{c}M \\
(S D)\end{array}$ & $\overline{r_{i t}}$ \\
\hline \multicolumn{2}{|l|}{ Morgens freue ich mich auf die Schule. } & $\begin{array}{l}2.24 \\
(.97)\end{array}$ & .72 & $\begin{array}{l}2.14 \\
(.91)\end{array}$ & .78 \\
\hline \multicolumn{2}{|l|}{ Schule macht Spaß. } & $\begin{array}{l}2.58 \\
(.97)\end{array}$ & .76 & $\begin{array}{l}2.36 \\
(.94)\end{array}$ & .78 \\
\hline \multirow[t]{3}{*}{ Ich gehe gern zur Schule. } & & $\begin{array}{l}2.48 \\
(1.0)\end{array}$ & .81 & $\begin{array}{l}2.35 \\
(.98)\end{array}$ & .84 \\
\hline & $M(S D)$ & \multicolumn{2}{|c|}{$2.44(.88)$} & \multicolumn{2}{|c|}{$2.99(.86)$} \\
\hline & Cronbachs $\alpha$ & \multicolumn{2}{|c|}{.87} & \multicolumn{2}{|c|}{.90} \\
\hline
\end{tabular}

Anmerkung. $r_{i t}=$ Trennschärfe (Part-Whole-Korrektur). Skalenwerte von 1 bis 4 (,stimmt nicht“, „stimmt kaum“",stimmt ziemlich“, „stimmt genau“).

\section{Depressive Verstimmung}

02345

Das allgemeine Befinden der Schülerinnen und Schüler wurde vom zweiten Messzeitpunkt am Ende der dritten Klasse an mit Hilfe des Kiddo-KINDL ${ }^{\mathrm{R}}$ (Ravens-Sieberer \& Bullinger, 2000) erhoben. Um das Vorhandensein einer Depression als einer häufig berichteten Komorbidität bei computerspielabhängigen Erwachsenen (vgl. te Wildt, Putzig, Zedler \& Ohlmeier, 2007) auch für unsere Kinderstichprobe näherungsweise abschätzen zu können, wurde aus folgenden fünfstufigen Items eine Skala depressive Verstimmung ${ }^{35}$ gebildet (die allerdings nicht mit einer klinischen Diagnose gleichzusetzen ist): „In der letzten Woche habe ich viel gelacht“, „In der letzten Woche war mir langweilig“ (umgepolt), „In der letzten Woche hatte ich viel Kraft und Ausdauer“, „In der letzten Woche war ich tagsüber müde und schlapp“ (umgepolt) sowie „In der letzten Woche habe ich

34 Mittelwert der drei Items.

35 Summenscore der fünfstufigen Antworten. 
mich allein gefühlt"“ (umgepolt) (vgl. alle Kiddo-KINDLR; Ravens-Sieberer \& Bullinger, 2000). Trotz schlechter bis akzeptabler Werte der internen Konsistenz (Cronbachs $\alpha_{4 . \text { Klasse }}=.59$, Cronbachs $\alpha_{4 . \text { Klasse }}=.68$, Cronbachs $\alpha_{5 . \text { Klasse }}=.63$, Cronbachs $\alpha_{6 . \text { Klasse }}=.65$; Skalen- und Itemkennwerte siehe Tabelle 18), wurde die Skala ${ }^{36}$ in die Auswertungen mit einbezogen, um zumindest einen Schätzer einer depressiven Verstimmung für den Berliner Längsschnitt Medien zu erhalten.

Tabelle 18. Skalen- und Itemkennwerte der Skala Depressive Verstimmung nach Messzeitpunkt.

\begin{tabular}{|c|c|c|c|c|c|c|c|c|}
\hline & \multicolumn{2}{|c|}{ 3. Klasse } & \multicolumn{2}{|c|}{ 4. Klasse } & \multicolumn{2}{|c|}{ 5. Klasse } & \multicolumn{2}{|c|}{ 6. Klasse } \\
\hline & $\begin{array}{c}M \\
(S D)\end{array}$ & $\overline{r_{i t}}$ & $\begin{array}{c}M \\
(S D)\end{array}$ & $\overline{r_{i t}}$ & $\begin{array}{c}M \\
(S D)\end{array}$ & $\overline{r_{i t}}$ & $\begin{array}{c}M \\
(S D)\end{array}$ & $\overline{r_{i t}}$ \\
\hline $\begin{array}{l}\text { In der letzten Woche } \\
\text { habe ich viel gelacht } \\
\text { und viel Spaß gehabt. }\end{array}$ & $\begin{array}{l}1.87 \\
(.97)\end{array}$ & .37 & $\begin{array}{l}1.82 \\
(.89)\end{array}$ & .39 & $\begin{array}{l}1.86 \\
(.84)\end{array}$ & .38 & $\begin{array}{l}1.85 \\
(.86)\end{array}$ & .40 \\
\hline $\begin{array}{l}\text { In der letzten Woche } \\
\text { war mir langweilig. }\end{array}$ & $\begin{array}{c}2.11 \\
(1.12)\end{array}$ & .37 & $\begin{array}{l}2.09 \\
(.99)\end{array}$ & .42 & $\begin{array}{l}2.20 \\
(.99)\end{array}$ & .40 & $\begin{array}{c}2.44 \\
(1.05)\end{array}$ & .42 \\
\hline $\begin{array}{l}\text { In der letzten Woche } \\
\text { hatte ich viel Kraft und } \\
\text { Ausdauer. }\end{array}$ & $\begin{array}{l}2.24 \\
(1.2)\end{array}$ & .31 & $\begin{array}{c}2.26 \\
(1.14)\end{array}$ & .47 & $\begin{array}{l}2.30 \\
(1.15)\end{array}$ & .38 & $\begin{array}{c}2.42 \\
(1.12)\end{array}$ & .34 \\
\hline $\begin{array}{l}\text { In der letzten Woche } \\
\text { war ich tagsüber müde } \\
\text { und schlapp. }\end{array}$ & $\begin{array}{l}1.88 \\
(1.11)\end{array}$ & .31 & $\begin{array}{c}1.92 \\
(1.16)\end{array}$ & .47 & $\begin{array}{c}1.87 \\
(1.06)\end{array}$ & .45 & $\begin{array}{c}2.03 \\
(1.10)\end{array}$ & .41 \\
\hline $\begin{array}{l}\text { In der letzten Woche } \\
\text { habe ich mich allein ge- } \\
\text { fühlt. }\end{array}$ & $\begin{array}{l}1.54 \\
(.99)\end{array}$ & .37 & $\begin{array}{l}1.44 \\
(.86)\end{array}$ & .41 & $\begin{array}{l}1.41 \\
(.84)\end{array}$ & .35 & $\begin{array}{l}1.50 \\
(.90)\end{array}$ & .45 \\
\hline$M(S D)$ & \multicolumn{2}{|c|}{$9.62(3.32)$} & \multicolumn{2}{|c|}{$9.52(3.35)$} & \multicolumn{2}{|c|}{$9.62(3.13)$} & \multicolumn{2}{|c|}{$10.23(3.26)$} \\
\hline Cronbachs $\alpha$ & \multicolumn{2}{|c|}{.59} & \multicolumn{2}{|c|}{.67} & \multicolumn{2}{|c|}{.63} & \multicolumn{2}{|c|}{.65} \\
\hline
\end{tabular}

Anmerkung. $r_{i t}=$ Trennschärfe (Part-Whole-Korrektur). Skalenwerte von 1 bis 5 (,immer“, „oft“ „manchmal“", „selten“, „nie“).

\section{Computerspielabhängigkeit}

00045

Nach der Hinzunahme des Schwerpunktes Kindergewalt in der dritten Welle (4. Klasse) des Längsschnittes wurde im Mai 2008 (5. Klasse) das Thema Computerspielabhängigkeit zum ersten Mal in die Befragung mit aufgenommen. Um das Risiko einer Computerspielabhängigkeit empirisch für die Berliner Stichprobe zu überprüfen, wurde zunächst die Skala Computerspielabhängigkeit der Schülerbefragung 2005 - KFN-CSAS-I (Grundlage bei der Entwicklung war die

36 Die Eindimensionalität der Skala konnte zudem faktorenanalytisch für alle Messzeitpunkte bestätigt werden. 
ISS-20; Hahn \& Jerusalem, 2001c; Rehbein \& Borchers, 2009; Mößle et al., 2007) eingesetzt. Vier zentrale Merkmale einer Abhängigkeit nach ICD-10 (Einengung des Denkens und Verhaltens, Kontrollverlust, Entzugserscheinungen und negative Konsequenzen) werden in dieser Skala mittels vierstufiger Items (,Stimmt nicht“, „Stimmt kaum“, „Stimmt eher“, „Stimmt genau“) im Selbstbericht erhoben. Zwei Items dieser Skala erfassen dabei Entzugserscheinungen: „Wenn ich nicht spielen kann, bin ich gereizt und unzufrieden“, „Wenn ich längere Zeit nicht spiele, werde ich unruhig und nervös“; vier Items Negative Konsequenzen: „Meine Leistungen in der Schule leiden unter meinen Spielgewohnheiten“ (Leistung), „Ich bin so häufig und intensiv mit Computerspielen beschäftigt, dass ich manchmal Probleme in der Schule bekomme“ (Leistung), „Mir wichtige Menschen beschweren sich, dass ich zu viel Zeit mit Spielen verbringe“ (sozial), „Weil ich so viel spiele, unternehme ich weniger mit Anderen“ (sozial); zwei Items Einengung des Denkens und Verhaltens: „Ich beschäftige mich auch während der Zeit, in der ich nicht Computerspiele spiele, gedanklich sehr viel mit Spielen“, „Meine Gedanken kreisen ständig um das Computer- und Videospielen, auch wenn ich gar nicht spiele“; sowie drei Items Kontrollverlust: „Beim Computerspielen ertappe ich mich häufig dabei, dass ich sage: ,Nur noch ein paar Minuten’ und dann kann ich doch nicht aufhören“, „Ich habe schon häufiger vergeblich versucht, meine Spielzeit zu reduzieren“, „Ich verbringe oft mehr Zeit mit Computer- und Videospielen, als ich mir vorgenommen habe“.

Tabelle 19. Skalen- und Itemkennwerte der Computerabhängigkeitsskalen KFNCSAS-I und KFN-CSAS-II. ${ }^{37}$

\begin{tabular}{c|ccc}
\hline & $\begin{array}{c}\text { 5. Klasse } \\
\text { KFN- } \\
\text { CSAS-I }\end{array}$ & $\begin{array}{c}\text { 6. Klasse } \\
\text { KFN- } \\
\text { CSAS-II }\end{array}$ \\
\hline $\begin{array}{c}M \\
\text { (SD) }\end{array}$ & $\begin{array}{r}M \\
\text { (SD) }\end{array}$ \\
\hline
\end{tabular}

\section{Einengung des Denkens und Verhaltens}

Ich beschäftige mich auch während der Zeit, in der ich nicht Computerspiele spiel, gedanklich sehr viel mit Spielen.

1.75

Meine Gedanken kreisen ständig ums Computer- und Videospielen, auch wenn ich gar nicht spiele.

1.28

$\mathrm{Zu}$ bestimmten Zeiten oder in bestimmten Situationen spiele ich eigentlich immer: Das ist fast zu einer Routine für mich geworden.

$\begin{array}{lll}.45 & 1.64 & \\ & (.86) & .58 \\ & & \\ & 1.32 & \\ & (.70) & .77 \\ & & \\ & 1.36 & \\ & (.69) & .67 \\ & & \end{array}$

37 Bei Vorliegen nur eines Missingwertes wurde dieser durch den empirischen Itemmittelwert ersetzt. Bei mehr als einem Missingwert wurde jeweils die gesamte Skala als missing gewertet. 


\begin{tabular}{cccc}
$\begin{array}{c}\text { 5. Klasse } \\
\text { KFN- }\end{array}$ & \multicolumn{2}{c}{ 6. Klasse } \\
KFN- \\
CSAS-I & \multicolumn{2}{c|}{ CSAS-II } \\
\hline$M$ & $r_{i t}$ & $\begin{array}{c}M \\
\text { (SD) }\end{array}$ & $r_{i t}$ \\
(SD) & & (SD) \\
& & 1.29 & .70 \\
& & $(.65)$ & \\
\hline
\end{tabular}

Es kommt vor, dass ich eigentlich etwas ganz anderes tue und dann ohne zu überlegen ein Computerspiel starte.

\section{Negative Konsequenzen}

Meine Leistungen in der Schule leiden unter meinen Spielgewohnheiten.

$\begin{array}{llll}1.24 & .53 & 1.32 & .63 \\ (.60) & & (.69) & \\ 1.21 & & 1.24 & \\ (.56) & .58 & (.60) & .72 \\ & & & \\ 1.32 & .53 & 1.34 & .70 \\ (.71) & & (.66) & \\ 1.27 & .49 & 1.37 & .65 \\ (.60) & .72) & (.65\end{array}$

\section{Kontrollverlust}

Beim Computerspielen ertappe ich mich häufig dabei, dass ich sage: ,Nur noch ein paar Minuten' und dann kann ich doch nicht aufhören.

Ich verbringe oft mehr Zeit mit Computer- und Videospielen, als ich mir vorgenommen habe.

Ich habe das Gefühl, meine Spielzeit nicht kontrollieren zu können.

\section{Entzugserscheinungen}

Wenn ich nicht spielen kann, bin ich gereizt und unzufrieden.

Wenn ich längere Zeit nicht spiele, werde ich unruhig und nervös.

\section{Toleranzentwicklung}

Ich habe das Gefühl, dass Video- und Computerspiele für mich immer wichtiger werden.

Ich muss immer länger spielen, um zufrieden zu sein.

Anmerkung. $r_{i t}=$ Trennschärfe (Part-Whole-Korrektur). Skalenwerte von 1 bis 4 (,stimmt nicht“, „stimmt kaum“",stimmt eher“, „stimmt genau“).

Bei elf Items auf einer vierstufigen Skala (Cronbachs $\alpha_{5 \text {. Klasse }}=.85$, Skalen- und Itemkennwerte siehe Tabelle 19) resultieren Gesamtsummenwerte zwischen 11 und 44 mit der folgenden Gruppeneinteilung: Rohwertsumme $11-27=$ unauf- 
fällig, Rohwertsumme 28 - 32 = gefährdet im Sinne einer Abhängigkeitsproblematik, Rohwertsumme $33-44$ = abhängig (vgl. Mößle et al., 2007; Rehbein \& Borchers, 2009).

Ab Mai 2009 (6. Klasse) wurde im Berliner Längsschnitt Medien die für die KFN-Schülerbefragung 2007/2008 von uns weiterentwickelte Computerspielabhängigkeitsskala KFN-CSAS-II (vgl. Rehbein et al., 2009a; Rehbein, Kleimann \& Mößle, 2009b, 2010) eingesetzt. ${ }^{38}$ Im Vergleich zur KFN-CSAS-I wird in dieser zusätzlich das Merkmal der Toleranzentwicklung mit den Items „Ich habe das Gefühl, dass Video- und Computerspiele für mich immer wichtiger werden“" sowie „Ich muss immer länger spielen, um zufrieden zu sein“ mit erhoben. Die Dimension Einengung den Denkens und Verhaltens wurde des Weiteren um zwei weitere Items ergänzt: „Zu bestimmten Zeiten oder in bestimmten Situationen spiele ich eigentlich immer: Das ist fast zu einer Routine für mich geworden“, „Es kommt vor, dass ich eigentlich etwas ganz anderes tue und dann ohne zu überlegen ein Computerspiel starte“. Beim Merkmal Kontrollverlust wurde zudem das Item „Beim Computerspielen ertappe ich mich häufig dabei, dass ich sage: ,Nur noch ein paar Minuten' und dann kann ich doch nicht aufhören“ gestrichen. Erhoben werden in der KFN-CSAS-II somit die Dimensionen Einengung des Denkens und Verhaltens (4 Items), Negative Konsequenzen (4 Items), Kontrollverlust (2 Items), Entzugserscheinungen (2 Items) und Toleranzentwicklung (2 Items). Bei nunmehr 14 Items auf einer vierstufigen Skala (Cronbachs $\alpha_{6 . \text { Klas- }}$ se $=.94$, Skalen- und Itemkennwerte siehe Tabelle 19) resultieren Gesamtsummenwerte zwischen 14 und 56 mit der folgenden Gruppeneinteilung der diagnostischen Cut-off-Werte: Rohwertsumme 14 - 34 = unauffällig, Rohwertsumme 35 - 41 = gefährdet im Sinne einer Abhängigkeitsproblematik, Rohwertsumme 42 56 = abhängig (vgl. Rehbein et al., 2009a, 2009b, 2010).

\subsubsection{Standardisierte Testverfahren}

\section{CFT-20 - WS - ZF}

\section{0}

Zur Bestimmung der allgemeinen Intelligenz der Schülerinnen und Schüler kam von der dritten bis zur fünften Klasse der Grundintelligenztest Skala 2 (CFT 20), die deutsche Adaptation des Culture Fair Intelligence Test - Scale 2 von Cattell (1960) als ein „ökonomisches psychologisches Verfahren zur validen Diagnose der grundlegende geistigen Leistungsfähigkeit" (Weiß, 1998a, S. 8) zum Einsatz. Das Verfahren wurde ausgewählt, da dieses so konstruiert wurde, dass es weitgehend ,frei ist von - mehr oder weniger zufälligen - Einflüssen des soziokulturel- 
len, erziehungsspezifischen oder rassischen Hintergrundes“" (Weiß, 1998a, S. 8). Aus Gründen der Befragungsökonomie wurde nur der erste von zwei äquivalenten Testteilen durchgeführt. Zur Bestimmung der verbalen sowie numerischen Fähigkeiten und Fertigkeiten wurden zudem die Erweiterungen Zahlenfolgetest (ZF) und Wortschatztest (WS) eingesetzt (beide Weiß, 1998b). Zur Umrechnung in IQ-Werte wurden die jeweiligen Altersnormwerte zu Grunde gelegt (Kennwerte siehe Tabelle 20).

Tabelle 20. Kennwerte des CFT 20, des WS bzw. des ZF .

\begin{tabular}{lcccccccccccc}
\hline & \multicolumn{3}{c}{ 3. Klasse HJ } & \multicolumn{3}{c}{ 3. Klasse } & \multicolumn{3}{c}{ 4. Klasse } & \multicolumn{3}{c}{ 5. Klasse } \\
\cline { 2 - 14 } & $\mathbf{N}$ & $M$ & SD & $\mathbf{N}$ & $M$ & SD & $\mathbf{N}$ & $M$ & SD & $\mathbf{N}$ & $M$ & SD \\
\hline CFT 20 & 906 & 105 & 17 & 812 & 109 & 17 & 790 & 111 & 16 & 794 & 110 & 16 \\
WS & 911 & 99 & 14 & 810 & 102 & 14 & 769 & 103 & 14 & 797 & 101 & 13 \\
\hline ZF & 879 & 102 & 11 & 801 & 103 & 13 & 769 & 104 & 15 & 796 & 101 & 15 \\
\hline
\end{tabular}

Tabelle 20 kann entnommen werden, dass die Berliner Grundschülerinnen und Grundschüler bereits in der dritten Klasse IQ-Werte zeigen, welche leicht oberhalb des Mittelwerts liegen. Abbildung 17 veranschaulicht, dass dies vor allem auf eine positive Abweichung im leicht überdurchschnittlichen Intelligenzbereich zurückzuführen ist. ${ }^{39}$ Die leichte Mittelwertszunahme von der dritten bis zur fünften Klasse ist auf Messwiederholungseffekte zurückzuführen und liegt von der dritten bis zur fünften Klasse auf einem zu erwartendem Niveau (Zugewinn bei 14-tätiger Retestung: 5,4 Rohpunkte; Weiß, 1998a, S. 44). Die verbalen sowie numerischen Fähigkeiten und Fertigkeiten bleiben von der dritten bis zur fünften Klasse bei leichten Schwankungen relativ stabil und entsprechen dem Mittelwert von 100 IQ-Punkten.

39 Die leicht überdurchschnittlichen IQ-Werte sollten aber nicht im Sinne des Flynn-Effektes (vgl. Flynn, 1984, 1987, 1999), eines ,Trend des Intelligenzanstiegs, gemessen durch (vorwiegend sprachfreie) Tests" (Oerter, 2008, S. 255), der in industrialisierten Staaten beobachtet werden konnte (vgl. Bullock \& Ziegler, 1997) interpretiert werden. In den Daten des Berliner Längsschnitts Medien wäre dieser Effekt mit 5 IQ-Punkten zudem vergleichsweise gering ausgeprägt. 


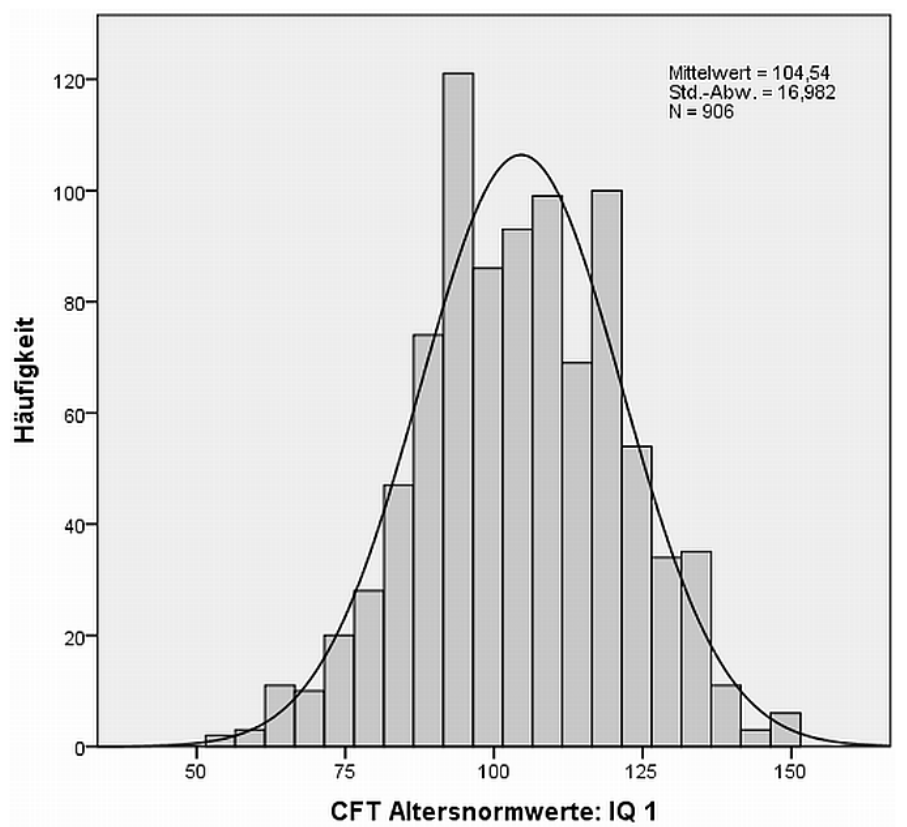

Abbildung 17. Histogramm IQ (CFT 20) dritte Klasse.

FEESS 3-4

192345

In der dritten und vierten Klasse wurde das Selbstkonzept eigener Schulfähigkeiten sowie die erlebte soziale Integration mit dem Fragebogen zur Erfassung emotionaler und sozialer Schulerfahrungen von Grundschulkindern dritter und vierter Klassen (FEESS 3-4, SIKS; Rauer \& Schuck, 2003) erfasst. Derr FEESS 3-4 ,ist ein Verfahren zur Erfassung der psychologisch bedeutsamen und pädagogisch relevanten Sichtweisen, Einschätzungen, Bewertungen und Einstellungen von Grundschulkindern. Erfasst werden die Kompetenzerwartungen der Schülerinnen und Schüler, die erlebte soziale, emotionale und leistungsmotivationale Integration, die wahrgenommenen sozialen Beziehungen in der Klasse, das wahrgenommene Schul- und Lernklima und die Art und Weise, wie die Schülerinnen und Schüler die Lehrer und Lehrerinnen und die Schule insgesamt wahrnehmen. Es handelt sich dabei um in schulischen Kontexten erworbene, überdauernde, generalisierte und situationsunabhängige Überzeugungen." (Rauer \& Schuck, 2003, S. 9) Integration misst dabei das „Ausmaß, in dem ein Kind sich durch die Mitschüler und Mitschülerinnen angenommen fühlt und sich selbst als vollwertiges Gruppenmitglied betrachtet" (Rauer \& Schuck, 2003, S. 9). Selbstkonzept der Schulfähigkeit steht dabei für das „Ausmaß, in dem ein Kind sich den schuli- 
schen Aufgaben gewachsen fühlt und seine schulischen Fähigkeiten positiv bewertet" (Rauer \& Schuck, 2003, S. 9).

$\mathrm{Da}$ der FEESS 3-4 in seiner ursprünglichen Konzeption lediglich für das Grundschulalter bis zur vierten Klasse konzipiert ist und somit keine Normwerte für ältere Jahrgangsstufen vorliegen, kam in der fünften und sechsten Klasse, um dennoch im Längsschnitt vergleichbare Daten zu erlangen, jeweils eine „Kurzskala Selbstkonzept eigener Fähigkeiten“ sowie „soziale Integration“ zum Einsatz. Zur Bildung der Kurzskalen Selbstkonzept eigener Schulfähigkeiten und Soziale Integration wurden die 4 bzw. 3 trennschärfsten der insgesamt 15 bzw. 11 Items der Eichstichprobe des FEESS (SIKS; Rauer \& Schuck, 2003) ausgewählt. Die Kurzskala Selbstkonzept eigener Schulfähigkeiten bildet sich aus dem Summenscore der vierstufigen (,stimmt nicht“ (1), „stimmt kaum“ (2), „stimmt ziemlich“ (3), „stimmt genau“ (4)) Items „Ich lerne sehr langsam (umgepolt)“, „Ich mache in der Schule das meiste richtig“, „Ich kann ganz gut lernen“, „Ich bin gut in der Schule". Hohe Werte auf dieser Skala stehen für eine hohe Einschätzung der schulischen Fähigkeiten. Mit einem Cronbachs $\alpha_{5 \text {. Klasse }}=.84 \mathrm{bzw}$. Cronbachs $\alpha_{6 . \text { Klasse }}=.87$ ist bei insgesamt vier Items eine gute interne Konsistenz der Skala gegeben, die sich auf vergleichbarem Niveau wie bei der Ausgangsskala bewegt (die interne Konsistenz lag in der Eichstichprobe zum Vergleich bei Cronbachs $\alpha_{3 . \text { Klasse }}=.79$ bzw. Cronbachs $\alpha_{4 \text {. Klasse }}=.84$; Skalen- und Itemkennwerte siehe Tabelle 21).

Tabelle 21. Skalen- und Itemkennwerte der Kurzskala Selbstkonzept eigener Schulfähigkeiten nach Messzeitpunkt.

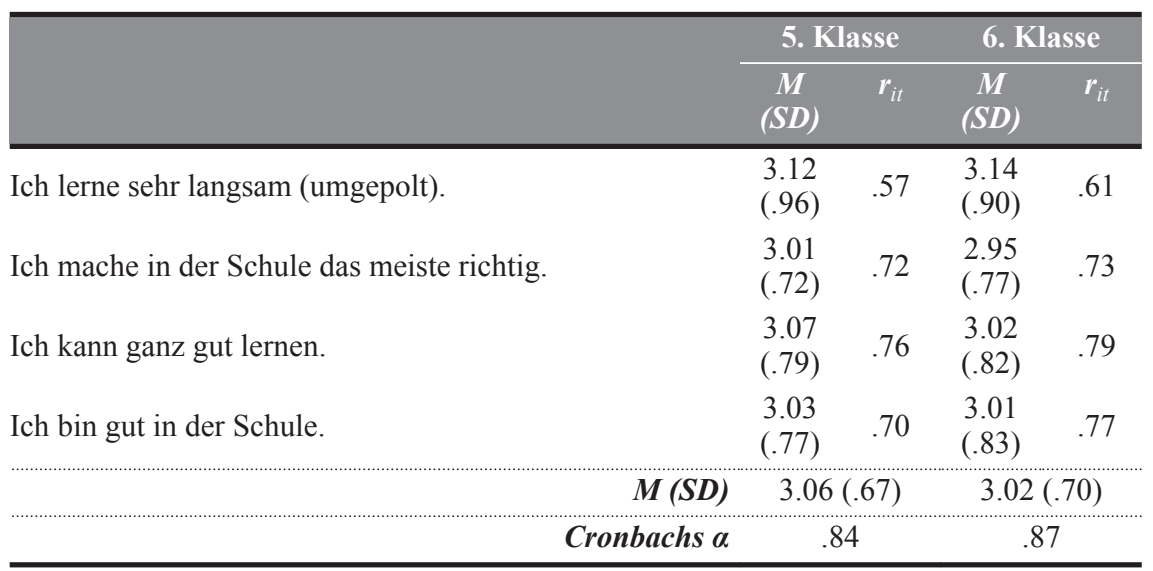

Anmerkung. $r_{i t}=$ Trennschärfe (Part-Whole-Korrektur). Skalenwerte von 1 bis 4 (,stimmt nicht“, „stimmt kaum“",stimmt ziemlich“, ,stimmt genau“). 
Tabelle 22. Skalen- und Itemkennwerte der Kurzskala Soziale Integration nach Messzeitpunkt.

\begin{tabular}{|c|c|c|c|c|}
\hline & \multicolumn{2}{|c|}{ 5. Klasse } & \multicolumn{2}{|c|}{ 6. Klasse } \\
\hline & $\begin{array}{c}M \\
(S D)\end{array}$ & $r_{i t}$ & $\begin{array}{c}M \\
(S D)\end{array}$ & $\boldsymbol{r}_{i t}$ \\
\hline Meine Mitschüler sind nett zu mir. & $\begin{array}{l}3.22 \\
(.78)\end{array}$ & .62 & $\begin{array}{l}3.22 \\
(.79)\end{array}$ & .69 \\
\hline $\begin{array}{l}\text { Ich komme mit anderen Kindern in meiner Klasse gut } \\
\text { aus. }\end{array}$ & $\begin{array}{l}3.21 \\
(.79)\end{array}$ & .55 & $\begin{array}{l}3.27 \\
(.78)\end{array}$ & .67 \\
\hline Ich habe wenig Freunde in meiner Klasse. & $\begin{array}{l}3.39 \\
(.94)\end{array}$ & .48 & $\begin{array}{l}3.47 \\
(.83)\end{array}$ & .54 \\
\hline$M(S D)$ & \multicolumn{2}{|c|}{$3.26(.68)$} & \multicolumn{2}{|c|}{$3.32(.67)$} \\
\hline Cronbachs $\alpha$ & \multicolumn{2}{|c|}{.72} & \multicolumn{2}{|c|}{.79} \\
\hline
\end{tabular}

Anmerkung. $r_{i t}=$ Trennschärfe (Part-Whole-Korrektur). Skalenwerte von 1 bis 4 (,stimmt nicht“, „stimmt kaum“",stimmt ziemlich“, „stimmt genau“).

Die Kurzskala Soziale Integration setzt sich folgendermaßen zusammen: Summenscore der vierstufigen (,stimmt nicht“ (1), „stimmt kaum“ (2), „stimmt ziemlich“ (3), „stimmt genau“ (4)) Items „Meine Mitschüler sind nett zu mir“, „Ich komme mit anderen Kindern in meiner Klasse gut aus“, „Ich habe wenig Freunde in meiner Klasse“ (umgepolt). Hohe Werte auf dieser Skala stehen für eine gute Integration in die Klassengemeinschaft. Mit einem Cronbachs $\alpha_{5 \text {. Klas- }}$ $s_{s e}=.72$ bzw. Cronbachs $\alpha_{6 . \text { Klasse }}=.79$ ist bei drei Items eine befriedigende interne Konsistenz der Kurzskala soziale Integration gegeben, die allerdings etwas geringer ausfällt als in der Ausgangsskala (die interne Konsistenz lag in der Eichstichprobe zum Vergleich bei Cronbachs $\alpha_{3 \text {. Klasse }}=.84$ bzw. Cronbachs $\alpha_{4 \text {. Klas- }}$ $s e=.85$; Skalen- und Itemkennwerte siehe Tabelle 22).

\section{IVE}

Die Persönlichkeitseigenschaften Impulsivität und Empathie wurden von der vierten bis zur sechsten Klasse mittels des IVE, dem Inventar zur Erfassung von Impulsivität, Risikoverhalten und Empathie bei 9-bis 14-jährigen Kindern (Stadler et al., 2004a), einer adaptierten Form des Impulsivitätsfragebogens $I_{6}$ von Eysenck und Eysenck (Eysenck \& Eysenck, 1980) erfasst. Wiederum aus Befragungsökonomischen Gründen kam für beide Konstrukte eine Kurzskala zum Einsatz. Aus den folgenden vier der insgesamt 16 Impulsivitätsitems wurde eine Kurzskala Impulsivität (Summenscore der dichotomen Antworten) gebildet: „Ich tue und sage oft etwas, ohne darüber nachgedacht zu haben“, „Ich gerate oft in Schwierigkeiten, weil ich etwas tue, ohne zu überlegen“, „Ich gerate oft in unangenehme Situationen, weil ich vorher nicht genügend nachgedacht habe“, „Ich 
gerate oft in Schwierigkeiten, weil ich mich nicht genügend beherrschen kann“. Hohe Werte auf dieser Skala stehen für eine mangelnde Voraussicht eigener Handlungskonsequenzen, eine Orientierung an sofort verfügbaren positiven Konsequenzen eigener Handlungen und kaum Ausrichtung an zukünftigen Zielen, sowie einen schnellen und ungenauen Arbeitsstil (vgl. Stadler et al., 2004a). Mit einem Cronbachs $\alpha_{4 \text {. Klasse }}=.72$, Cronbachs $\alpha_{5 \text {. Klasse }}=.74$, bzw. Cronbachs $\alpha_{6 . \text { Klasse }}=.71$ ist bei insgesamt vier Items eine befriedigende interne Konsistenz der Skala gegeben, die sich etwas bis deutlich unter dem Niveau der Ausgangsskala bewegt (die interne Konsistenz lag in der Eichstichprobe zum Vergleich bei Cronbachs $\alpha_{10}$ Jahre, Jungen $=.77$, Cronbachs $\alpha_{10}$ Jahre, Mädchen $=.76$, Cronbachs $\alpha_{11 \text { Jahre, Jungen }}=.80$, Cronbachs $\alpha_{11 \text { Jahre, Mädchen }}=.78$, Cronbachs $\alpha_{12 \text { Jahre, Jungen }}=$. 86, Cronbachs $\alpha_{12}$ Jahre, Mädchen $=.78$; Skalen- und Itemkennwerte siehe Tabelle 23).

Tabelle 23. Skalen- und Itemkennwerte der Skala Impulsivität nach Messzeitpunkt.

\begin{tabular}{|c|c|c|c|c|c|c|}
\hline & \multicolumn{2}{|c|}{ 4. Klasse } & \multicolumn{2}{|c|}{ 5. Klasse } & \multicolumn{2}{|c|}{ 6. Klasse } \\
\hline & $\begin{array}{c}M \\
(S D)\end{array}$ & $\overline{r_{i t}}$ & $\begin{array}{c}M \\
(S D)\end{array}$ & $\boldsymbol{r}_{i t}$ & $\begin{array}{c}M \\
(S D)\end{array}$ & $\boldsymbol{r}_{i t}$ \\
\hline $\begin{array}{l}\text { Ich tue und sage oft etwas, ohne darüber } \\
\text { nachgedacht zu haben. }\end{array}$ & $\begin{array}{l}.34 \\
(.47)\end{array}$ & .47 & $\begin{array}{c}.44 \\
(.50)\end{array}$ & .46 & $\begin{array}{l}.45 \\
(.50)\end{array}$ & .40 \\
\hline $\begin{array}{l}\text { Ich gerate oft in Schwierigkeiten, weil ich } \\
\text { etwas tue, ohne zu überlegen. }\end{array}$ & $\begin{array}{l}.28 \\
(.45)\end{array}$ & .59 & $\begin{array}{l}.29 \\
(.46)\end{array}$ & .59 & $\begin{array}{l}.30 \\
(.46)\end{array}$ & .59 \\
\hline $\begin{array}{l}\text { Ich gerate oft in unangenehme Situationen, } \\
\text { weil ich vorher nicht genügend nachge- } \\
\text { dacht habe. }\end{array}$ & $\begin{array}{l}.27 \\
(.44)\end{array}$ & .56 & $\begin{array}{l}.29 \\
(.45)\end{array}$ & .58 & $\begin{array}{l}.26 \\
(.44)\end{array}$ & .57 \\
\hline $\begin{array}{l}\text { Ich gerate oft in Schwierigkeiten, weil ich } \\
\text { mich nicht genügend beherrschen kann. }\end{array}$ & $\begin{array}{l}.22 \\
(.41)\end{array}$ & .43 & $\begin{array}{l}.20 \\
(.40)\end{array}$ & .53 & $\begin{array}{l}.19 \\
(.39)\end{array}$ & .44 \\
\hline$M(S D)$ & \multicolumn{2}{|c|}{$1.10(1.31)$} & \multicolumn{2}{|c|}{$1.21(1.35)$} & \multicolumn{2}{|c|}{$1.19(1.31)$} \\
\hline Cronbachs $\alpha$ & \multicolumn{2}{|c|}{.72} & \multicolumn{2}{|c|}{.74} & \multicolumn{2}{|c|}{.71} \\
\hline
\end{tabular}

Anmerkung. $r_{i t}=$ Trennschärfe (Part-Whole-Korrektur). Skalenwerte 0 (,nein“) und 1 (,ja“).

Aus den folgenden vier der insgesamt 16 Empathieitems des IVE (Stadler et al., 2004a) wurde eine Kurzskala Empathie (Summenscore der dichotomen Antworten) gebildet: „Es bedrückt mich, wenn ich sehe, dass jemand ausgelacht wird“, „Es nimmt mich sehr mit, wenn ich jemanden weinen sehe“, „Ich spüre oft Mitgefühl für Leute, denen es schlechter geht als mir“, „Schüler, die oft gehänselt werden, tun mir leid“. Hohe Werte auf dieser Skala stehen für ein ,,ausgeprägtes Einfühlungsvermögen, starkes Miterleben von Gefühlen und Empfindungen anderer Menschen und Lebewesen“" (Stadler et al., 2004a). Mit einem Cronbachs $\alpha_{4 . \text { Klasse }}=.74$, Cronbachs $\alpha_{5 \text {. Klasse }}=.79$, bzw. Cronbachs $\alpha_{6 . \text { Klasse }}=.74$ ist bei ins- 
gesamt vier Items eine befriedigende interne Konsistenz der Skala gegeben, die sich wiederum geringfügig bis deutlich unter dem Niveau der Ausgangsskala bewegt (die interne Konsistenz lag in der Eichstichprobe zum Vergleich bei Cronbachs $\alpha_{10 \text { Jahre, Jungen }}=.80$, Cronbachs $\alpha_{10 \text { Jahre, Mädchen }}=.66$, Cronbachs $\alpha_{11 \text { Jahre, }}$ Jungen $=.87$, Cronbachs $\alpha_{11 \text { Jahre, Mädchen }}=.81$, Cronbachs $\alpha_{12}$ Jahre, Jungen $=.86$, Cronbachs $\alpha_{12 \text { Jahre, Mädchen }}=.87$; Skalen- und Itemkennwerte siehe Tabelle 24).

Tabelle 24. Skalen- und Itemkennwerte der Skala Empathie nach Messzeitpunkt.

\begin{tabular}{|c|c|c|c|c|c|c|}
\hline & \multicolumn{2}{|c|}{ 4. Klasse } & \multicolumn{2}{|c|}{ 5. Klasse } & \multicolumn{2}{|c|}{ 6. Klasse } \\
\hline & $\begin{array}{c}M \\
(S D)\end{array}$ & $\boldsymbol{r}_{i t}$ & $\begin{array}{c}M \\
(S D)\end{array}$ & $\boldsymbol{r}_{i t}$ & $\begin{array}{c}M \\
(S D)\end{array}$ & $\boldsymbol{r}_{i t}$ \\
\hline $\begin{array}{l}\text { Es bedrückt mich, wenn ich sehe, dass je- } \\
\text { mand ausgelacht wird. }\end{array}$ & $\begin{array}{l}.78 \\
(.41)\end{array}$ & .57 & $\begin{array}{c}.79 \\
(.41)\end{array}$ & .63 & $\begin{array}{l}.77 \\
(.42)\end{array}$ & .55 \\
\hline $\begin{array}{l}\text { Es nimmt mich sehr mit, wenn ich jeman- } \\
\text { den weinen sehe. }\end{array}$ & $\begin{array}{l}.72 \\
(.45)\end{array}$ & .52 & $\begin{array}{l}.74 \\
(.44)\end{array}$ & .55 & $\begin{array}{c}.69 \\
(.47)\end{array}$ & .50 \\
\hline $\begin{array}{l}\text { Ich spüre oft Mitgefühl für Leute, denen es } \\
\text { schlechter geht als mir. }\end{array}$ & $\begin{array}{l}.81 \\
(.39)\end{array}$ & .51 & $\begin{array}{l}.82 \\
(.39)\end{array}$ & .58 & $\begin{array}{l}.79 \\
(.41)\end{array}$ & .52 \\
\hline $\begin{array}{l}\text { Schüler, die oft gehänselt werden, tun mir } \\
\text { leid. }\end{array}$ & $\begin{array}{c}.82 \\
(.38)\end{array}$ & .54 & $\begin{array}{l}.79 \\
(.41)\end{array}$ & .62 & $\begin{array}{l}.77 \\
(.42)\end{array}$ & .57 \\
\hline$M(S D)$ & \multicolumn{2}{|c|}{$3.12(1.23)$} & \multicolumn{2}{|c|}{$3.10(1.30)$} & \multicolumn{2}{|c|}{$2.98(1.30)$} \\
\hline Cronbachs $\alpha$ & \multicolumn{2}{|c|}{.74} & \multicolumn{2}{|c|}{.79} & \multicolumn{2}{|c|}{.74} \\
\hline
\end{tabular}

Anmerkung. $r_{i t}=$ Trennschärfe (Part-Whole-Korrektur). Skalenwerte 0 (,nein“) und 1 (,ja“).

Gleichzeitig hohe Werte auf der Skala Impulsivität und niedrige Werte auf der Skala Empathie können bedeuten, dass „bei gering ausgeprägter Empathie [...] die Folgen impulsiven Verhaltens (z.B. enthemmt-aggressive Verhaltenstendenzen) im sozialen Kontext gravierender sein [können und] unter ungünstigen Bedingungen erhöhtes Risiko für die Entwicklung antisozialer Verhaltenstendenzen“ (Stadler, Janke \& Schmeck, 2004b, S. 23) bestehen kann.

SDQ-Deu

00045

Im Fragebogen der fünften und sechsten Klasse wurden die drei Konstrukte „Hyperaktivität“, „Verhaltensprobleme mit Gleichaltrigen“ und ,prosoziales Verhalten" mithilfe des Strengths and Difficulties Questionnaire in der deutschen Übersetzung (SDQ-Deu; Goodman, 1997; Klasen et al., 2000), einem Screeningfragebogen ,zur Erfassung von Verhaltensstärken und -auffälligkeiten bei Kindern und Jugendlichen im Alter von 4 bis 16 Jahren“ (Klasen, Woerner, Rothenberger \& Goodman, 2003, S. 492), erhoben. 
Hyperaktivität wird in diesem Instrument über die folgenden vier dreistufigen (,nicht zutreffend“ - 0, „teilweise zutreffend“ - 1, „eindeutig zutreffend“ - 2) Items erfasst: „Ich bin oft unruhig; Ich kann nicht still sitzen“ (0-1-2), „Ich bin dauernd in Bewegung oder zappelig“ (0-1-2), „Ich lasse mich leicht ablenken; Ich finde es schwer, mich zu konzentrieren“ (0-1-2), „Ich denke nach, bevor ich handle“ (2-1-0), „Was ich angefangen habe, mache ich zu Ende; Ich kann mich lange genug konzentrieren“ (2-1-0). Summenwerte zwischen null und fünf stehen für ein normales, Summenwerte von sechs für ein grenzwertiges sowie Summenwerte von sieben bis zehn für auffälliges Verhalten (vgl. Goodman, 2005). ${ }^{40}$ Mit einem Cronbachs $\alpha_{5 \text {. Klasse }}=.61$, bzw. Cronbachs $\alpha_{6 . \text { Klasse }}=.62$ konnte im Berliner Längsschnitt Medien ein zu anderen Studien vergleichbares bzw. akzeptables $\mathrm{Ma} \beta$ der internen Konsistenz erzielt werden (England: Cronbachs $\alpha=.67$; Goodman, 2001; Niederlande: Cronbachs $\alpha=.66$; Widenfelt, Goedhart, Treffers \& Goodman, 2003).

Die Erfassung der Verhaltensprobleme mit Gleichaltrigen weist eine große Nähe zum Konstrukt soziale Integration des FEESS (Rauer \& Schuck, 2003) auf; folgende Items werden eingesetzt: „Ich bin meistens für mich alleine; ich beschäftige mich lieber mit mir selbst" (,nicht zutreffend“- 0, „teilweise zutreffend“ - 1 , ,eindeutig zutreffend“ - 2), ,Ich habe einen oder mehrere gute Freunde oder Freundinnen“ (2-1-0), „Im Allgemeinen bin ich bei Gleichaltrigen beliebt“ (2-1-0), „Ich werde von anderen gehänselt oder schikaniert“ (0-1-2), „Ich komme mit Erwachsenen besser aus als mit Gleichaltrigen“ (0-1-2). Summenwerte zwischen null und drei stehen auf dieser Skala für ein normales, Summenwerte von vier und fünf für ein grenzwertiges sowie Summenwerte von sechs bis zehn für auffälliges Verhalten (vgl. Goodman, 2005). Mit einem Cronbachs $\alpha_{5}$. Klasse $=.42$, bzw. Cronbachs $\alpha_{6 . \text { Klasse }}=.56$ konnte ein im Vergleich zu anderen Studien vergleichbares bzw. besseres $\mathrm{Ma} ß$ der internen Konsistenz erzielt werden (England: Cronbachs $\alpha=.41$; Goodman, 2001; Niederlande: Cronbachs $\alpha=$. 39; Widenfelt et al., 2003). Trotz dieser immer noch schlechten Kennwerte wurde aus Gründen der Vergleichbarkeit mit den anderen beiden Skalen des SDQ auch diese Skala in die Auswertungen mit aufgenommen.

Die Skala prosoziales Verhalten umfasst die folgenden Items (,nicht zutreffend“- 0 , „teilweise zutreffend“ - 1 , „eindeutig zutreffend“ - 2): „Ich versuche, nett zu anderen Menschen zu sein, ihre Gefühle sind mir wichtig“, „Ich teile normalerweise mit Anderen (z. B. Süßigkeiten, Spielzeug, Buntstifte)“, „Ich bin hilfsbereit, wenn andere verletzt, krank oder traurig sind“, „Ich bin nett zu jüngeren Kindern“, „Ich helfe anderen oft freiwillig (Eltern, Lehrer, Gleichaltrige)“.

40 Diese Einteilung wurde verteilungsbasiert vorgenommen, so dass $80 \%$ der Kinder als normal, $10 \%$ als grenzwertig auffällig und $10 \%$ als auffällig eingestuft wurden (vgl. Goodman, 2005). 
Summenwerte zwischen sechs und zehn stehen auf dieser Skala für ein normales, Summenwerte von fünf für ein grenzwertiges sowie Summenwerte von null bis vier für auffälliges Verhalten (vgl. Goodman, 2005). Mit einem Cronbachs $\alpha_{5}$. Klasse $=.74$, bzw. Cronbachs $\alpha_{6 . \text { Klasse }}=.77$ konnte im Berliner Längsschnitt Medien wiederum ein im Vergleich zu anderen Studien besseres und insgesamt akzeptables $\mathrm{Ma} ß$ der internen Konsistenz erzielt werden (England: Cronbachs $\alpha=.66$; Goodman, 2001; Niederlande: Cronbachs $\alpha=.60$; Widenfelt et al., 2003).

\subsubsection{Instrumente und Skalen des KFN-Elternfragebogens}

\section{Lieblingsaktivitäten der Kinder}

In den Elternfragebögen zum Halbjahr der dritten Klasse und am Ende der vierten Klasse wurde in einem offenen Antwortformat das Freizeitverhalten (vgl. Abbildung 18) sowie das Sozialverhalten der Schülerinnen und Schüler, unter anderem in den Bereichen Sportarten, Hobbys, Vereinsmitgliedschaften und Pflichten zu Hause (Elternfragebogen über das Verhalten von Kindern und Jugendlichen - CBCL/4-18, Fragen I-VI; Arbeitsgruppe Deutsche Child Behavior Checklist, 1998).

\begin{tabular}{|l|l} 
20. Nennen Sie bitte die Lieblingsaktivitäten, Hobbies oder Spiele Ihres Kindes (z. B. Singen, \\
Klavierspielen, Lesen, mit Puppen oder Autos spielen usw., außer Sport, Radiohören, Fernsehen, \\
Computerspielen).
\end{tabular}

Abbildung 18. Erhebung der Lieblingsaktivitäten der Kinder im Elternfragebogen.

Familiäre Mediennutzungsmuster

100345

Zur Bestimmung eines familiären „Mediennutzungsklimas“ wurde zu den unterschiedlichen Messzeitpunkten in den Elternfragebögen in einem offenen Antwortformat u. a. die Nutzungszeiten für Fernsehen, Computerspielen und Internetnutzung (nur fünfte und sechste Klasse) getrennt für den oder die Beantwortende(n) selbst, das Kind welches an der Befragung teilgenommen hat sowie den 
Partner/die Partnerin (nur fünfte und sechste Klasse) für einen Wochentag und einen Samstag erhoben (siehe Abbildung 19).

\begin{tabular}{|c|c|c|c|}
\hline \multicolumn{4}{|c|}{$\begin{array}{l}\text { 28. Wie lange spiele Ihr Kind, Sie bzw. Ihr Partner im Durchschnitt an einem Samstag Computer- } \\
\text { oder Videospiele? (Bitte Antwort eintragen.) }\end{array}$} \\
\hline mein Kind & Stunde(n) und & Minuten & $\square$ Mein Kind spielt keine Computerspiele \\
\hline Ich & Stunde(n) und & Minuten & $\square$ Ich spiele keine Computerspiele \\
\hline Mein Partner & ca. __ Stunde $(n)$ und & Minuten & $\square$ Mein Partner spielt keine Computerspiele \\
\hline
\end{tabular}

Abbildung 19. Erhebung der Medienzeiten im Elternfragebogen am Beispiel des Computerspielens an einem Samstag.

\section{Medienerziehung}

100345

Analog zu den Kinderfragebögen wurde auch in den Elternfragebögen der unterschiedlichen Messzeitpunkte die durch die Eltern wahrgenommene Medienerziehung ihrer Kinder für die Bereiche Fernsehen, Computerspiele und Internet erhoben. Wie auch für die Kinderangaben wurde für die Elternangaben eine Skala regulativen Monitorings getrennt für die unterschiedlichen Bildschirmmedien gebildet. $^{41}$

Tabelle 25. Skalen- und Itemkennwerte der Skala regulatives Monitoring nach Bildschirmmedium und Messzeitpunkt (Elternangaben).

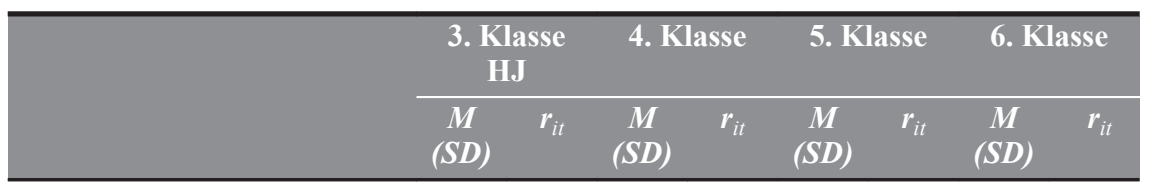

\section{Fernsehnutzung}

Bei uns gibt es klare Regelungen was mein Kind sehen darf und was nicht.

$\begin{array}{llll}3.39 & .48 & 3.32 & .50 \\ (.74) & .46) & (.76\end{array}$

Ich will wissen was sich mein Kind im Fernsehen anschaut. Wir haben klare zeitliche Regelungen.

\begin{tabular}{llll}
3.70 & .38 & 3.69 & \\
$(.56)$ & .41 & $(.54)$ & .41 \\
2.90 & & 2.98 & \\
$(.94)$ & .38 & $(.95)$ & .56 \\
\hline 9.95 & $(1.72)$ & 9.84 & $(1.75)$
\end{tabular}

$$
\text { M (SD) } 9.95(1.72) \quad 9.84(1.75)
$$

$\begin{array}{llll}\text { Cronbachs } \alpha & .59 & .62\end{array}$

41 Summenvariable. Zur theoretischen Einordnung der Skala siehe Kapitel 1.5.1 Medienerziehung. 


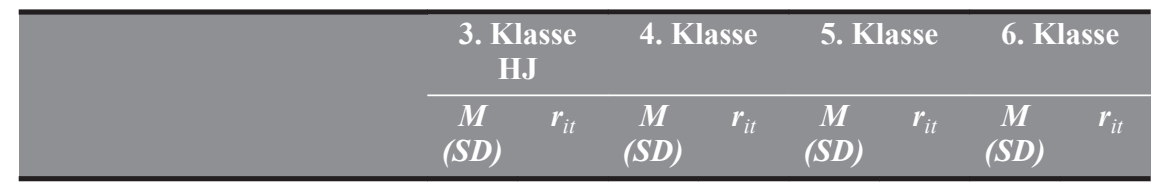

\section{Computerspielnutzung}

Bei uns gibt es klare Regelungen welche Spiele mein Kind spielen darf und welche nicht.

\begin{tabular}{|c|c|c|c|c|c|c|c|}
\hline $\begin{array}{l}3.79 \\
(.58)\end{array}$ & .40 & $\begin{array}{l}3.76 \\
(.53)\end{array}$ & .44 & $\begin{array}{l}3.63 \\
(.69)\end{array}$ & .58 & $\begin{array}{l}3.53 \\
(.68)\end{array}$ & .53 \\
\hline $\begin{array}{l}3.69 \\
(.73)\end{array}$ & .53 & $\begin{array}{l}3.70 \\
(.68)\end{array}$ & .48 & $\begin{array}{l}3.63 \\
(.78)\end{array}$ & .68 & $\begin{array}{l}3.61 \\
(.74)\end{array}$ & .64 \\
\hline $\begin{array}{l}3.16 \\
(.10)\end{array}$ & .34 & $\begin{array}{l}3.17 \\
(.97)\end{array}$ & .38 & $\begin{array}{l}3.29 \\
(.97)\end{array}$ & .50 & $\begin{array}{l}3.12 \\
(.98)\end{array}$ & .47 \\
\hline 10.5 & $.85)$ & 10.57 & $1.70)$ & 10.5 & 2.01) & 10.2 & .93 \\
\hline
\end{tabular}

Ich weiß welche Spiele mein Kind spielt.

Wir haben klare zeitliche Regelungen.

\section{$M(S D)$}

.59

.59

.74

\section{Internetnutzung}

Bei uns gibt es klare Regelungen was mein Kind im Internet machen darf und was nicht.

Ich weiß was mein Kind im Internet macht.

$\begin{array}{llll}3.68 & .57 & 3.61 & .56 \\ (.77) & & (.72) & \end{array}$

Wir haben klare zeitliche Regelungen.

Anmerkung. $r_{i t}=$ Trennschärfe (Part-Whole-Korrektur). Skalenwerte von 1 bis 4 (,nie“, „,manchmal" „meistens“, „immer“).

Mit einem Cronbachs $\alpha$ von $\alpha_{3 . \text { Klasse HJ }}=.59$ und $\alpha_{6 . \text { Klasse }}=.62$ für die Fernsehnutzung, zwischen $\alpha_{3 . \text { Klasse } H J}=.59$ und $\alpha_{6 . \text { Klasse }}=.71$ für die Computerspielnutzung sowie einem Cronbachs $\alpha_{5 . \text { Klasse }}=.70$ und Cronbachs $\alpha_{6 . \text { Klasse }}=.66$ für die Internetnutzung ist bei insgesamt jeweils drei Items eine (gerade noch) befriedigende interne Konsistenz der Skala gegeben, die im Vergleich zu den Kinderangaben deutlich geringer ausfällt (Skalen- und Itemkennwerte siehe Tabelle 25). Aus Gründen der Vergleichbarkeit der Kinder- und Elternskalen wurden jedoch keine Skalenveränderungen durchgeführt.

Die Skala regulatives Monitoring (Elternangaben) wurde analog zur Skala der Kinder für die unterschiedlichen Bildschirmmedien nach der folgenden Vorschrift umcodiert: 3-4 Punkte $=$ kein regulatives Monitoring; 5-7 Punkte $=$ gerin- 
ges regulatives Monitoring; 8-10 Punkte $=$ mittleres regulatives Monitoring; 11-12 Punkte $=$ hohes regulatives Monitoring. ${ }^{42}$

Technische Hilfsmittel - Internetnutzung der Kinder

00045

In den Elternfragebögen der fünften und sechsten Klasse wurde zusätzlich zu den Angaben zur elterlichen Medienerziehung erhoben, welche technischen Möglichkeiten von den Eltern genutzt werden, um den Internetkonsum ihrer Kinder sowohl zeitlich als auch inhaltlich kontrollieren zu können (siehe Abbildung 20).

\begin{tabular}{|l|l|}
\hline $\begin{array}{l}\text { 20. Welche der folgenden Hilfsmittel sind auf dem Computer installiert, mit dem Ihr Kind in der Regel ins } \\
\text { Internet geht? (Bitte kreuzen Sie in jeder Zeile das Zutreffende an.) }\end{array}$ \\
\hline \multicolumn{2}{|c|}{ Ja } \\
\hline Filtersoftware & \\
\hline Kindersicherung (etwa durch Passwort) & $\square$ \\
\hline Kinderbrowser & $\square$ \\
\hline Kindersuchmaschine & $\square$ \\
\hline Zeitschaltuhr (Software oder Hardware) & $\square$ \\
\hline
\end{tabular}

Abbildung 20. Erhebung der genutzten technischen Hilfsmittel bei der kindlichen Internetnutzung.

\section{Jugendmedienschutz}

In den Elternfragebögen der sechsten Klasse wurde darüber hinaus die Einschätzung der Eltern zur bestehenden Praxis des Jugendmedienschutzes im Bereich der Computerspiele mit den Fragen aus Abbildung 21 erhoben.

42 Eltern mit keinem regulativen Monitoring konnten somit maximal nur einem Item mit „manchmal“ zustimmen. Eltern mit hohem regulativem Monitoring haben auf der anderen Seite mindestens zwei Items mit ,immer“ zugestimmt. Entsprechend haben Eltern mit geringem regulativem Monitoring im Mittel $( \pm 1)$ mit ,,manchmal“ und Eltern mit mittlerem regulativen Monitoring im Mittel $( \pm 1)$ mit ,meistens“ zugestimmt. 
Der Jugendmedienschutz in Deutschland ist in den letzten Jahren mehrfach reformiert worden. So ist seit einigen Jahren auf jedem in Deutschland verkauften Computerspiel zu lesen, ab welchem Alter dieses Spiel freigegeben ist. Ist ein Spiel beispielsweise "ab 16 Jahren" freigegeben, darf es nicht an jüngere Kinder und Jugendliche verkauft oder verliehen werden. Verstoßen Händler oder Verleiher gegen die Auflagen, drohen Ihnen Geldstrafen. Um zu überprüfen, ob diese Vorgaben auch eingehalten werden, werden seit Kurzem in einigen Regionen besonders geschulte jugendliche Testkäufer eingesetzt, die bewusst versuchen, Spiele zu kaufen, für die sie noch nicht alt genug sind. Gleichzeitig gibt es in der Öffentlichkeit immer wieder Diskussionen darüber, ob die Altersfreigaben für Computerspiele in Deutschland zu streng oder zu lasch sind.

13. Inwieweit stimmen Sie den folgenden Aussagen zum Jugendmedienschutz in Deutschland zu? stimmt nicht stimmt kaum stimmt eher stimmt genau

Die Altersfreigaben für Computerspiele in

Deutschland sollten insgesamt deutlich strenger sein.

Die Altersfreigaben für Computerspiele in Deutschland sind insgesamt viel zu streng.

Testkäufe sind ein gutes Instrument, um den Jugendmedienschutz in Deutschland zu verbessern.

Altersfreigaben für Computerspiele sind ein gutes Instrument, Kindern und Jugendlichen den Zugang zu problematischen Computerspielen zu verwehren.

\section{Abbildung 21. Erhebung der Einschätzung der bestehenden Praxis des Jugendmedienschutzes.}

